\title{
WestVirginiaUniversity
}

THE RESEARCH REPOSITORY @ WVU

Graduate Theses, Dissertations, and Problem Reports

2011

\section{Agent Based Load Management System for a Smart Power Distribution System}

\author{
Manasaveena Chennuri \\ West Virginia University
}

Follow this and additional works at: https://researchrepository.wvu.edu/etd

\section{Recommended Citation}

Chennuri, Manasaveena, "Agent Based Load Management System for a Smart Power Distribution System" (2011). Graduate Theses, Dissertations, and Problem Reports. 3310.

https://researchrepository.wvu.edu/etd/3310

This Thesis is protected by copyright and/or related rights. It has been brought to you by the The Research Repository @ WVU with permission from the rights-holder(s). You are free to use this Thesis in any way that is permitted by the copyright and related rights legislation that applies to your use. For other uses you must obtain permission from the rights-holder(s) directly, unless additional rights are indicated by a Creative Commons license in the record and/ or on the work itself. This Thesis has been accepted for inclusion in WVU Graduate Theses, Dissertations, and Problem Reports collection by an authorized administrator of The Research Repository @ WVU. For more information, please contact researchrepository@mail.wvu.edu. 


\title{
Agent Based Load Management System for a Smart Power Distribution System
}

Manasaveena Chennuri

Thesis submitted to the

\section{College of Engineering and Mineral Resources}

At West Virginia University

In partial fulfillment of the requirements

for the degree of

\author{
Master of Science \\ in \\ Electrical Engineering
}

\author{
Dr Ali Feliachi \\ Dr Muhammad A. Choudhry \\ Dr Sarika K. Solanki
}

\section{Lane Department of Computer Science and Electrical Engineering \\ Morgantown, West Virginia \\ 2011}

Keywords: Multi-Agent Systems, JADE, Peak Demand, Load Forecast 


\title{
ABSTRACT \\ Agent Based Load Management System for a Smart Power Distribution
}

\author{
System
}

\section{Manasaveena Chennuri}

Inherently, electric power system is exceptionally complex as far its planning, construction and operation is concerned. Adding to that, advancement in the way the electric power is consumed and ascending electrical power demand makes the traditional electric power system's planning, monitoring and control an uphill struggle. This thesis work is focused on the improvement of power distribution system. Improvement in the control system, information system, and management system of power distribution system is transforming the grounds on which the most of the traditional power system has been operated.

The present thesis work has been motivated to be a part of an attempt to make the electric power grid smarter. Smart Grid initiative by various power industrial units around the globe is a revolutionary attempt to impart intelligence and robust technology into the existing electric grid to make it highly reliable with effective capacity utilization. This thesis work presents a novel approach to accommodate distributed generation resources in the power distribution system helping to reduce the peak power demand by 15 percent.

Demand dispatch method which is a novel approach to demand response is implemented in this thesis. Demand dispatch is the capability to aggregate and precisely control individual loads on command. The dispatch algorithm makes use of controllable loads which can be turned on and off with unnoticeable interruption. The load is forecasted and it is dispatched accordingly using distributed generation resources and controllable loads there-by helping to reduce peak demand.

Multi-agent system is adopted to manage the demand dispatch simulation. Multi-agents are collection of agents which are capable of perceiving the environment in which they are located and act on it by communicating with each other to achieve the goals. Load is forecasted in MATLAB and multi-agents programmed in JADE utilize the forecasted load data to dispatch the load in such a way so as to reduce the peak demand. Agents are located at demand aggregator level, zone level and DG level. These agents communicate and negotiate to dispatch the load appropriately based on resources and load availability. 


\section{DEDICATION}

I would like to dedicate this thesis to my mom, who is my inspiration for any small success in my life. 


\section{ACKNOWLEDGMENT}

I wish to express my deep sense of gratitude and indebtedness to my academic advisors, Dr. Ali Feliachi for his great support in the course of this research and opportunity to broaden my horizons. I also wish to express my sincere appreciation to Dr. Muhammad A. Choudhry and Dr. Sarika K. Solanki for being in my advisory committee.

I am much obliged for the assistance provided by the APERC (Advanced Power and Electricity Research Center) for supporting me as a graduate research assistant. I owe my deepest gratitude to my friend Uday for his help in Jade programming. I would like to thank my research mates Shola, Rabie, Naveen and Vaidhyanath for their quick and sharp comments for my thesis progress and warm work environment.

I owe my heartfelt regards to my mom, dad, sister and brother who have been my constant source of inspiration, motivation and encouragement. My special thanks to Sunny, Vamsi, Kedar, KC, Soumya and Srikanth for all the support. 


\section{Contents}

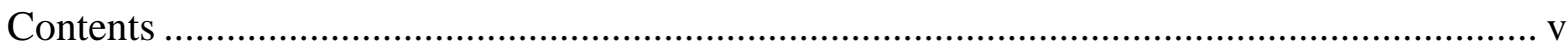

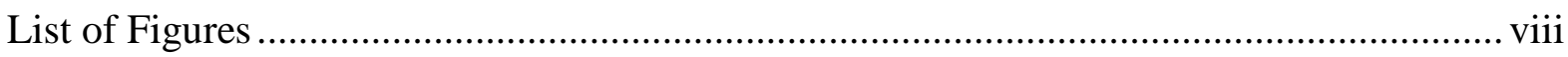

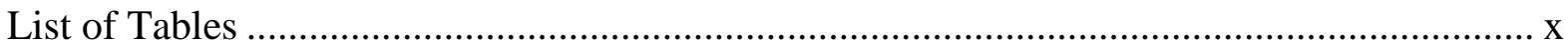

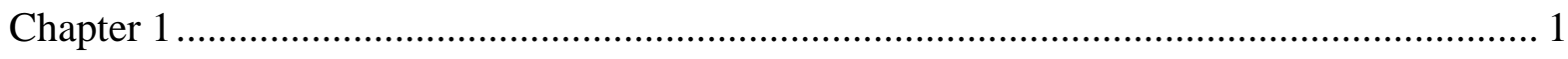

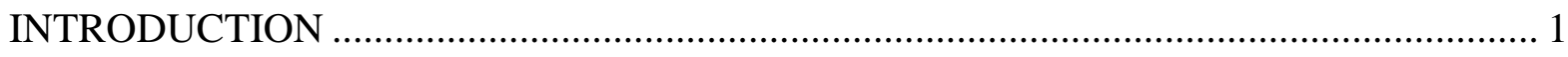

1.1 Background ................................................................................................. 1

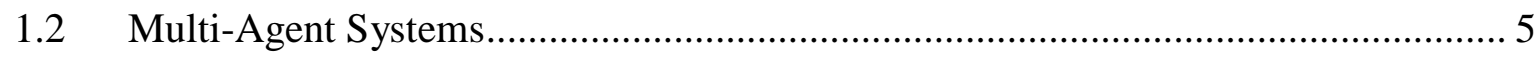

1.2.1 Agent Communication and Coordination ........................................... 8

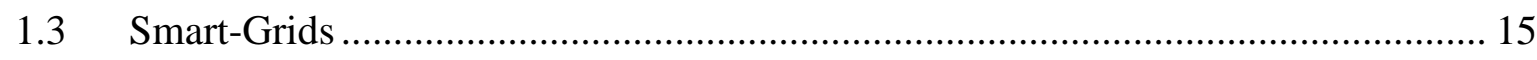

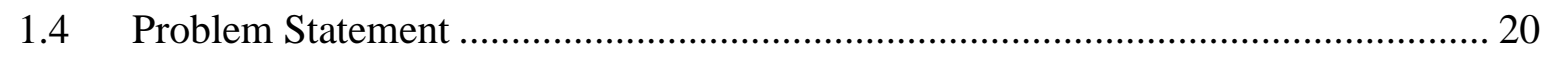

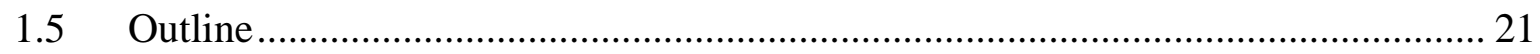

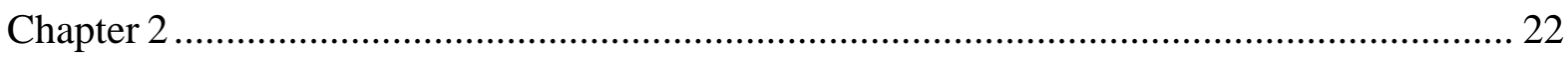

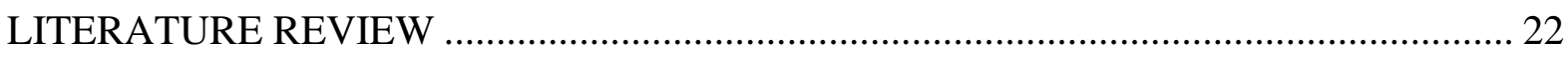

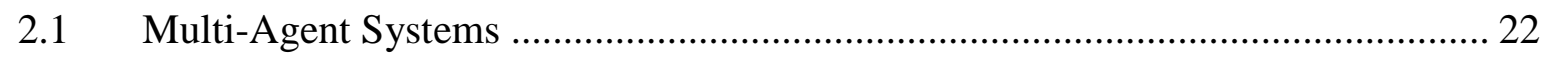

2.1.1 Multi-Agent Systems Applications Overview ....................................... 22

2.1.2 Multi-Agent Systems in Smart Grids................................................. 25

2.1.3 Multi-Agent Systems in Demand Management........................................ 28

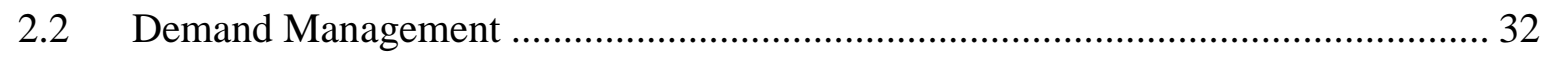


2.3 Challenges for Peak Demand Reduction......................................................... 37

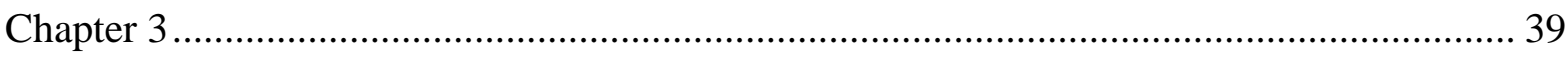

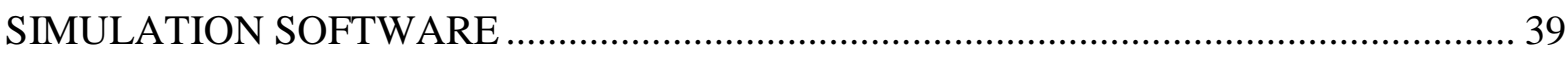

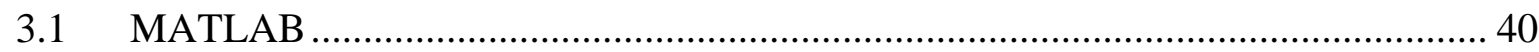

3.2 Java Agent DEvelopment framework ........................................................... 42

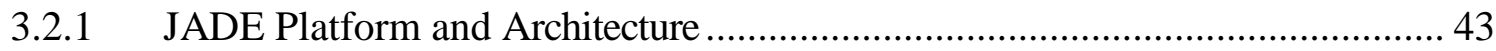

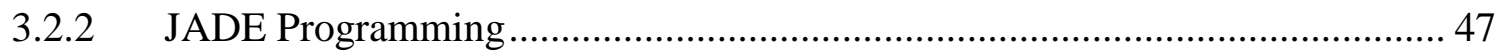

3.3 IBM ILOG CPLEX Optimization Studio................................................ 48

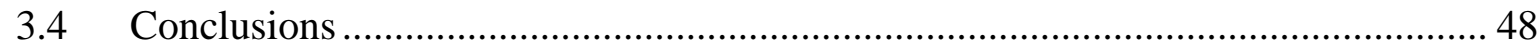

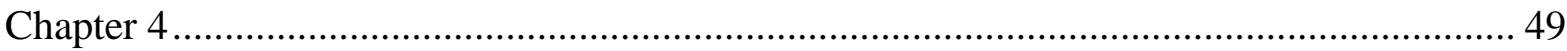

SIMULATIONS AND RESULTS _.......................................................................... 49

4.1 Load Forecaster ....................................................................................... 49

4.1.1 Database for Load Forecasting ........................................................... 49

4.1.2 Linear regression............................................................................ 51

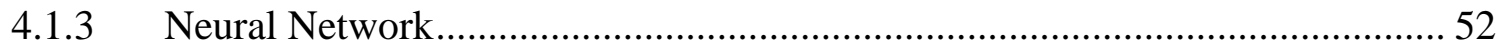

4.1.4 Load Forecasting with Neural Network Prediction .................................... 57

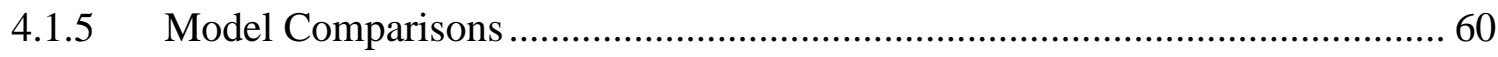

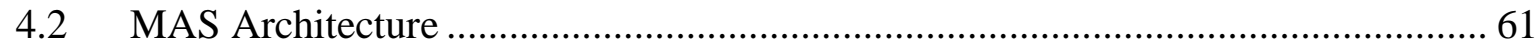

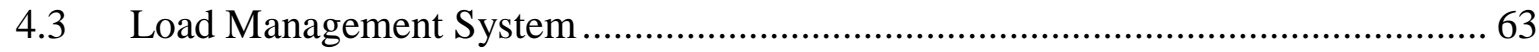

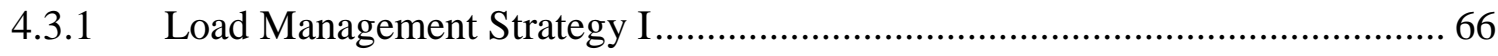




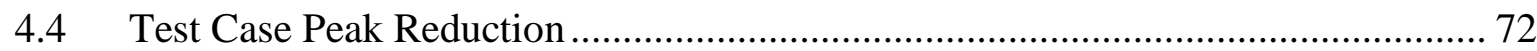

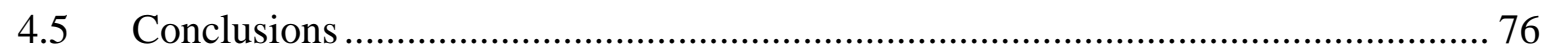

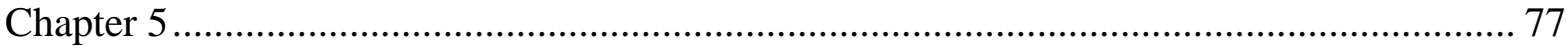

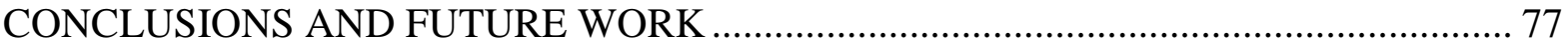

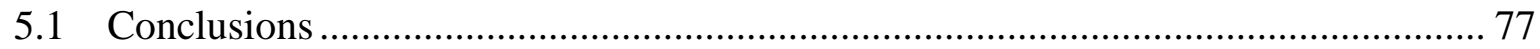

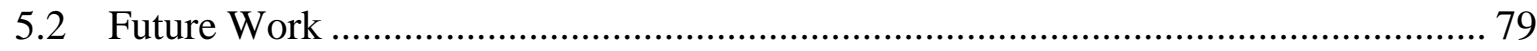

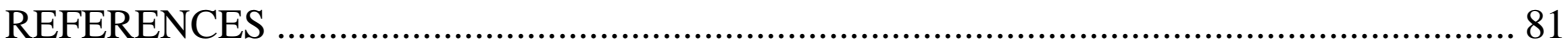




\section{List of Figures}

Figure 1- 1: A taxonomy of some of the different ways in which agents can coordinate their

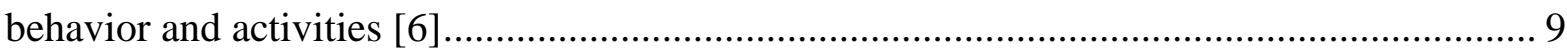

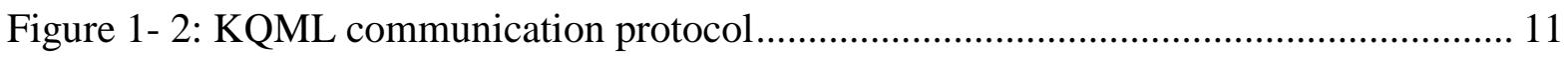

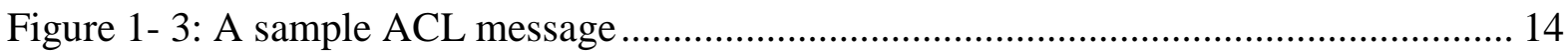

Figure 2- 1: System flow chart for MAS based CAPP for manufacturing [23] ................... 23

Figure 2- 2: Communication flow in MAS for smart grid simulation [29] ......................... 26

Figure 2- 3: DER integration plan for advanced distribution operation ........................... 28

Figure 2- 4: Future anticipated electric network 2040 [33] ......................................... 30

Figure 2- 5: PowerMatcher hierarchy based architecture [34] ......................................... 31

Figure 2- 6: Two load curves, showing the historical peak of $4400 \mathrm{MW}$ on $7^{\text {th }}$ January, 2003

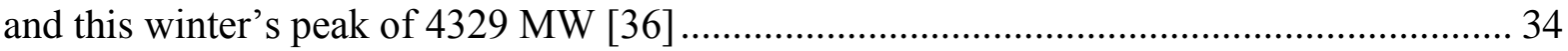

Figure 2- 7: Block diagram of dispatchable PV arrays using storage [38] ........................ 35

Figure 3- 1: Simulation Model Architecture.................................................................. 40

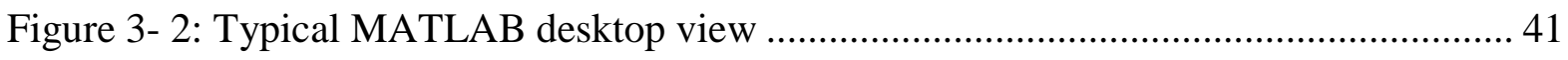

Figure 3- 3: Reference architecture of a JADE agent [43] .......................................... 45

Figure 3- 4: Main architectural elements and relationship between them [4] ..................... 46

Figure 4- 1: Visual query builder interface from Database toolbox ................................. 50

Figure 4- 2: Prediction of accuracy with linear regression ........................................... 52

Figure 4- 3: Neural network training block diagram ................................................ 53

Figure 4- 4: Neural network layers with number of neurons ........................................ 54

Figure 4- 5: System load forecast results using neural network toolbox ............................ 55

Figure 4- 6: Neural network regression plot during training ....................................... 55 
Figure 4- 7: Neural network regression plot during validation............................................ 56

Figure 4- 8: Neural network regression plot during validation........................................... 56

Figure 4- 9: Snapshot of neural networks training tool interface .........................................57

Figure 4- 10: Load Forecast Predicted by Neural Network Model and Actual Load............. 59

Figure 4- 11: Load Forecast Predicted by Neural Network Model and Actual Load.............. 60

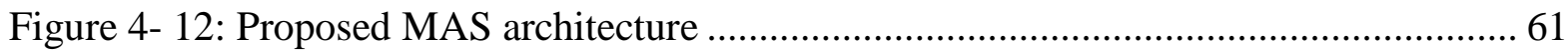

Figure 4- 13: Depiction of agent's location on West Run Feeder .......................................... 63

Figure 4- 14: Load Forecasting Flowchart............................................................................... 64

Figure 4- 15: MAS action sequences for peak load reduction by 15 percent in strategy I..... 67

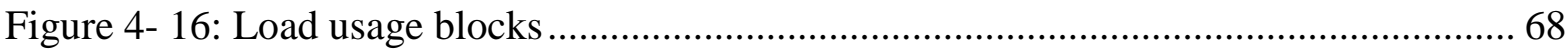

Figure 4- 17: Load profile with possible MW availability ...................................................... 68

Figure 4- 18: MAS action sequences for peak load reduction by 15 percent in strategy II.... 71

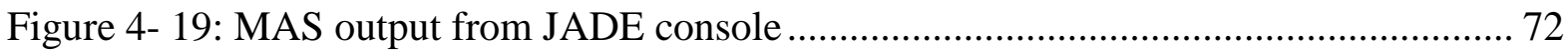

Figure 4- 20: Actual, forecasted and peak managed system load curves .............................. 75 


\section{List of Tables}

Table 1- 1: FIPA ACL language parameters and their interpretation ................................ 12

Table 1- 2: Gap and proposed smart grid solution [44] ................................................. 18

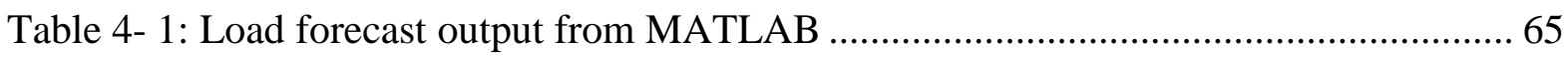

Table 4- 2: (a) Load Management Agent (LMA) Data, (b) Zone Agent (ZA) Data, (c)

Distributed Generation Agent (DGA), and (d) Load Aggregator Agent (LAA) data ........... 66 
NOMENCLATURE

$\begin{array}{ll}\text { ACL } & \text { Agent Communication Language } \\ \text { DER } & \text { Distributed Energy Resources } \\ \text { DG } & \text { Distributed Generators } \\ \text { FIPA } & \text { Foundation for Intelligent Physical Agents } \\ \text { GUI } & \text { Graphical User Interface } \\ \text { JADE } & \text { Java Agent Development Framework } \\ \text { KQML } & \text { Knowledge Query and Manipulation Language } \\ \text { MAS } & \text { Multi-agent System } \\ \text { OOP } & \text { Object Oriented Programming } \\ \text { WVU } & \text { West Virginia University }\end{array}$




\section{Chapter 1}

\section{INTRODUCTION}

\subsection{Background}

The major role of the electric power system is to supply power to the end customer through highly complex power grid comprising of substations, lines and devices at various power levels and voltage ranges. It also has to deal with the management of highly volatile power generation, power demand and power markets. Right from the early ages of discovery of electromagnetic phenomenon, which is still the basic principle for today's power generation, there was always been an attempt to generate it with more adaptability. Generation, transmission and distribution are the three very important processes in supplying the power to the consumer.

Initially, the power used was mainly generated from hydro-electric power generation stations and thermal (coal) power generation station. As the technology manifested and also the awareness for the environmental friendly activities increased, the power generation is following various trends. Renewable energy resources like bio-fuel, bio-mass, geothermal, solar energy, tidal power, wave power and wind power have been real area of interests for the power generation. However, the availability of these resources and integration of them to the actual power grid still stands as a complex issue. 
Apart from separating and operating the generation and transmission of electric power as two different processes, the power transmission system did not really see major trends as compared to the power distribution system. With increase in the urbanization, industrial consumption and many other factors, the power demand is constantly ascending and so is its complexity. As known, traditionally power is transmitted and distributed from central power generating station through transmission substations, transmission lines, distribution sub-stations and distribution lines. Planning and construction of all these is inherently expensive and complex. With the change in the power consumption and demand there comes the necessity for the expansion of the prior mentioned power units which is very pricey. The alternative and eco-friendly option is to install small range renewable power generating units near the consumer location integrating them to the distribution grid. These small range power generating units are generally called Distributed Generators (DGs) or Distributed Energy Resources (DERs) which storage. Introducing DGs to the power grid is to make power systems more reliable and secure. Apart from the above mentioned benefits of the DGs, they are 60 to 80 percent efficient where as traditional generating units are 35 percent efficient [1]. As a complement to traditional electric power system, DGs could lower the overall cost of the producing and delivering electric power, promote the development and wider use of renewable energy. It also helps in decreasing the congestion on power lines resulting in less brownouts and blackouts.

Operating the power grid in normal condition is itself a great deal and managing peak demand needs a lot of effort. Peak demand is that period of a day/month/year where the electrical power is expected to be provided significantly higher than average supply level. Various predictive methods are adopted to forecast the peak demand so that available resources can be economically 
managed to supply the demand. Peak demand can occur during hours in a day, days in month and months in a year. Various factors affect the peak demand like the weather condition, seasons of the year, working days or holidays, time of the day etc.

The major concern with the peak demand is to supply it. Though there is a lot of peak demand forecasting methods practiced, it still remains uncertain that when and how much it is going to peak. Now-a-days when the power grid is integrated with intermittent renewable energy resources this problem of operating the grid with stability becomes tougher. Just to supply the peak demand few power plants are maintained which can be fired up very fast like natural gas power generating units and they are called peaking power plants. These power plants are used mostly as back up generations or during peak demand time and still requires continuous maintenance making the energy price during the peak demand time more expensive than the usual kilowatt-hour charge. Also to encourage the power usage during off-peak hours which results in the reduction of peak demand few utilities charge lower price per kilowatt-hour during off-peak periods. So, the peak demand results in reducing the overall cost of the electricity usage.

Many attempts are made and a lot of research is still undergoing to study the methods for the reduction of peak demand. Various initiatives are taken by different utilities like providing credits to customers who reduce their usage during peak demand period. Load management techniques like peak shifting, peak shaving, peak sharing, etc. are adopted to reduce the peak. Independent System Operators (ISO) or utilities sign the customers for participation during peak demand period, helping them to control the load to manage the peak demand. Integration of DGs 
into the grid can also help in reducing the peak demand, by supplying the local load and reducing the congestion on power lines, thereby reducing the peak demand.

Peak load management strategies are basically robust in nature. Not all utilities have different tariffs for customers during peak demand time. This makes the problem tougher to handle because involvement of the customers decreases, thereby it becomes harder to manage the load. Demand response has been an area of interest since decades. Demand response is simply the customer response in terms of electric usage to the changes in electricity generation. Demand response can also be the curtailment of load or generating power at local unit which may or may not be connected in parallel to the grid [2].

Traditionally load shedding is the considerable option for decreasing the peak demand but lately, in most of the areas, load shedding and prioritizing the loads is forbidden. In such cases the better option is to study the loads and control them in such a way that their usage can be monitored and altered. Apart from customers having the control on their loads, utilities or ISOs are given control on the loads in a way helping them to turn On the loads during Off peaks and turning loads Off during On peak without noticeable interruption of service. Introducing advance energy management systems and devices would help to achieve the task. Novel technique called Multi-agent systems can be effectively integrated to the grid to impart intelligence so that the monitoring and control of the load can be economically achieved. 


\subsection{Multi-Agent Systems}

Multi-agents systems are those systems that include multi autonomous entities (software or hardware) with either diverging information or diverging interest or both [3]. A set of software or hardware entities trying to communicate to achieve the task assigned on our behalf with best possible option forms a multi-agent system. In order to be successful the agents need to embody our preferences and our knowledge about similar agents with which they interact and also about the environment in which they will be operated [3].

Multi-agent system technology has been a subject of extensive discussion and investigation within the research community since several years, but in recent years it has seen significant degree of exploitation in marketable applications [4]. The demand for multi-agent technology is because of its versatile applications. These multi-agent systems are being used in an increasingly wide variety of applications, ranging from relatively small systems for personal assistance to intricate applications for industrial applications [4]. Before discussing further about multi-agent system's architecture, programming languages, and tools we will get little more into single agent and its attributes.

The term 'agent' has been used in a number of technologies like in applied artificial intelligence, databases, operating systems and computer networking literature [4]. So as many technical terms, the term 'agent' is defined differently. But all those definitions agree that an agent is essentially an exceptional software module that has autonomy that provides an interoperable interface to an arbitrary system and/or like a human agent, working for some clients in quest of 
its agenda [4]. As described by Michael Wooldridge and Nicholas R. Jennings an agent has attributes as autonomy, social ability, reactivity and pro-activeness [intelligent agents]. In detail:

- Autonomy : without the direct intervention of human or other agents can be operated and have control over their action and internal state (Castelfranchi, 1995);

- Social-ability : agents will be interacting with other agents and if needed with humans using agent communication language (Geneserth and Ketchpel, 1994);

- Reactivity : agents perceive their environment and respond in timely fashion to the changes that occur in it;

- Pro-activeness: Agents take the initiative and act in response to their environment in goal-oriented direction.

Various other attributes like veracity, benevolence, mobility, rationality and emotions (knowledge, belief, intension and obligations) are considered to be owned by agents along with the above mentioned attributes [7]. These concepts are actually implemented using concepts that are more usually applied to humans. However, this thesis assumes all the basic attributes of the agent. As mentioned earlier when all these single agents communicate and negotiate to come up with a decision and solve the assigned task they form a multi-agent system. The critical process for the agent is to formulate a decision to make right action in order to make task successful. It depends on the environment in which the agents are operated. As proposed by Russell and Norvig [8] following are the classification of environments in which agents can be possibly operated:

- Accessible vs. Inaccessible: Accessible environments are those in which all states can be readily accessible by agents and is not possible in inaccessible; 
- Deterministic vs. Non-Deterministic: When effects of the actions on the environment can be anticipated then the environment can be categorized as deterministic and non-deterministic makes it complex to interpret the effects;

- Episodic vs. Non-Episodic: Subsequence of percepts and actions makes one episode. In an episodic environment the action of agent just depends on that episode making such environment easy to handle because agents need not think ahead. Where as in non-episodic environment episodes cannot be formed and it is tough for agent to make an action ;

- Discrete vs. Continuous: When ever percepts and action occur in a discrete manner then the environment is discrete and in a continuous environment the percepts and actions are continuous. Playing a poker will be discrete environment and vehicle driving will be continuous environment;

- Static vs. Dynamic: If agent actions are only the ones which are making the environment to change its state then the environment is static else it is dynamic. Most of the real world systems are dynamic.

Because of multi-agents performance in different environment, they are widely applied in various disciplines. They are widely applied in manufacturing control and operation, transshipping containers, eco-system resource management, physical robots, power systems and many more fields. For the agents to successfully perform in all their applications, communication is the most important aspect. All the agents in multi-agent system need to cooperate and negotiate to achieve common goal. For this to happen with no ambiguity the agent communication has standards called Foundation for Intelligent Physical Agents (FIPA). 


\subsubsection{Agent Communication and Coordination}

As discussed earlier, agents have the ability to perceive, reason and act. For this to happen we assume that the agents have the ability to communicate. To achieve the organizational objectives, agents need to communicate with users, with system resources and other agents. For that they rely on speech act theory (Searle, 1969) and communicate using special communicating language. Knowledge Query and Manipulation Language (KQML, Mayfield et al., 1996) is first agent communication language developed by US government's ARPA knowledge sharing effort [4]. It is actually the language and protocol used to exchange knowledge. By incorporating KQML aspects more advanced agent communicating language is developed called FIPA ACL which has the possibility of using different content languages and conversation management.

Coordination is a very important aspect in multi-agent communication because of various reasons like (1) conflicts caused within agents because of their goals, (2) independent agent goals, (3) different agent capabilities and knowledge, and (4) rapidly achieved goals if others work on them [4]. Reducing resource contention, avoiding livelock, deadlock and maintaining applicable safety conditions can be achieved by agent coordination [6]. Extent of coherence of the system depends on how well the system can coordinate their behaviors and activities. Figure 1-1 shows how agents coordinate their behavior and activities to achieve coherence. 


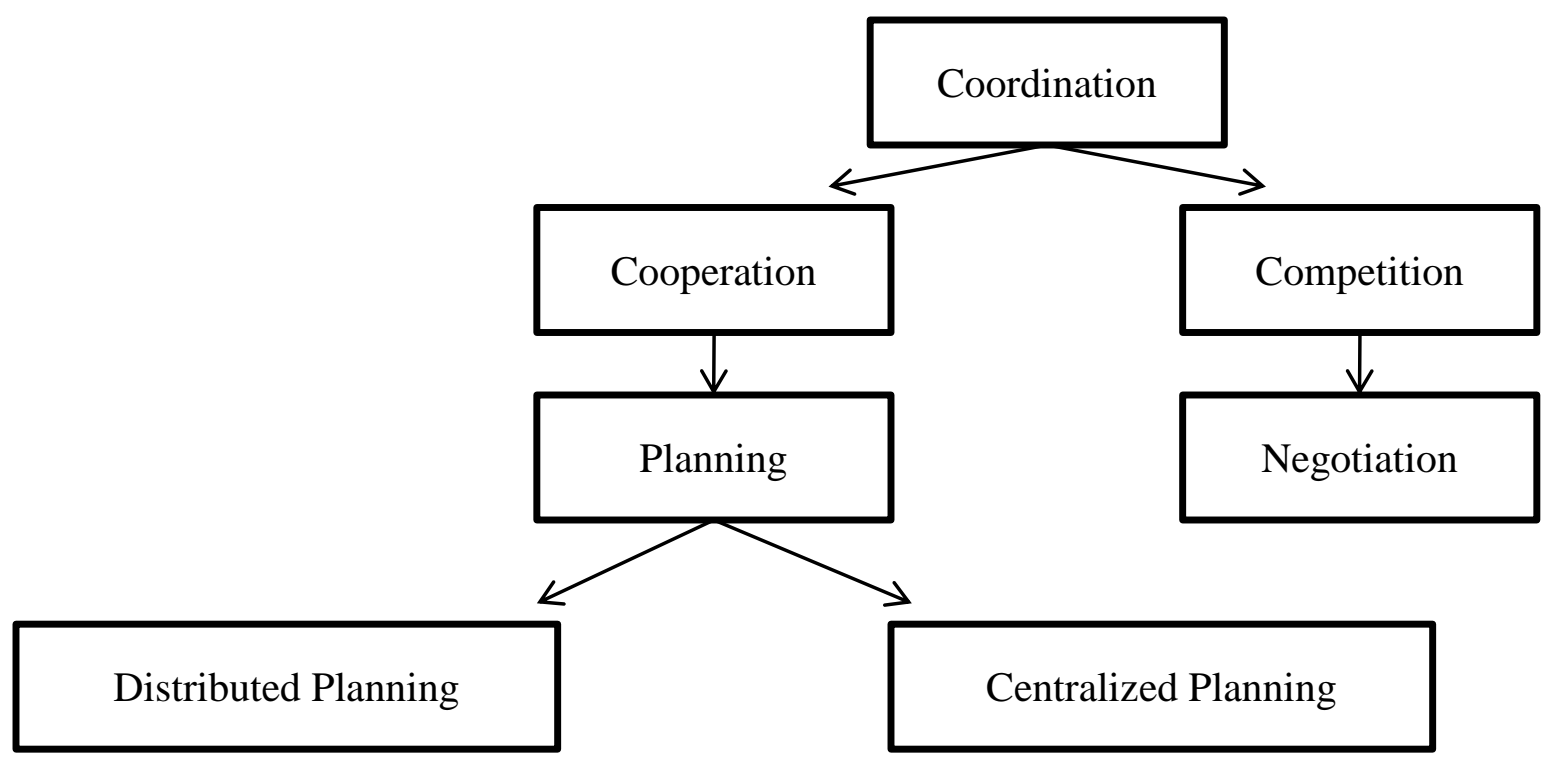

Figure 1-1: A taxonomy of some of the different ways in which agents can coordinate their behavior and activities [6]

Different dimensions of the meaning like syntax, semantics and pragmatic associated with communication need to be considered for the message to understand and to be understood [6]. There is also a need for communication to be defined at various levels so that the agents at different level can communicate. This brings in the need for different message types like assertions and queries. Agents have different abilities to be considered as active or passive agents. Irrespective of whether an agent is active or passive it must be able to accept information. For an agent to be considered as passive it should be able to receive the query and send a reply. For it to be considered as an active agent, it should be able to issue query and make assertions [6].

Communication protocols are specified at different level with lowest level being the interconnection method, middle level specifying the syntax of the information and top level mentioning the meaning of the message. Depending on the communication protocol there can 
be a single sender and single/multiple receiver(s). The protocol specified by the data structure will have fields such as (a) sender, (b) receiver(s), (c) language in the protocol, (d) encoding and decoding functions, and (e) action to be taken by the receiver [6]. As mentioned before KQML and FIPA ACL are the two agent communication languages, the latter being the advanced and recently developed version compared to the former.

\subsubsection{KQML}

KQML is basically both message format and message handling protocol to support runtime knowledge sharing by agents in multi-agent systems [10] as shown in Figure 1-2. It is developed by ARPA supported by knowledge sharing effort intending to support the interaction between the intelligent agents [11]. By using common syntax, semantics and pragmatics for the communication between agents most of the difficulties can be solved. Several basic communication protocols can be supported by KQML [11]. In KQML all information for understanding the message content is included in the message pattern itself [6].

KQML has LISP-style syntax and has some basis in speech act theory [9]. The general format of KQML language is:

(KQML performative Name

$$
\begin{aligned}
& \text { : sender S } \\
& \text { : receiver R } \\
& \text { : content C } \\
& \text { : language } \mathrm{L} \\
& \text { : ontology O }
\end{aligned}
$$




$$
\begin{aligned}
& \text { : reply-with } \mathrm{X} \\
& \text { : in-reply-to Y) }
\end{aligned}
$$

The above message says that the message is sent to $\mathrm{R}$ from $\mathrm{S}$ in response to the previous message identified by $\mathrm{Y}$ and any message in response to this message should include in-reply-to $\mathrm{X}$. The content $\mathrm{C}$ uses the language $\mathrm{L}$ and whose terms are taken from the ontology $\mathrm{O}[9]$.

Recently FIPA ACL is more employed for agent communication than the KQML in most of the agent based platforms.

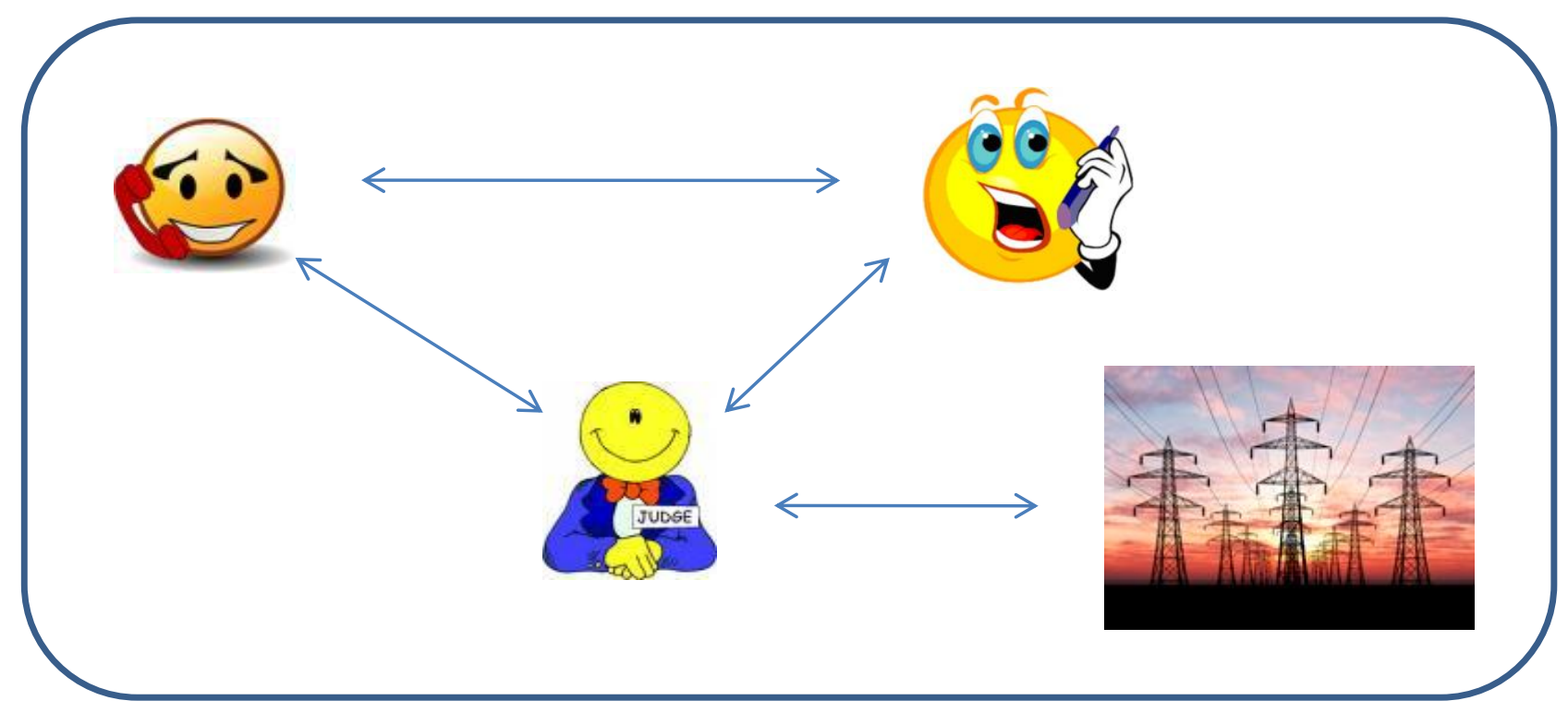

Figure 1- 2: KQML communication protocol

\subsubsection{FIPA - ACL}

FIPA was formed in 1996 for developing standards for heterogeneous and intelligent agents and agent based systems [12]. Later in 2005, IEEE accepted FIPA as its eleventh standard committee and FIPA is now an IEEE Computer Society Standards Organization promoting agent technology [12]. Lately a lot of FIPA's work is aiming towards integration of agent based 
systems with non-agent systems, taking agent technology to the next level. FIPA specification covers various agent technology aspects namely Agent Management, Agent Communication and Agent Message Transport.

FIPA's Agent Communication specification includes message exchange interaction protocols, speech act theory based communicative acts and content languages. An agent communication language (ACL) message specification describes a set of message types and their pragmatics. ACL message can typically contain any number of parameters but the compulsory parameter in all the messages is the Performative parameter. However, most of the messages are expected to have sender, receiver and content parameters depending on the situation. Other than FIPA ACL message parameters, few specific implementations can freely include user-defined message parameters [13]. Various FIPA standard message parameters are shown in the table below:

Table 1- 1: FIPA ACL language parameters and their interpretation

\begin{tabular}{|c|l|}
\hline Type of Message Parameter & \multicolumn{1}{|c|}{ Meaning of Message Parameter } \\
\hline Performative & It denotes the type of communicative act \\
\hline Sender & It denotes the participant of the communication, in specific \\
\hline Receiver & It denotes the receiver of the message \\
\hline reply-to & It denotes the identity of the agent to which the reply to the \\
\hline Content & It denotes and includes the content of the message \\
\hline Language & It denotes the encoding scheme of the content of the \\
\hline & message \\
\hline
\end{tabular}




\begin{tabular}{|c|l|}
\hline Encoding & $\begin{array}{l}\text { It denotes the specific encoding of the message content } \\
\text { language expression }\end{array}$ \\
\hline Ontology & $\begin{array}{l}\text { Its denotes the ontology used to give the meaning for the } \\
\text { expression used in the content expressions }\end{array}$ \\
\hline Protocol & $\begin{array}{l}\text { It is an optional one denoting the interaction protocol used } \\
\text { by agent to communicate in ACL language }\end{array}$ \\
\hline conversation-id & $\begin{array}{l}\text { It denotes an expression which in specific identifies the } \\
\text { sequence of ongoing conversation }\end{array}$ \\
\hline reply-with & $\begin{array}{l}\text { It denotes the expression used by an agent responding to } \\
\text { the message to identify the message }\end{array}$ \\
\hline in-reply-to & $\begin{array}{l}\text { It denotes the reference to the previous message for which } \\
\text { this message has been sent as a reply }\end{array}$ \\
\hline reply-by & $\begin{array}{l}\text { It denotes the preference of the sending agent in terms of } \\
\text { date and/or time by which the reply is expected }\end{array}$ \\
\hline
\end{tabular}

In a typical ACL message the parameters can occur in any order. As mentioned earlier every ACL message need not have all the parameters and can also include user defined parameters. A sample ACL message is shown below: 


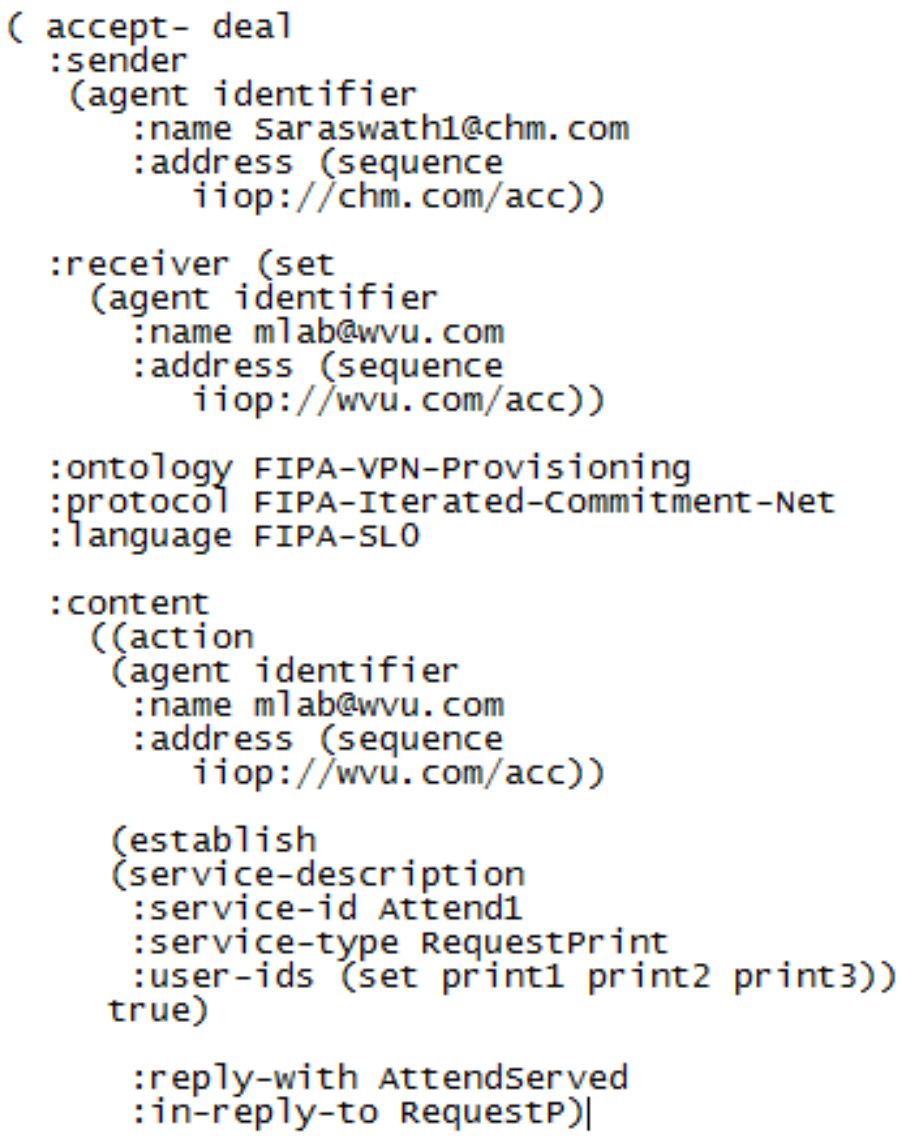

Figure 1- 3: A sample ACL message

The ACL message example in the Figure 1-3 contains few of the ACL message parameters. It is an 'accept-proposal' performative and also explicitly specifies the sender and receiver of the message. ACL message parameters such as ontology, protocol, language, content, reply-with and in-reply-to parameters are also being employed.

In this thesis multi-agent system management is provided by FIPA agent communication via ACL language. Java Agent Development Framework (JADE) is a Java based agent platform which abides to FIPA standards for agent communication. JADE is recently employed in most of the multi-agent systems development, so as in this thesis. 


\subsection{Smart-Grids}

In general, Smart Grid is the term used to refer to an entire power system i.e. generation, transmission and distribution networks, which has few special attributes making power system more reliable, efficient and productive. This basically involves embedding advanced technology for measurement, controlling and monitoring into the traditional grid and enabling highly developed communication technologies. Energy Independence and Security Act (EISA) of 2007 came up with federal policy under title XIII which calls for smartgrid development and deployment [15]. As a result of which the Department of Energy (DOE) established various standards for Smart Grid implementation. The central idea of this smartgrid initiative is to support the modernization of United States's electricity transmission and distribution system to maintain a reliable and protected electricity infrastructure that can meet upcoming demand growth [15]. The Smart Grid is expected to have certain key features as follows: [15] [16] [17]

- Dynamic participation on demand side

- House all generation and storage options

- Enable new products, services and markets

- Provide power quality for latest generation

- Asset utilization

- Self healing capability - Operate resiliently against attack and natural disasters Let us now emphasize briefly on the above key features of the Smart Grids:

A. Dynamic participation on demand side: This character is the concept of "real time pricing" or "Demand Response" in which the consumer at home/industry/commercial complexes is given a choice of reducing their loads or shifting their usage to non-peak 
demand time where the price of energy is low. This involves implementation of advance metering infrastructure which is capable of bi-directional communication. Multi-agent technology can also be implemented at various levels to embed knowledge into the grid and implement dynamic participation by customers.

B. House all storage and generation units: This feature reflects the encouragement of distributed energy resource accommodation as much as possible without disturbing the integrity of the grid in the smartgrid initiative. Employing distributed generation units makes system more complex and because of multi-directional power flow, traditional protective schemes must be greatly modified to retain the stability and security of the power system. The motto behind this to make the generation decentralized so that the end customer will be unaffected with the failures in the main generation units. The local generation units also help the local loads to tailor power directly from them reducing the congestion on the power lines. As mentioned earlier, decentralization of power generation will raise a lot of security and protection concerns in the power grid which needs to be addressed.

C. Enable New Services, Products and Markets: Since the Smart Grid is going to have many features which are not present or merely present in today's power system, there comes the necessity for the manufacturing of novel products to support the Smart Grid. This is going to influence the productivity, marketing and hence the economy of the manufactures and service providers [16].

D. Provide power quality for latest generation: With increase in the use of energy efficient devices like fluorescent lights, smart appliances etc., which are considered to be nonlinear by nature, power quality will be a vital preference. This non-linear loads inputs 
harmonics in the system and results in poor power factor. The usage is expected to be increased and hence the harmonic components of voltage and current resulting in ramping of transformer overload. All these can be avoided with high power quality which can be achieved via Smart Grid technology.

E. Asset utilization: This stands as an overriding reason for many utilities for developing Smart Grids. Maintaining power assets like main generating units, backup generating units, main and backup substations etc., will be a major factor which affects the power cost for the consumers and over all carbon foot-print print for the utilities. Considering the optimization of assets utilization and operating the power stations efficiently is hence considered as an important feature of Smart Grid.

F. Self-Healing Capability and Security: This character directly addresses the attribute of the Smart Grid to be reliable and resilient. Enabling peer-peer communication in the system helps to separate faulty part from the healthy parts of the network and enabling the healthy parts to operate independently forming islands. Anticipation of the faults will also be a part of it which needs vigorous innovative technology which predicts the fault time and location. Automation of any process demands high security from cyber attacks. Though it is tough to avoid natural disasters it is really important that the damage should be minimized and reduce the discontinuity of the power service.

Apart from trying to implement all the above mentioned features to achieve smart grid, there exists a real need for an extensive socio-economic analysis to study the current grid status and smart grid cost and benefits [44]. A study has been made me look into the gap between the present grid and expected smart grid. Table 1-2 presents the gap and proposed smart grid 
solution.

Table 1- 2: Gap and proposed smart grid solution [44]

\begin{tabular}{|c|c|}
\hline Gap & Solutions \\
\hline Technology & $\begin{array}{l}\text { - Advanced Meter Infrastructure } \\
\text { - Information Technology Integration } \\
\text {-Demand Response } \\
\text { - Distribution Management Systems } \\
\text { - Distributed Energy Resource } \\
\text { - Transmission Management Systems }\end{array}$ \\
\hline $\begin{array}{l}\text { Regulatory/ } \\
\text { Governmental }\end{array}$ & $\begin{array}{l}\text { - Inclusion of Smart Grid investments in rate base } \\
\text { - Rate structures that encourage DER, DR and } \\
\text { differentiated PQ } \\
\text { - Federal and state funding of related R\&D } \\
\text { - Change in regulatory policy to create incentives } \\
\text { or remove dis-incentives for investment Deci- } \\
\text { sions based on socioeconomic analysis } \\
\text { - Recovery of Smart Grid-driven stranded capital }\end{array}$ \\
\hline Consumer & $\begin{array}{l}\text { - Consumer Education } \\
\text { - Access by all consumers to the electrical market } \\
\text {-DER interconnect standards } \\
\text { - HANs and consumer agents } \\
\text { - Standardized transfer of market information }\end{array}$ \\
\hline
\end{tabular}

This thesis work mainly focuses on the demand response or dynamic demand participation attempting to reduce the peak load and thereby reducing the congestion on power lines. Due to increase in power usage there is a need for the expansion of power units to serve the load which involves investment of a lot of capital. Adopting distributed resources and dispatching the load considering peak hours and non-peak hours may solve the problem or delay the urgency of new installations. Multi-Agent technology is adopted to embed knowledge into the grid.

Because of its importance and evolutionary changes that brings into the power systems, there is an extensive research federally supported on Smart Grids. National Rural Electric Cooperative has summarized industry research efforts in its report which presents the glimpses of Smart Grid 
related research work [17]. In 2001 Electric Power Research Institute (EPRI) initiated IntelliGrid a Smart Grid initiative project focusing on the development of smart power system which is integrated with advance communication, computing and electronic devices[18]. The IntelliGrid provides utilities with the methodology, tools and recommendations for standards and technologies when implementing systems such as advanced metering, distribution automation, demand response, and wide-area measurement [18]. Presently EPRI is implementing five different projects namely IntelliGrid architecture; Fast simulation and Modeling; communication for DERs; consumer portal; and advance monitoring systems.

EPRI advance distribution automation (ADA) project envisions future automated distributed system with Intelligent Electronic Devices (IEDs) which makes the distribution system more improvised with the capability of reliability, capacity utilization and better customer service [19]. Modern Grid Strategy previously named as Modern Grid Initiative was developed in 2005 by U.S. DOE, Office of Electricity Delivery and Reliability (OE) and National Energy Technology Laboratory (NETL) has the major goal to accelerate the electric grid modernization. The team renamed itself as Smart Grid Implementation Strategy (SGIS) attempting to evaluate and implement smart grid planning; smart grid business case development at various levels; engineering analysis of smart grid deployment; evaluation and communication of smart grid work with stakeholders; and integration of SGIS work with other organizations [20].

GridWise Alliance is developed by DOE in 2003 envisioning the future electric delivery [21]. Its policy is to increase national vision about smart grids through advocacy of well reasoned smart grid policies at the federal, state and regional levels [22]. GridWise Architecture Council is a 
major working part of GridWise project which includes a team of industry leaders which shape the architecture of highly interactive and intelligent electric systems [21]. Advance Grid Application Consortium (Grid Apps), GridWorks, Distribution Vision 2010 and California Energy Commission initiated Public Interest Energy Research (PIER) Program few other smart grid initiative projects which are aimed to expedite the transformation of present electric system to highly advanced and powerful grid know as Smart Grid.

\subsection{Problem Statement}

In this thesis a multi-agent system based (Demand Dispatch?) peak demand reduction algorithm is developed as a part of smart grid initiative research work. Reduction of peak demand is achieved using controllable load dispatch and distributed energy resources (DER). Agents at various levels like agent at load serving entity, DER agents, load agents, etc. heuristically attempt to achieve the peak demand reduction.

In most of the utilities' service area demand response or real time pricing is employed to encourage electricity usage during non-peak hours so that the peak demand will be reduced and hence the overall price of the electricity. Peak shedding is other major strategy to achieve peak demand reduction. In this thesis neither the real time pricing nor prioritizing the load for shedding is considered hence peak demand reduction is a critical problem. Reduction of the peak demand at least by $15 \%$ is the target 
The assumptions made in this thesis to accomplish peak demand reduction using controllable loads are:

- Controllable loads respond quickly to the signals sent by the load aggregator

- Transients caused by the Distributed Energy Resources are taken care of

- There exists at least certain percentage of loads in the considered service area which are controllable with no notice of service interruption or minimal interruption

\subsection{Outline}

This section outlines the remaining chapters of this thesis

Chapter 2 describes the glimpses of research in the field of multi-agent systems applied in various engineering fields and specific power system issues. It also focuses on research related to the smart grids and demand management.

Chapter 3 elucidates the simulation software packages employed, their advantages and

limitations. It also shows the simulation setup and case studies employed for peak demand reduction.

Simulation and results of the approach are presented in Chapter 4. Conclusions and scope for the future work are discussed in Chapter 5. 


\section{Chapter 2}

\section{LITERATURE REVIEW}

This chapter presents the overview of the current and previous research work and implementation related to this thesis area. This chapter will focus on work related to multi-agent systems applied in various disciplines and specifically in power systems for demand response, distributed generation and load management. We are also going to emphasize on work related to load management techniques, distributed generation employed for peak demand reduction.

\subsection{Multi-Agent Systems}

As discussed in earlier chapter a Multi-Agent System (MAS) forms a network of distributed intelligence embedded into any application to solve the problem efficiently. Though single agents do possess intelligence, due to the inheriting distributed nature of most of the real time systems, it becomes almost impossible for one agent to control and monitor the system. Automation of all real time systems is becoming a priority and hence advanced technologies like MAS technology is becoming a part of various applications.

\subsubsection{Multi-Agent Systems Applications Overview}

Manufacturing industry faces a lot of competition in terms of reducing the development time of the new products and in addition diversity of consumer's needs makes it very difficult to 
assemble and optimize the process plans for the planners [23]. A MAS technology based Computer Aided Process Planning (CAPP) distributed environments were effectively developed in [23]. The whole planning problem is split into simple modules of problems. The systems consists of nine categories of agents each have their own knowledge and reasoning. These agents collaboratively solve their sub-problems with their knowledge, achieving the process planning efficiently. Figure 2-1, describes the communication and collaboration of agent to achieve task:

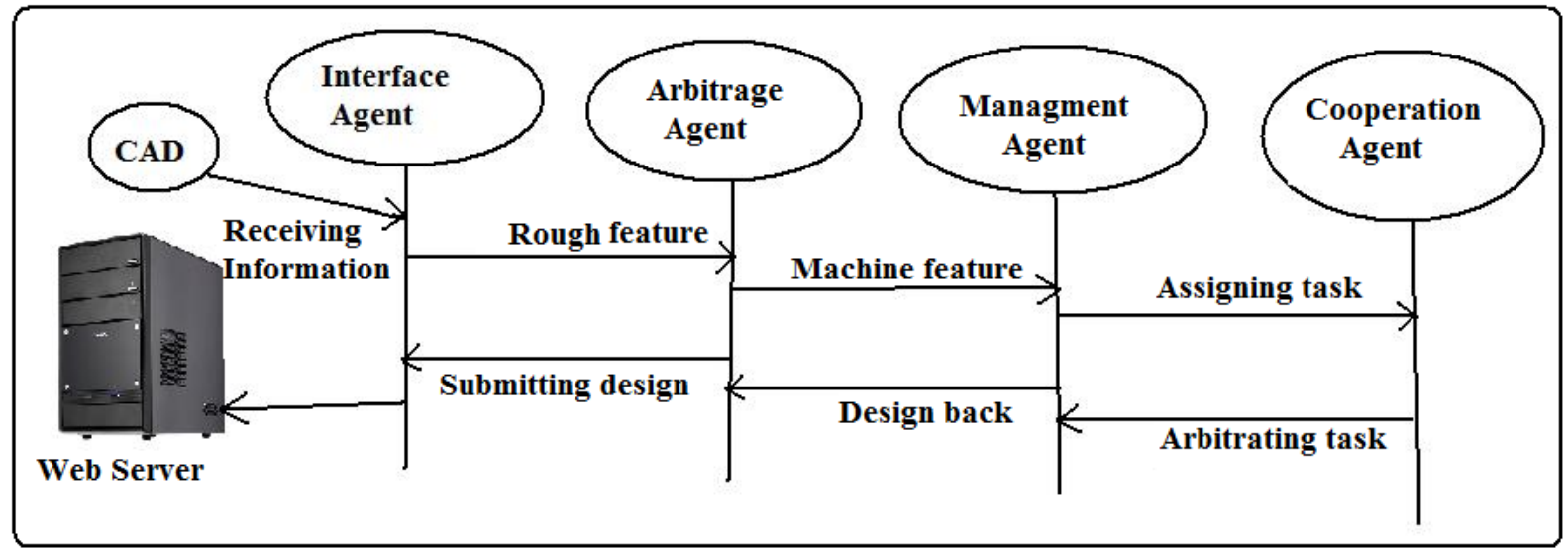

Figure 2- 1: System flow chart for MAS based CAPP for manufacturing [23]

The advancement in vehicular technologies brings in the transport system applications development in terms of providing information for safety and convenience. MAS can be affectively applied to transport system which requires most relevant, interactive and instantaneous information during their travel [24]. Unlike the traditional client-server (CS) communication used to obtain related information, which is prone to high traffic issues and network contingency issues, MAS technology can efficiently overcome all these concerns [24]. In [24], authors adopt MAS, using mobile-agent paradigm, in order to optimize the services research and composition in transport business. System consisting of five kinds of software agents is deployed and they are coordinated to solve the problem. The results have proved that 
the MAS based model have fast response and has optimized benefits over traditional CS model.

The development of urban traffic demands for high performance and complex function of traffic signal control which is expensive because of the concerns associated with operation, maintenance, upgrading and expansion [25]. The paper [25] presents MAS based model for traffic signal control. A new signal control model is developed which consist of various modules like main control module, lamp control module, input module, voltage/current monitor module and failure detecting traffic control systems module. For efficient and reliable traffic control operation, traffic control-application specific operating systems runs on the main module [25]. The traffic controller becomes an agent host where different agents reside at different times in response to different traffic conditions.

Due to the advantages of MAS and their effectiveness in solving various issues, they are widely applied in Electric Power Systems (EPS) to impart intelligence in the system. There exists an extensive research work in application of MAS to various fields of power systems like fault reconfiguration and restoration, protection coordination, electric power markets, distributed generation integration, and demand response. The further part of this chapter briefly emphasizes on few general application of MAS in EPS and in-detail focus on research related to this thesis' work.

Reliability of the EPS stands a major priority, and to achieve that, effective fault location and restoration techniques need to be employed. Authors in [26] have developed MAS based fault detection, diagnostics and prognostics for war ship. The necessity of intelligent software agents 
and their performance over traditional diagnostics schemes employed in war ship were elucidated. The proposed agent architecture has two types of agents named diagnosis agents and prognosis agents. The diagnosis agents helps to monitor the system and the prognosis agents predicts the failures and can hence help to avoid system faults. A test case on permanent magnet synchronous motor (PMSM) is considered and particle swarm optimization technique is employed to demonstrate fault diagnostics and detection.

The operation of electricity market is a vital part and automation of which requires meticulous attention. MAS seize the attention of system operators for the automation and efficient power market transactions. In [27] authors demonstrate the implementation of MAS in power markets and compared pay-as-bid and uniform bidding strategies using MAS. Using multi-agent system they have proved that in the pay-as-bid auction agents bid close to the market price and reduces market price and price volatility significantly compared to uniform bid strategy. Q-learning strategy is employed to teach auction behavior to the agents, the strategy helps agents to take a decision with incomplete information and with direct interaction with the environment. It is also been observed that the agents behave differently while employing different strategies, making them the right choice for the automation of energy markets.

\subsubsection{Multi-Agent Systems in Smart Grids}

The idea of the smart grid is to modernize the EPS so that it monitors, protects and automatically optimizes the operation of the elements interconnected to it [28]. As discussed earlier, the smart grid is expected to have major extraordinary characteristic like self healing, accommodation of DER, enabling new products and services, provide power quality, optimize assets, anticipate and 
defend system disturbances, and cyber security and resilience against attacks. Implementation of MAS technology can implicitly help to achieve most of the above mentioned functionalities. In [28] the author elucidates how advance computer aided technologies like MAS can help to achieve smart grid goals. The proposed methodology for market interoperability of smart grid helps to achieve the global optimization of energy consumption hence can significantly reduce the peak demand, thereby avoiding the transmission expansion costs [28]. It predicts how MAS can be employed in power markets for automatic negotiation and coordination, which can reduce the market period enabling to handle DER easily.

The attempt to make the power system a self-healing one is to make it resilient to power flow unbalance, power outages, and voltage instability and security failures [29]. Authors in [29] have presented design issues that are appropriate in developing MAS for grid to make it vulnerable and self-healable. The design considers various agents at different level coordinating as shown in the below figure to achieve tasks:

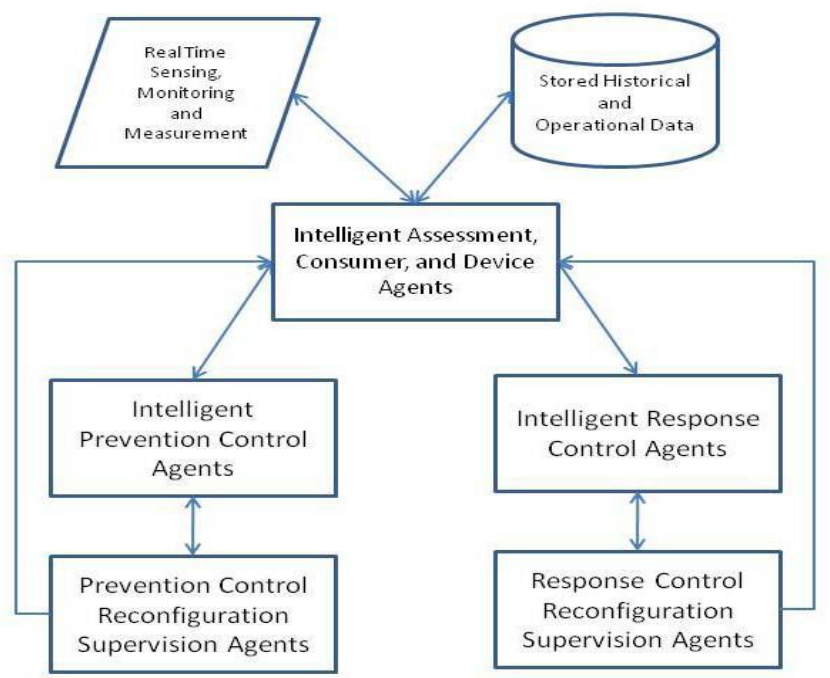

Figure 2- 2: Communication flow in MAS for smart grid simulation [29] 
In [30] authors propose a fault location algorithm for a distribution system in a smart grid. The algorithm uses MAS, where agents are located at various node locations. The agents have the local feeder information and when fault occur the zero transient reactive power is calculated using zero transient voltage and current. The agents communicate the information and depending upon the direction of the reactive power flow the fault location is identified [30]. The system results are claimed not only to be accurate but also assures the robustness of the system.

Accommodating as many DERs as possible into the grid helps in microgrid management, intelligent load control, and smart charging applications. Author David A. Cohen in paper [31] explains how DERs integrated simulation software; GridAgents ${ }^{\mathrm{TM}}$ makes use of multi-agent system to enable the deployment of communities and eco-systems of smart grid. GridAgents ${ }^{\mathrm{TM}}$ is a novel, advanced software foundation desired to integrate with SCADA and internet based applications for developing optimized DER control in varying operational configurations such as microgrids, demand response automation, Distribution Network Control, and intelligent monitoring applications [31]. Agents at different levels as shown in the figure below coordinate and help to achieve the above mentioned goals. 


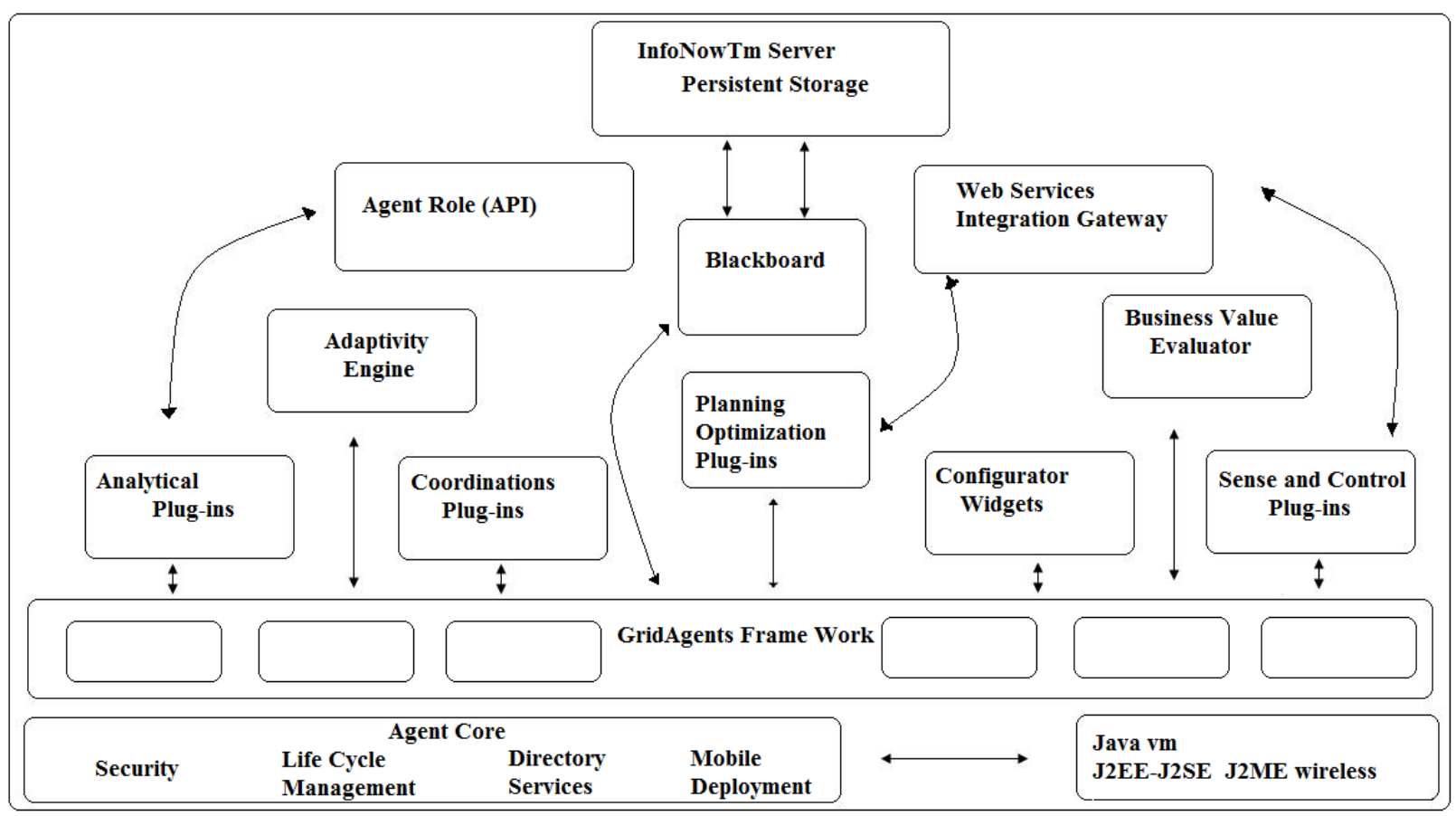

Figure 2- 3: DER integration plan for advanced distribution operation

Dispatch of customer load controllers for compact network load alleviation is demonstrated in the paper as an example. Various agents at load level and transformer level are managed by management agent to accomplish the alleviation.

\subsubsection{Multi-Agent Systems in Demand Management}

Dynamic load management of the power grid is essential to make better and more cost-effective use of electricity production capabilities and to increase customer satisfaction [32]. Demand management plays a critical role not only in the reduction of electricity usage price for the consumer but also to reduce the congestion on the power lines, peak demand, power system expansion expenses and much more benefits contributing to the smart grids. Work related to application of MAS in demand management is discussed in this section. 
In [32] authors Frances Brazier et al. proposed a load balancing technique based on multi-agent system negotiating scheme. The idea is to establish a flexible negotiating scheme for individual costumers, utilities and power generating units to optimize the overall electricity cost. The paper proposes different protocols for negotiation and a compositional development system for MAS in DEsign and Specification of Interacting Reasoning (DESIRE) software. The intention of load management is to smoothen the total peak load by managing a more appropriate distribution of the electricity usage among consumers. Agents at utility level and consumer level are deployed in this paper which negotiates prices as a part of peak demand reduction. Monotonic concession protocol is applied for the load management problem and assumption made is consumer behavior can be influenced by financial gain [32]. Three methods of negotiation namely offer, request for bids, and announce reward tables methods in the load management domain are proposed.

Demand response must be adopted in load management to make the future power system to adequately use all the available resources in order to satisfactorily provide quality of service to the customers [33]. In [33] authors presented an approach to deal with all issues associated with integration of available resources in load management. A multi-agent system which is capable of modeling demand side players and their strategic behavior is demonstrated [33]. The case study of a utility company is shown where reduction of load curtailment is obtained because of previously established load flexibility contracts with their customers. MASCEM; a MAS based electricity market (EM) simulator, has been employed. Demand side players which have active role in EM in two different ways; one in which they participate in energy transactions and the other in which they participate in demand response programs, are deployed in MASCEM. A case study was made on 2040 future electric network as shown in the figure below: 


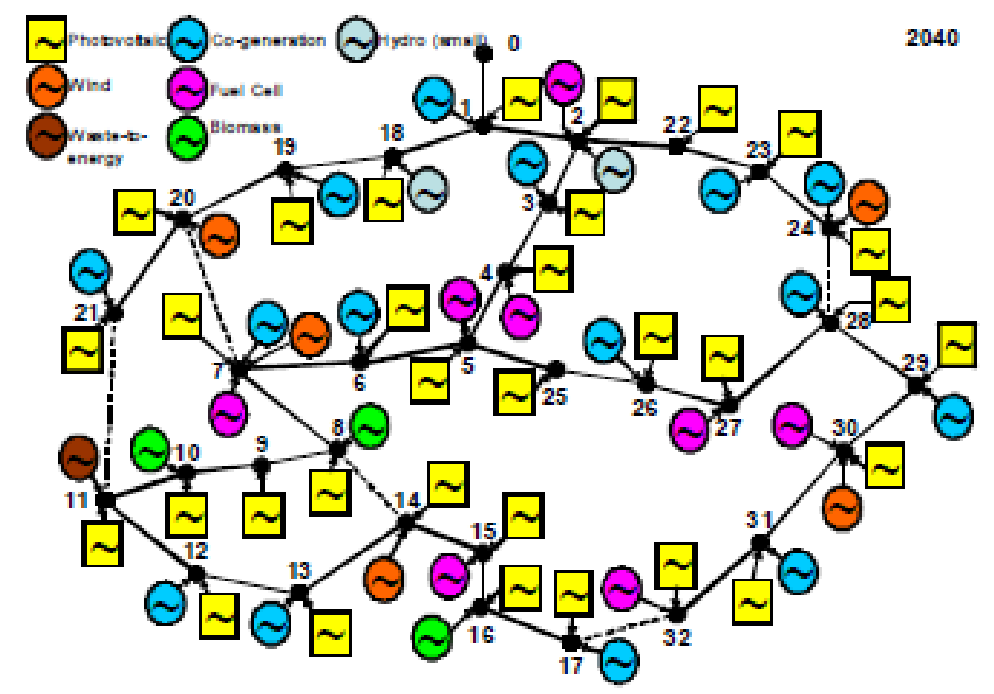

Figure 2- 4: Future anticipated electric network 2040 [33]

Short circuit in the line $0-1$ has been simulated and the network is operated for 3 hours to study the demand response behavior. Distribution companies utilize DERs to supply the load and limit the load curtailments [33]. The results in the paper conclude that the usage of flexible contracts in demand response considerably decreases momentary value of lost loads.

In paper [34] authors propose PowerMatcher; a multi-agent based control concept and software package, concept which they claim to be very successful for virtual power plant control leading to peak load reduction up to 30-50 \% without any intrusion on comfort for customers. They basically attempt to coordinate demand and supply in electric network with a high share of distribution generation. A virtual power plant (VPP) with ten micro-combined heat and power ( $\mu$-CHP) units are used in field test to study the performance for peak load reduction [34]. 


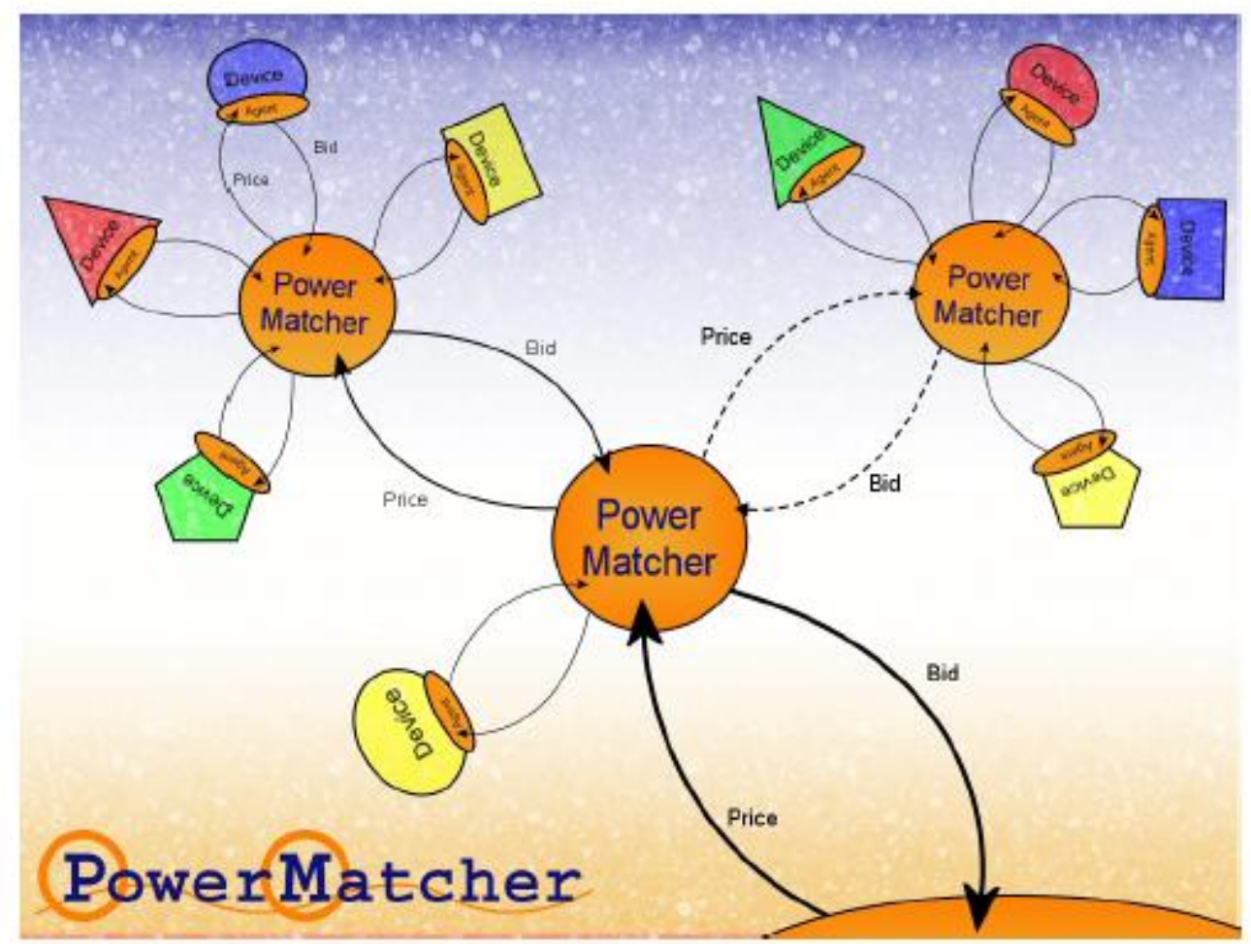

Figure 2- 5: PowerMatcher hierarchy based architecture [34]

The MAS in PowerMatcher adopts unified microeconomics and control theory, in which each device is represented by control agent which tries to operate the process associated with device in an economically optimal way [34]. The electricity consumed or produced by the device is bought, respectively sold, by the device agent on an electronic exchange market in which PowerMatcher as depicted in Figure 2-5 coordinate demand and supply to achieve global and local optimization. In the field test VPP nodes are installed in each $\mu$-CHP unit. The cluster of the units communicates among local infrastructure (thermostat, e-meter, $\mu$-CHP) and with the server agent (PowerMatcher) to reduce peak demand [34].

Introducing the price elasticity of demand has been a traditional approach to reduce congestion chargers and also to mitigate the market power of generation and transmission companies [35]. 
In the paper [35], authors use a multi-agent based Electricity Market Complex Adaptive System (EMCAS) to explore the impact of consumer price elasticity of demand. The percent change in load in response to percentage change in price is termed as price elasticity of demand. Research has proved that the residential customers barely respond to the increase in electricity price and large consumers are relatively price sensitive [35]. In EMCAS, the customers submit day-head demand to the load aggregators who, in turn, submit day-ahead hourly demand bid to ISO and GenCos submit their day-ahead hourly offer to ISO. ISO does the optimal power flow and determines locational marginal pricing (LMP). The consumer/load aggregator can submit elastic or in-elastic demand bid [35].

In this paper case study has been presented considering four scenarios namely, base case, GenCo strategies without demand-side bidding, demand-side bidding without GenCo strategies, and demand-side bidding along with GenCo strategies. 11-node system is considered for the case study and demands were aggregated at 5 different nodes. The price elasticity impact depends on the price level at which consumers' exhibit price sensitivity; consumers could gain by a reduction in energy prices and also reduce the congestion charges. Some of the customers may face a higher cost due to their location in the network [35].

\subsection{Demand Management}

Extensive research has been performed in the field of demand management for various purposes. This segment presents briefing about research related to peak demand reduction using various strategies. 
In [36] authors K. Morgan et al. describes winter peak demand reduction scheme's (WPDRS) structure and its results. This scheme was introduced by the Irish Transmission System Operator (ITSO) as a demand side management scheme to reduce the substantial increment of peak demand and was opened to independently supplied customers [36]. The participants do not react to intermittent or economic signals, but they strictly follow the load reduction or on-site generation every day during the peak demand time (5pm to $7 \mathrm{pm})$ according to the contract terms [36]. Load profiles are determined using meter data and load reductions for the WPDRS are designed relative to the obtained load profiles. For the customers to participate in the program 15 minute metering must to available at the customer location. To make the scheme successful high penalties are imposed for non-compliance [36]. The payment comprises of three components: the reliability payment, the reliability rebate, and the energy payment which are calculated based on power usage parameters peak period baseline and energy benchmark ratio [36].

The committed level of power usage during peak demand time is set using baseline which is $80^{\text {th }}$ percentile of MW usage during peak demand period of the previous year. The reliability payment is determined for each day by the difference between the committed level and the baseline, multiplied by $€ 320$ / MW. The reliability rebate is calculated by the maximum amount by which the committed level was violated during the peak period, multiplied by $€ 3200$ / MW. Consumers earn energy payments at a rate of $€ 50$ / MWh every half hour if their energy consumption is less than the energy benchmark divided by four. The total payment will be the sum of the reliability payment and the energy payment, less the reliability rebate. This payment is accumulated over the month and also the scheme makes sure that the payment will never be less than $€ 0$, i.e. the consumers will never increase their bill after enrolling in the scheme [36]. 
Various methods are adopted to see the success of the scheme and the Figure 2-6 shows the demand curve after and before adoption of WPDRS.

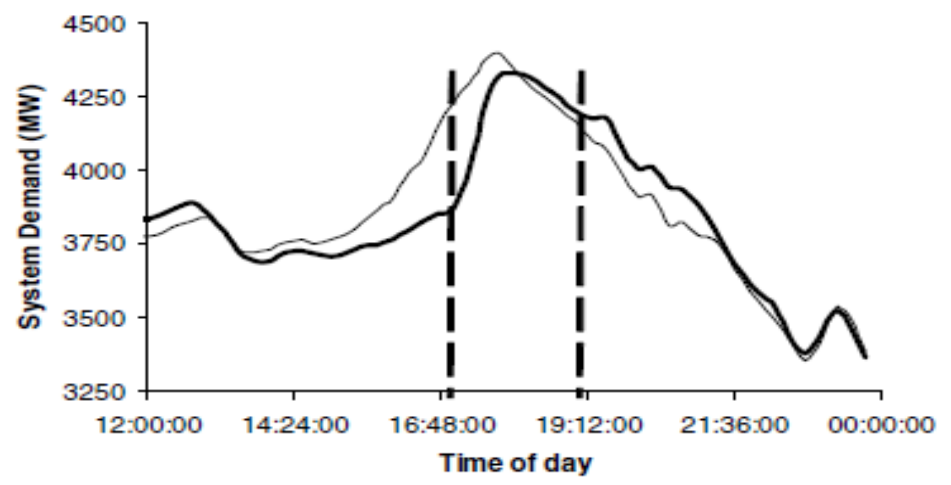

Figure 2- 6: Two load curves, showing the historical peak of $4400 \mathrm{MW}$ on $7^{\text {th }}$ January, 2003 (light curve, no WPDRS) and this winter's peak of $4329 \mathrm{MW}$, (heavy curve, with WPDRS) [36]

In [37] authors Richard E. Crane et al. presents how the use of separate storage devices for solar and off-peak electrical energy are desirable in reducing electricity demand peaks by storing offpeak power. Conventional way of operating solar cooling or heating system is that when the sun shines, the solar system satisfies the building instantaneous demand. Excess solar energy is put in storage. When the building demand exceeds the solar system's instantaneous capacity, stored energy is used to satisfy it. Auxiliary energy is used only when neither the collector nor the storage systems can provide sufficient energy to satisfy the building demand [37]. With the increase in the penetration of solar air conditioning customers, the above mentioned practice will contribute to the peak demand increment. Research shows that the significant cost increases will be incurred by the electric utilities due to the reduced load factors caused by this method of operating backup heating or cooling system on long run [37].

Authors in [37] describe different storage options, their behavior in different conditions and their contribution in reducing peak demand. Initially it has been concluded that introducing thermal 
storage of either an oversized or regular size is not an effective way of decreasing utilities peak load. Control methodology of different storage patterns along with solar air conditioning systems and their behavior which may considerably affect the peak demand are discussed. Storage device recharged during off-peak period to a level sufficient to satisfy demand during subsequent onpeak period based on expected temperatures and solar collection during that period is shown have smallest stand by losses and no-on peak demand [37]. However, the cost of the additional hardware is not proved to be weighed against the savings obtained through reduction of peak demand.

Authors John Byme et al. in [38] proposes dispatchable, peak shaving Photovoltaic (PV) arrays using storage units. With increase in investment on demand side management and their potential to avoid the expansion of power plants their rises the need to make PV arrays more efficient to serve the customers. In mid-Atlantic regions where demand peak occurs during 3:00 PM to 6:00 PM, the PV output can be increased by changing the orientation of wall angle of the arrays and dispatch ability can be integrated using direct load control devices [38]. Figure 2-7 explains the schematic of dispatchable PV arrays with storage units.

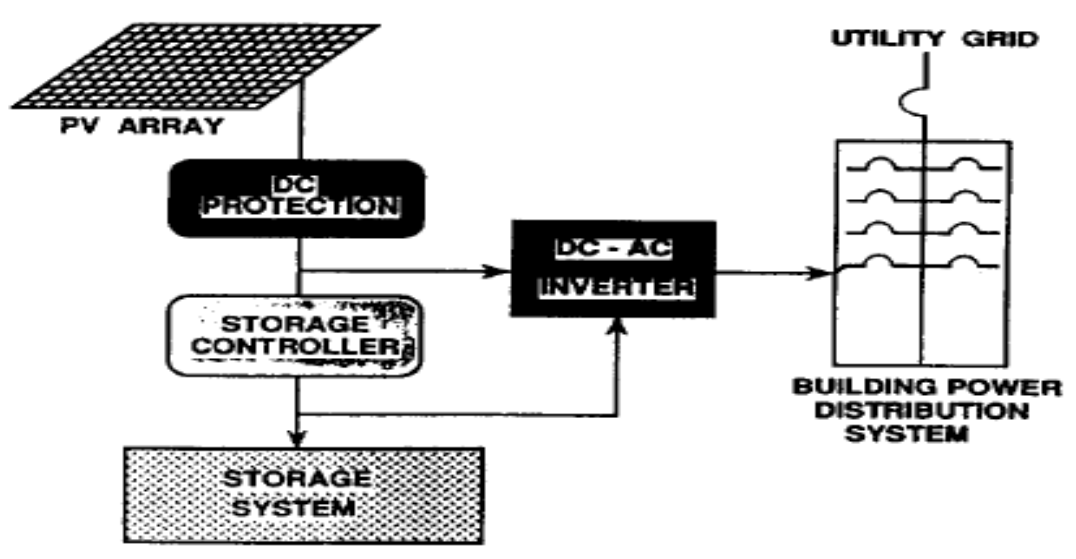

Figure 2- 7: Block diagram of dispatchable PV arrays using storage [38] 
The economic analysis made on $10 \mathrm{KW}$ PV units proved that there needs to be a partnership between customers and utilities to capture the full economic benefit of PV-DSM system [38]. The paper also discusses the advantages of PV units against battery-only dispatchable peak shaving units that there will be zero fuel cost for PV units, no energy purchase like battery units and higher economic value than the batteries [38]. Authors finally conclude that the proposed dispatchable PV peak shaving arrays are cost effective and efficient than supply side alternatives to control the peak demand.

In [39] authors A. J. Hoffman et al. introduces a novel technique for optimal adjustment of target peak level based on time series forecast of overall load of the complex and it is also shown how improper selection of target peak level can result in secondary peaks. Extensive research has proved that the commercial building electricity usage is the major contribution of energy usage and in which air-conditioning and lighting accounts for up to $80 \%$ of energy usage during peak demand time [39]. The algorithm proposed in the paper tries to optimize the peak demand of the building over a demand period of 15 minute, during a specific month.

The target peak demand is determined based on past four year's peak demand average and target saving factor. The 15 minutes demand period is divided into decision intervals of 30 seconds each. The control algorithm is executed by determining few parameters at the starting of every decision period and the parameters are [39]:

- Sheddability status of the equipment in the complex

- The current KVA, expected KVAh by end of the demand period, KVA that could be shed, KVAh added by turning on the equipment 
- Maximum time duration till which the equipment can be kept off

- KVAh during the demand period is determined based on KVA of individual equipment and base load. That will be subtracted from target peak demand to obtained KVAh need to shedded (KVAhreq)

- The equipment that has become unsheddable will be turned on

- If the above calculated KVAhreq is positive, the algorithm will find the equipment which can be shedded and will start shedding till the target is reached based on priority

- If KVAhreq is negative algorithm will select loads which can be turned on

- Should that target peak demand is achieved for the month the same target will be continued else it will adjusted upward

It has been shown how simplified equipment models can be used to provide satisfactory results for equipment behavior and also demonstrated how the prediction of total future demand can be used to sustain the target peak at an optimal level.

\subsection{Challenges for Peak Demand Reduction}

Lot of the research and implementation is focused on peak demand reduction using load shedding and demand response based on price signal. The problem with the prior approach is that in many utility service areas, according to the consumer service a commission, prioritizing loads/ customers is prohibited. Hence, the peak demand reduction using load shedding becomes no more a solution. 
The later approach of introducing price signal as a medium for peak demand reduction also becomes an inefficient option because of various reasons. First, there are still so many utility service areas where real time pricing or more tariff rates during the peak demand time does not exists, not at least to the residential customers. Secondly, the savings of the customers may be so less weighed to their commitment to the power usage reduction. Hence, a direct load control/dispatch method using an advanced technology like MAS can be adopted for peak soothing with minimal customer's effort and less inconvenience for the customers. 


\section{Chapter 3}

\section{SIMULATION SOFTWARE}

In this chapter we put some limelight on the software packages employed in this thesis work to design the multi-agent system platform and electric demand forecasting algorithm. Programming the multi-agent system greatly depends on the software package employed to do it. It is very important to pick the right platform to develop multi-agent system because, so designed multi-agent system is expected to interact with power system simulation entities which in most of the cases are developed using different software packages.

As a part of this research work various software packages used to simulate multi-agent system and power distribution system are scrutinized. For the requirement of this thesis work Java Agent DEvelopment framework (JADE) is utilized to develop multi-agent system and simulate their behavior. Various engineering toolboxes in MATLAB are employed to obtain the load forecasting model. It is very important to select appropriate software packages to successfully achieve interfacing between the MAS platform and PDS platform to study their interaction.

As mentioned earlier, two different layers exist while simulating power system embedded with intelligence. The figure below describes the architecture of simulation model 


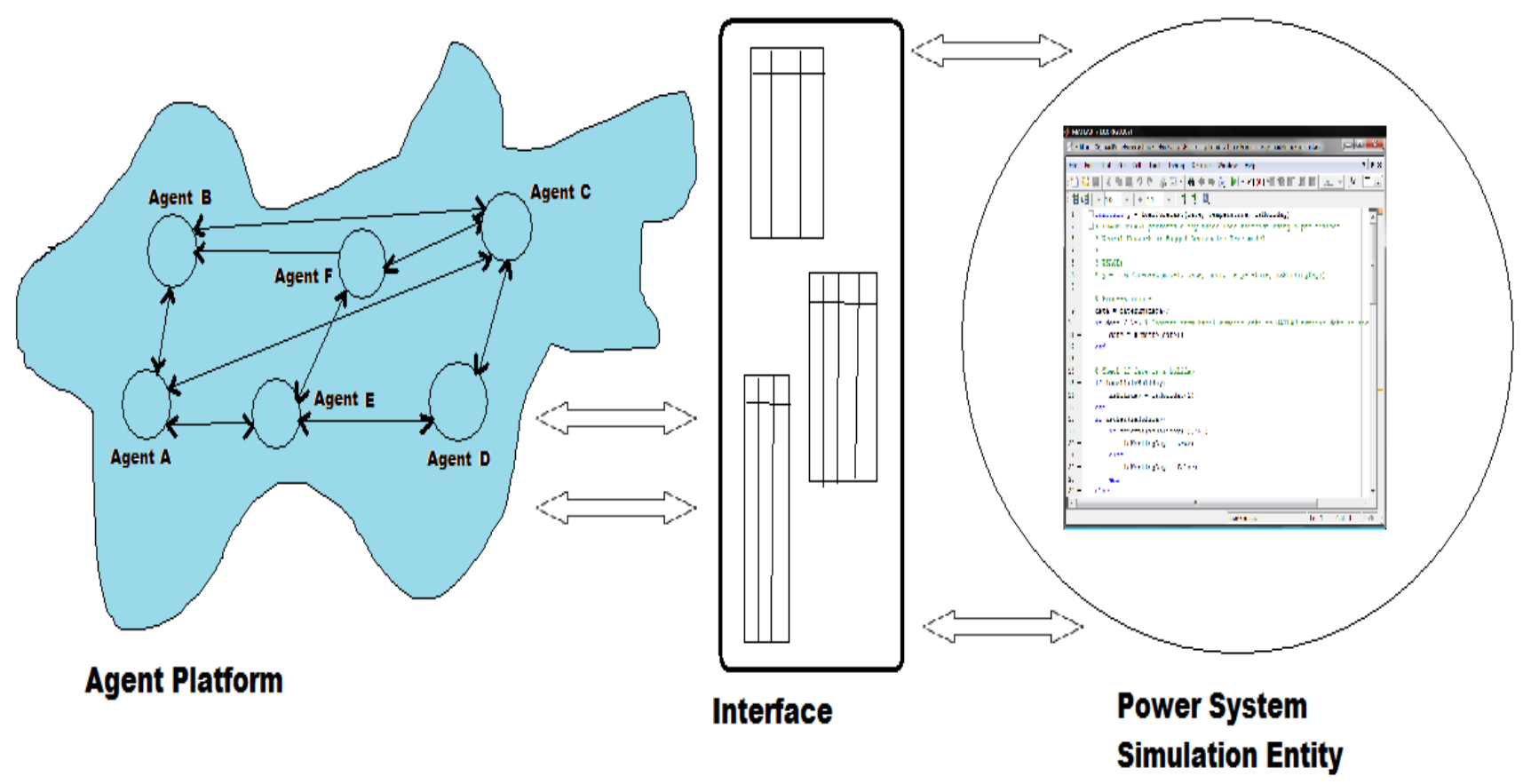

Figure 3- 1: Simulation Model Architecture

\subsection{MATLAB}

MATLAB developed by math works is high developed numerical computing environment. MATLAB (matrix laboratory) is developed in such a way that it is capable of solving computationally intensive tasks than with traditional programming languages $\mathrm{C}, \mathrm{C}++$, FORTRAN [41]. Apart from being a computing environment MATLAB is as a fourth generation programming language. It is used for matrix manipulations, plotting of function and data, implementation of algorithms, creation of graphical user interface, interfacing with the algorithms coded in different languages like $\mathrm{C}, \mathrm{C}++$, Java and FORTRAN.

MATLAB provides a range of computational toolboxes which can be used in engineering, science and economic analysis. MATLAB also offers a simulation package called Simulink 
which is meant for graphical multi-domain simulations and Model based design. Figure 3-2 depicts MATLAB GUI appearance.

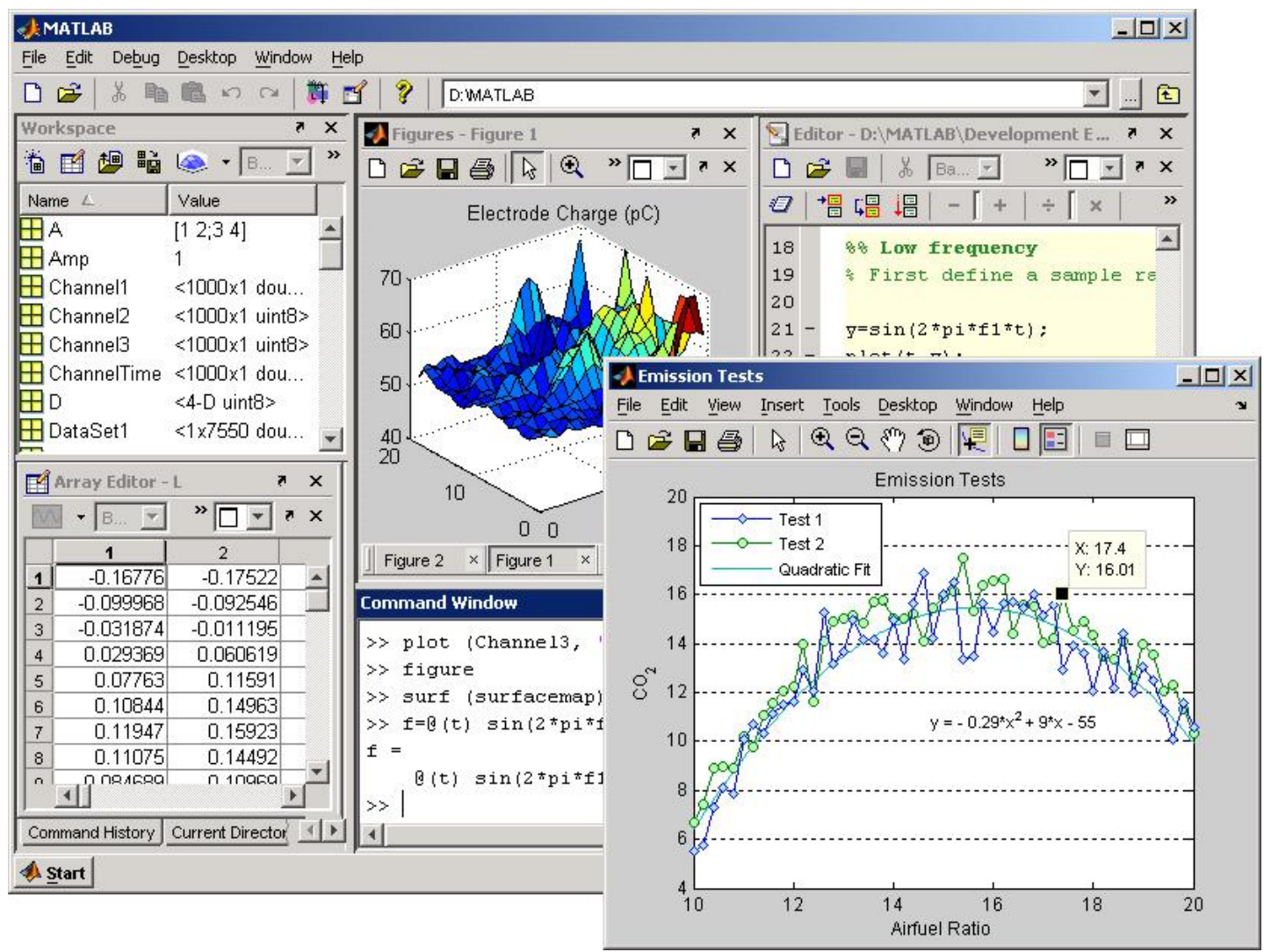

Figure 3- 2: Typical MATLAB desktop view

As mentioned earlier MATLAB is a fourth-generation programming language which is an object oriented programming. MATLAB is widely used in engineering analysis because of its user friendly programming nature. Most basic programming aspects like variable declaration, memory allocation, and data type specification are default. MATLAB supports structure array data types. It supports classes; however the syntax and calling conventions are noticeably different from other programming languages. Computation is considerably fast because of its matrix solving methodology. It supports excellent GUI for computing using graphical symbols of the devices/equipments/objects. It uses MuPAD symbolic engine, allowing access to symbolic 
computing capabilities. Below mentioned are few attributes of MATLAB which makes it an excellent engineering analysis tool [40]:

- Technical computing adopting high level language

- Easily manageable code, files and data

- Wide range of toolboxes supporting iterative investigation and problem solving

- Mathematical functions for linear algebra, statistics, Fourier analysis, filtering, optimization, and numerical integration

- Excellent graphic support (2-D and 3-D) for data visualization

- Supports development of custom build GUI

MATLAB is also used for power system analysis. Various packages that support analyses of power system using MATLAB are developed and widely utilized. A SimPower system is a Simulink toolbox provided by MATLAB to support power system analysis. Apart from toolboxes provided by MATLAB, there are various packages like MATPOWER, PSAT, and PAT etc were developed. However, due the complex nature of power distribution system (PDS), MATLAB may not be a good choice of large scale power distribution system or for transient analysis on PDS.

In the present thesis work MATLAB is used to forecast electricity load on the system using neural networks toolbox provided.

\subsection{Java Agent DEvelopment framework}

Java Agent DEvelopment framework (JADE) is Java based software package to support multi- 
agent system (MAS) implementation. It facilitates development of MAS with middle ware that complies with Foundation for Intelligent Physical Agents (FIPA). It also provides tool box to support debugging and deployment phases of MAS development. Since JADE is implemented in Java it needs Java run time environment for its execution. Agents can be executed in different machine not necessarily sharing the operating systems. Agent can be operated, changed and moved during run time in remote GUI. Features like Object Serialization, Reflection API and Remote Method Invocation (RMI) are available with JADE since it is programmed using OOP's based JAVA.

Developed by Telecom Italia, JADE is an open source code under the terms of the LGPL (Lesser General Public License Version 2). JADE complies to FIPA specifications and has an excellent agent management model which includes 1) Agent Management systems, 2) Directory Facilitator, and 3) Message transport system along with any number of agents which may be distributed on different machines. JADE is widely used agent-oriented middleware today.

\subsubsection{JADE Platform and Architecture}

JADE is composed of various following mentioned packages which makes it complied with FIPA specifications [43] [4].

- jade.core is the main package which implements the kernel of the system, it also includes Agent class and Behaviour class is included in sub package jade.core.behaviours which basically indicated the intention of agent acts

- jade.lang.acl sub-package provides agent communication language process according to FIPA specifications 
- jade.content package includes content to support user defined Ontologies and content-languages

- jade.domain includes classes of Java that represents the agent management entities as specified by FIPA. Those include Agent Management System (AMS) and Directory Facilitator (DF) which provide life cycle, white page and yellow page services

- $\quad$ jade.gui contains set of classes which is useful to create GUI to display and edit Agent-Identifies

- $\quad$ jade.mtp included Java interface classes which are needed by every Message Transport Protocol (MTP) to be able to integrate with the JADE framework

- jade.proto package includes those classes which can enable standard interaction protocol needed for agents to communicate

- jade.wrapper packages which are useful when external Java applications launch JADE agents and containers. It provides wrappers of JADe hih-level applications

- jade.tools package provides bundle of tools to assist administration and simply application development. Remote Management Agent, Dummy Agent, Sniffer Agent and DF GUI are few of them 


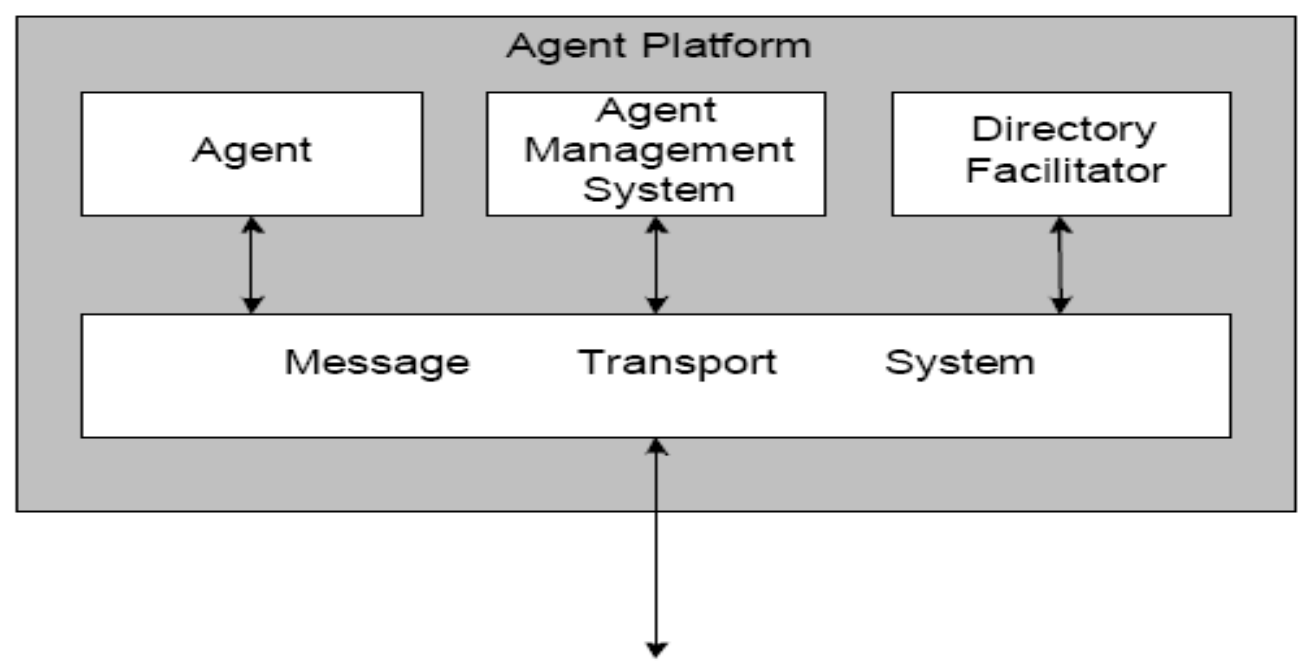

Figure 3- 3: Reference architecture of a JADE agent [43]

Figure 3-3 represents reference architecture of a FIPA specifies agent which JADE is complied to. AMS is an agent which has supervisory control over access to and use of agent platform. DF provides default yellow page services to the agents in the platform. The Message Transport System also called as Agent Communication Channel (ACC) is a software component whose basic functionality is to control the exchange of messages. AMS and DF are immediately created as soon as JADE platform is launched complying with FIPA standards. 


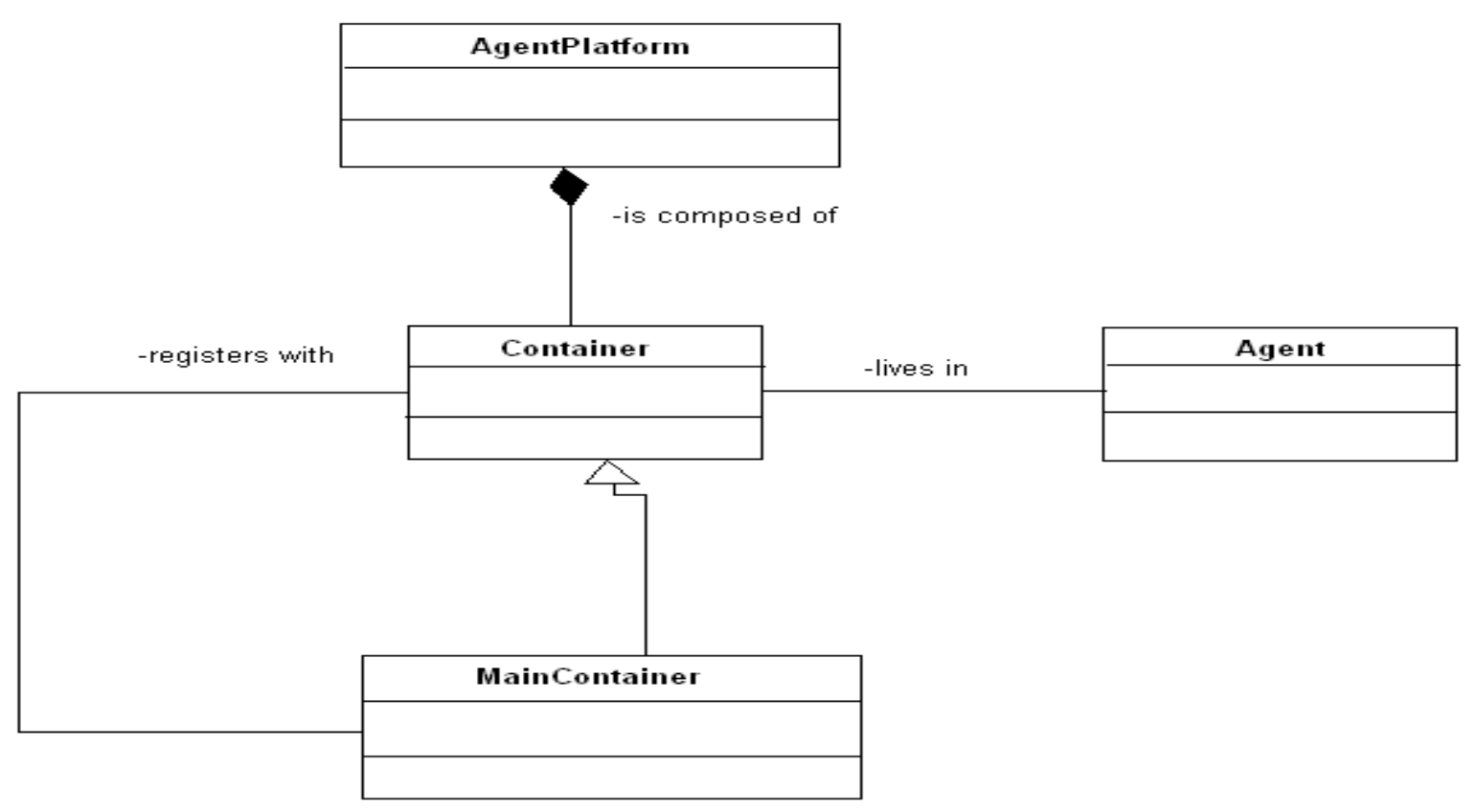

Figure 3- 4: Main architectural elements and relationship between them [4]

Figure 3-4 depicts main architectural elements of JADE platform. Containers are Java process which provides necessary environment for agents to be hosted and executed. Agents live in containers. Main container is a special container where DF and AMS agents will be initiated as soon as the JADE platform is launched. Main container is the first container to be launched and later all the rest container have to register with the main container. As a bootstrap point, main container has following responsibilities:

- Managing the container table. Object references and transport addresses of all containers is maintained in container table

- Managing the Global Agent Descriptor table (GADT). Agent identity, status and location is registered in GADT

- Hosting AMS and DF. As mentioned earlier there two special agents provide white page and yellow page services to the platform 


\subsubsection{JADE Programming}

As mentioned in earlier sections JADE is Java based programming package with full Java tools, so creating a JADE-based multi-agent system only implies creating Java classes without any considerable expertise in Java programming.

To create an agent all that one needs to do is to implement setup () method in a class which extends jade.core. Agent class included in jade.core package. The setup () method is intended for agent initialization. Typical operations performed by an agent in its setup () method include a)showing a GUI, b)opening a connection to a database, c) registering the services with yellow pages, and d) starting the initial behaviors [4].

Instance of the class jade.core. AID is used to represent agent identifiers in JADE. To retrieve the agent identity getAID () method from Agent class is used. The agent name in JADE has the format <local-name>@<platform-name>. An AID object includes globally unique name and address. Normal Java classes can be used to compile JADE classes provided JADE libraries are in the classpath. After the agent has done what it is supposed to be doing, it still stays alive by default. doDelete() method must be called to terminate it and takedown () method must be invoked before it to do some clean up. Agent can accept start up arguments and the arguments thus passed can be retrieved by means of getArguments () method.

Behaviours are those which decide what tasks agent will be doing. These are implemented as objects of class jade.core.behaviours.Behaviour. Adding a behaviour using 
addBehaviour() method of the Agent class will enable agent to execute the job implemented by a behaviour object. Every class extending Behaviour must implement two abstract methods called action () and done (). The action () method defines the operation and done ( ) method indicates whether the behaviour is completed or not. Depending on type of task to be implemented there are different classes of behaviour namely One-Shot Behaviours, Cyclic Behaviours and Generic Behaviours.

\subsection{IBM ILOG CPLEX Optimization Studio}

IBM ILOG CPLEX is an optimization software package [45]. This advanced mathematical programming technology has robust algorithm for demanding problems, which can solve optimization problems with millions of constraints. The software can solve large real-time system optimization problems. It has a GUI and can also be interface with various programming languages like $\mathrm{C}, \mathrm{C}++, \mathrm{C} \#$ and Java. In the present thesis work it has been integrated with JADE for optimized load management.

\subsection{Conclusions}

This chapter briefly elucidates the software packages used in this thesis work to solve the problem. MATLAB is used to forecast the load. With MATLAB toolboxes a excel standalone Load Forecaster has been implemented. JADE is used to model the multi-agent system which manages the load and distributed generations in order to reduce peak demand. Agents developed in JADE will make use of forecasted load outputted as excel sheet using MATLAB. 


\section{Chapter 4}

\section{SIMULATIONS AND RESULTS}

In this chapter, load management algorithm and its simulation are elucidated. Multi-Agent Systems (MAS) has been developed in JADE and its deployment is discussed in this chapter. MAS form a load management module which is employed to reduce the peak electricity demand by 15 percent. MATLAB is used to develop short-term load forecasting tool.

\subsection{Load Forecaster}

MATLAB is employed to develop load forecaster. Historical hourly load data, dew point, dry bulb, and seasonality (holiday, hour, and weekday) are used to calibrate the load forecaster. Once the forecaster is deployed, forecasted temperature, day and type of the day is given as an input to the forecaster to forecast the day ahead load. The load forecaster implemented in the present thesis work is motivated by Electricity Load and Price Forecasting case study implemented by Math Works.

\subsubsection{Database for Load Forecasting}

Historical hourly load data, historical hourly dew point, historical hourly dry bulb data and type of the day data is used as the database to develop load forecaster. One year system load data obtained from test distribution feeders West Run $1 \& 2$ are used as base database. The database 
connection is made using Microsoft access database files.

The Database toolbox from the MATLAB is used to set up the query with required data variables obtained from Microsoft access database files. This toolbox helps to use MATLAB functions to import and export data between the MATLAB workspace and relational databases [44]. Figure 4-1 shows Visual Query Builder (VQB) interface from Database toolbox provided by MATLAB. VQB is used to import the data from test feeder database to MATLAB, where load forecasting is developed.

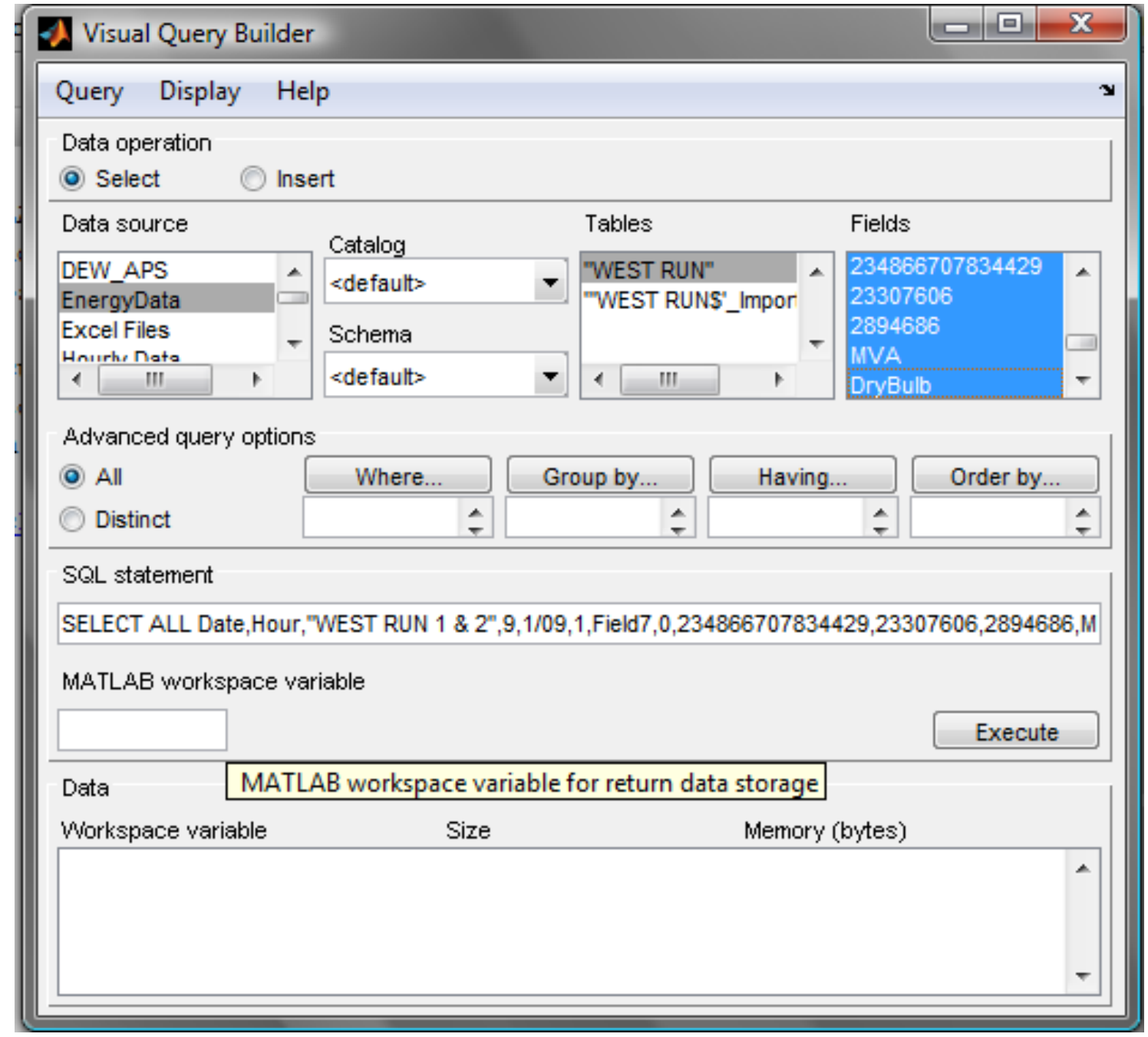

Figure 4- 1: Visual query builder interface from Database toolbox 


\subsubsection{Linear regression}

Linear regression is the traditional statistical approach used to design or model the relationship between a scalar variable $\mathrm{y}$ and one or more variables $\mathrm{X}$. In other words, linear regression is used in this thesis work to fit a load predictive model to an observed data set of system load (y) and observed historical values of temperature, date, type of the day, day of the week etc. (X). After such load forecast model has been developed, if an additional value of $\mathrm{X}$ is given without its accompanying value of $y$, the fitted model can be used to make a prediction of the value of $y$.

Statistics toolbox provided by MATLAB is used to implement the forecast model. The toolbox has two categories of tools which can be used in coding and in GUI. The function regress $(y, X)$ returns a $p$-by- 1 vector $\mathrm{b}$ of coefficient estimates for a multi-linear regression of the responses in $\mathrm{y}$ on the predictors in $\mathrm{X}$. X is an $n$-by- $p$ matrix of $p$ predictors at each of $n$ observations. y is an $n$ by-1 vector of observed responses. Linear regression method from statistics toolbox is applied as follows:

$[\mathrm{b}, \mathrm{bint}, \mathrm{r}]=\operatorname{regress}(\mathrm{y}, \mathrm{x})$;

where, bint is the coefficient estimates interval and $r$ is residuals.

yPred $=\mathrm{X} * \mathrm{~b} ;$ where, yPred is the predicted system load.

Figure 4- 2 shows the prediction of system load and accuracy of the prediction. It shows that there is a considerable difference in the predicted values and actually value. Hence, multipleregression technique or neural network training approach can be used for reduction in the error. 


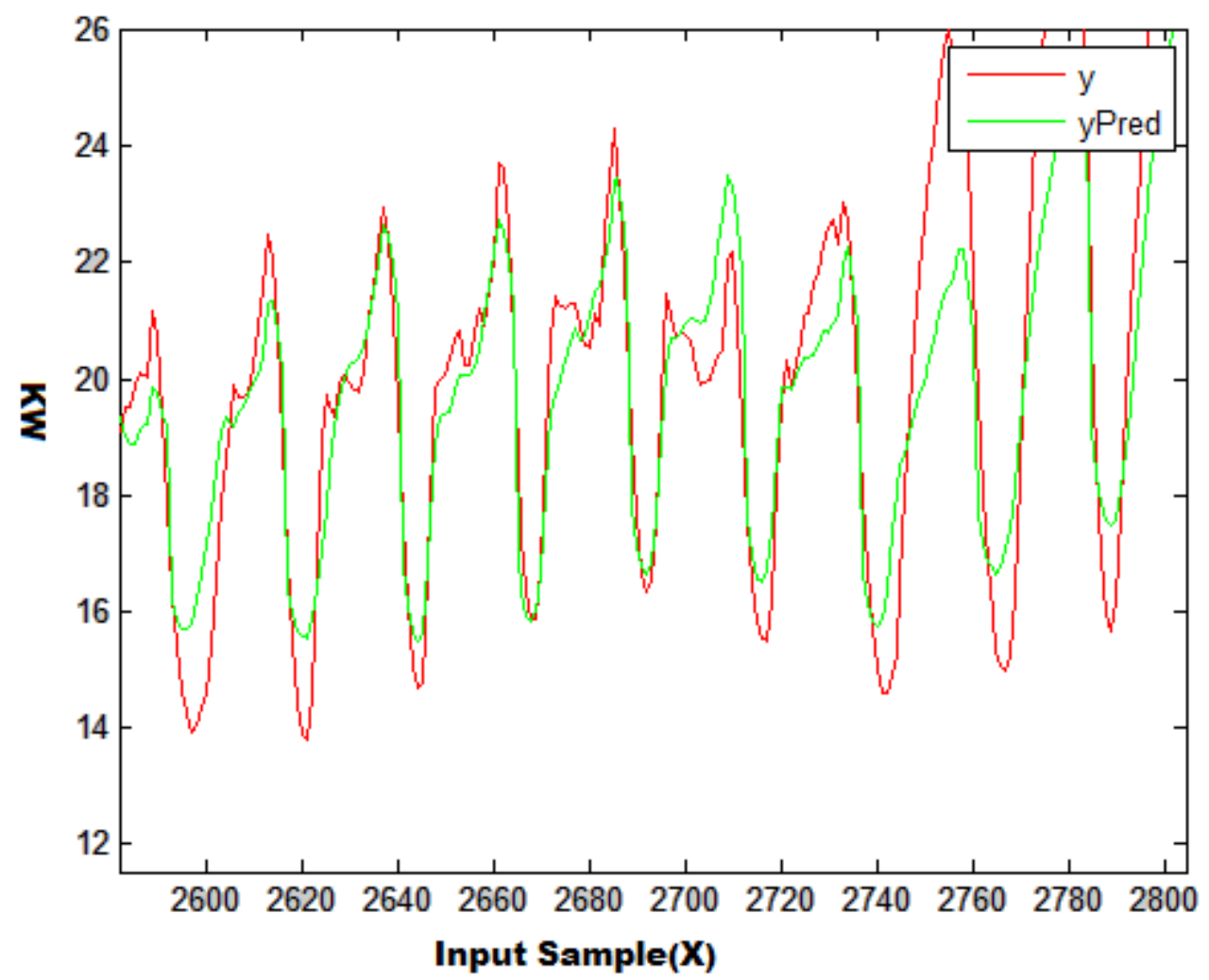

Figure 4- 2: Prediction of accuracy with linear regression

\subsubsection{Neural Network}

Neural networks are composed of simple elements which are similar to biological nervous system elements operating in parallel. The elements can be trained to perform particular task ranging from pattern recognition, identification, classification, speech, vision, and control systems. Neural network elements are trained by adjusting the connections called as fitting between elements as shown in the following block diagram: 


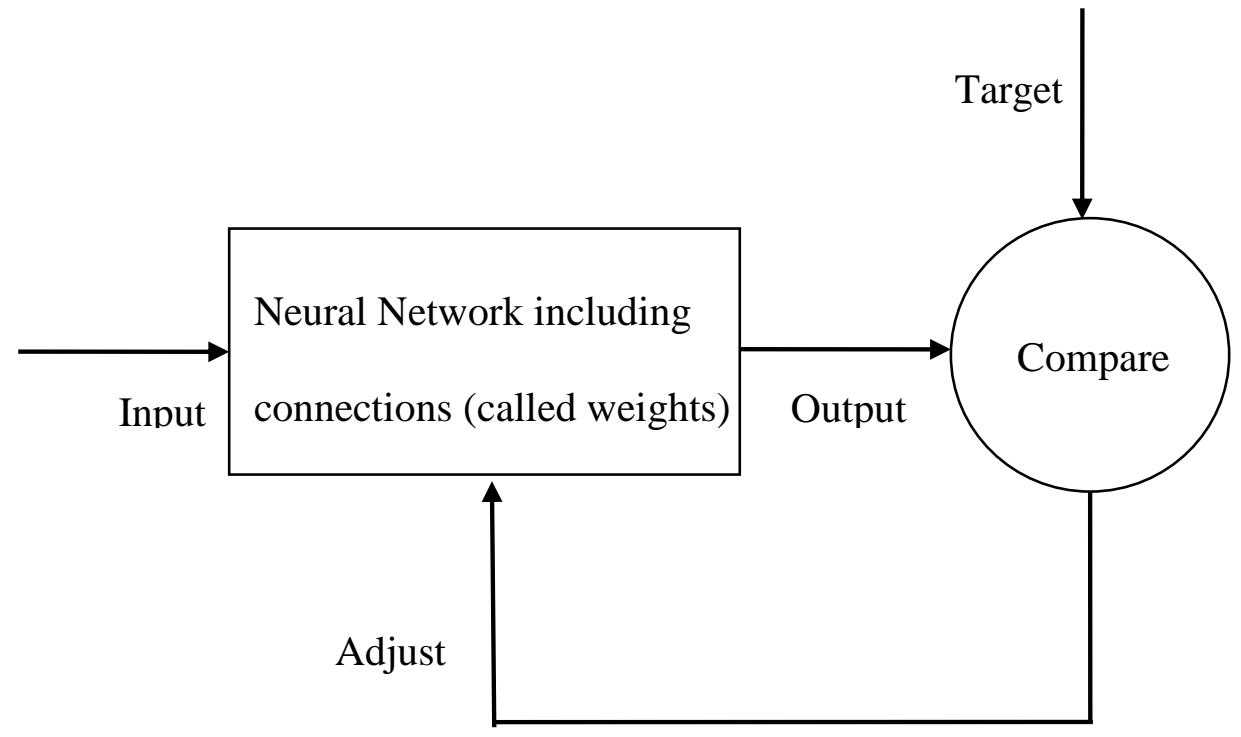

Figure 4- 3: Neural network training block diagram

Neural network toolbox provided by MATLAB is used to implement the load forecast model in this thesis work. The Neural Network Fitting Tool will help to select data, create and train a network, and evaluate its performance using mean square error and regression analysis with a two layer feed forward neural network. Neural network fitting tool is used to perform the load forecast task where the elements are trained using Levenberg-Marquardt back propagation algorithm.

In the process of forecasting the load using neural network toolbox, the following steps are performed,

- Selecting data: Inputs and targets are defined initially. In this present work, 8760 samples of 8 elements (dry bulb, dew point, hour, weekday, is working day, previous day same hour load and previous 24 hour average load) are given has input and 8760 samples of 1 element (system load) is given as target. 
- Validation and testing data: Validation and testing of the neural network elements is an important criterion in the load forecasting process. 6132 samples are used for training purpose, 1314 for validation and 1314 for testing. Training samples are presented during the training process and the network error is adjusted accordingly. Validation samples are used to measure the network generalization. Testing samples provide network performance during and after training.

- Network size: Network size determines number of neurons in the hidden layer. To improve the performance of the network, number of neurons in the hidden layer can be increased. Figure 4-4 represents the neural network layers adapted in this present thesis work.

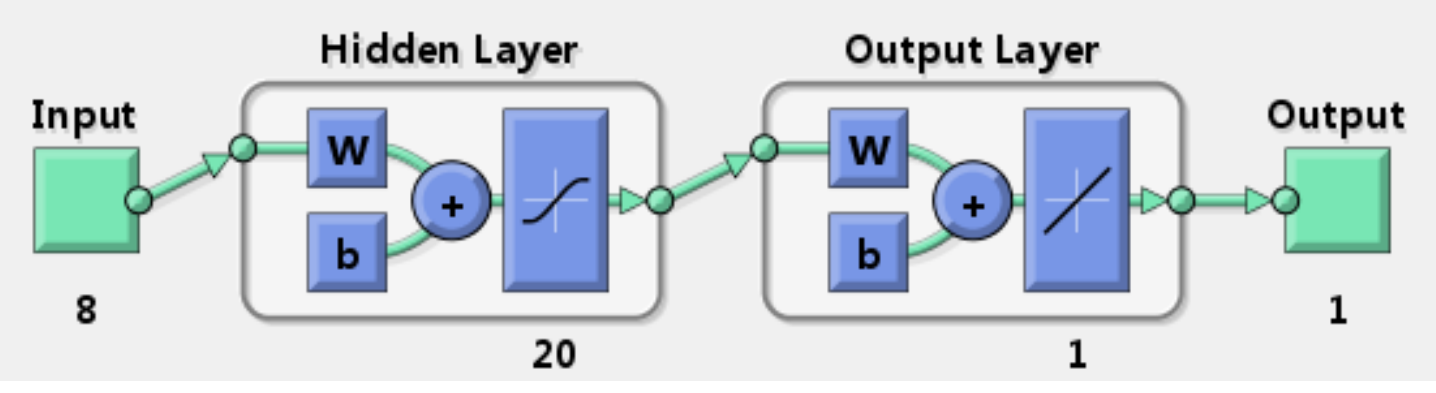

Figure 4- 4: Neural network layers with number of neurons

- Training Network: Network is trained to fit inputs and targets. In the present thesis work default Levenberg-Marquardt back propagation algorithm is used for training. Mean squared error (MSE) is average squared between outputs and targets. Lower value of MSE is better. Regression value shows the correlation between outputs and targets ( 1 representing perfect match and 0 representing no match). Figure 4-5 shows the training results of neural networks with available data. 


\begin{tabular}{llcc} 
Results & & & \\
& Samples & & \\
Training: & 6132 & $1.78457 \mathrm{e}-0$ & $9.56801 \mathrm{e}-1$ \\
Validation: & 1314 & $2.31341 \mathrm{e}-0$ & $9.45417 \mathrm{e}-1$ \\
Testing: & 1314 & $2.41483 \mathrm{e}-0$ & $9.47609 \mathrm{e}-1$ \\
\hline
\end{tabular}

Figure 4- 5: System load forecast results using neural network toolbox

Figure 4-6 represents training regression plot, Figure 4-7 represents validation regression plot, and Figure 4-8 represents test regression plot with regression value ' $R$ ' $0.9568,0.94542$, and 0.94761 .

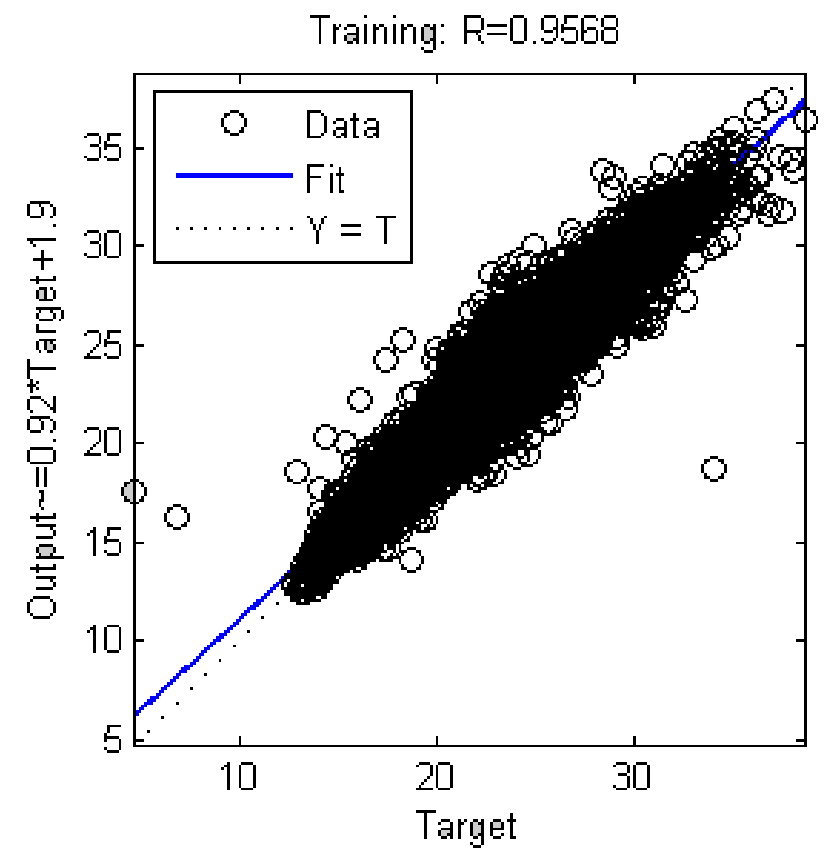

Figure 4- 6: Neural network regression plot during training 


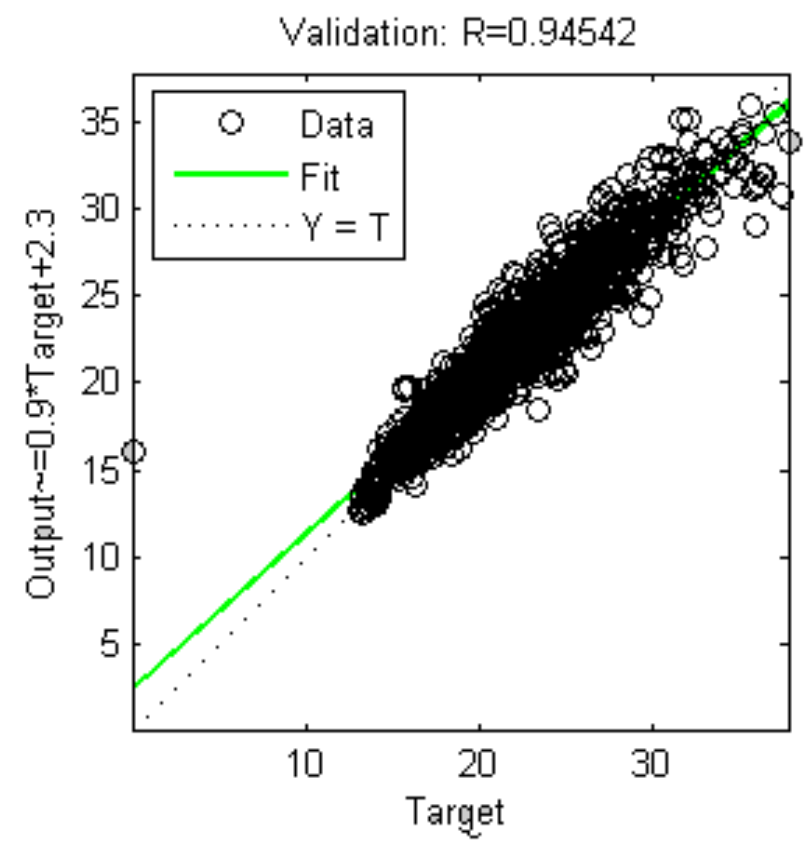

Figure 4- 7: Neural network regression plot during validation

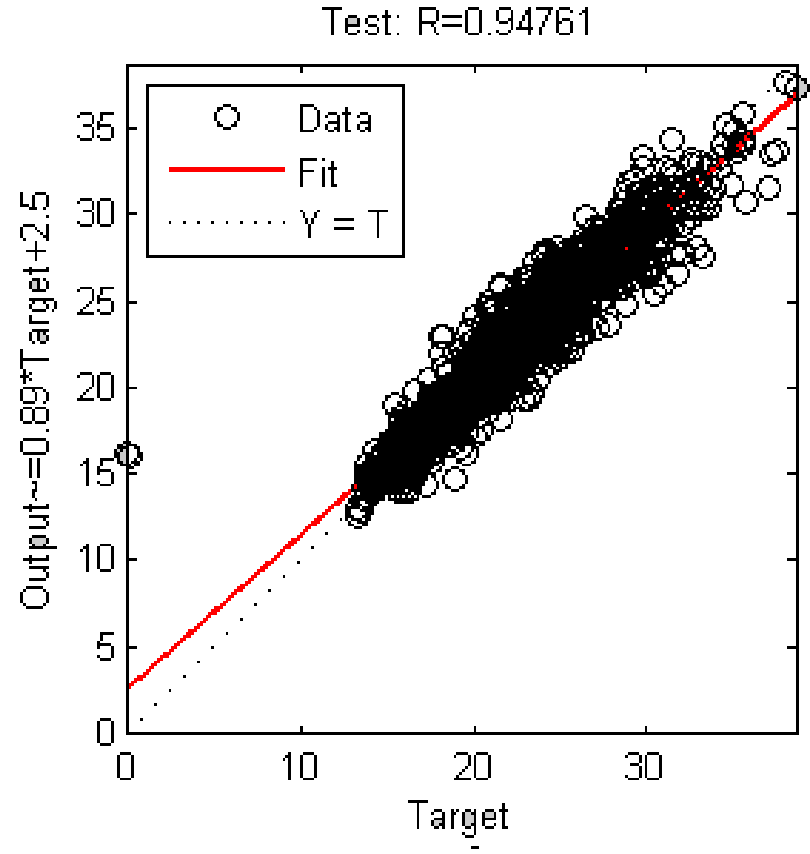

Figure 4- 8: Neural network regression plot during validation 
Figure 4-9 represents MATLAB neural networks training GUI.

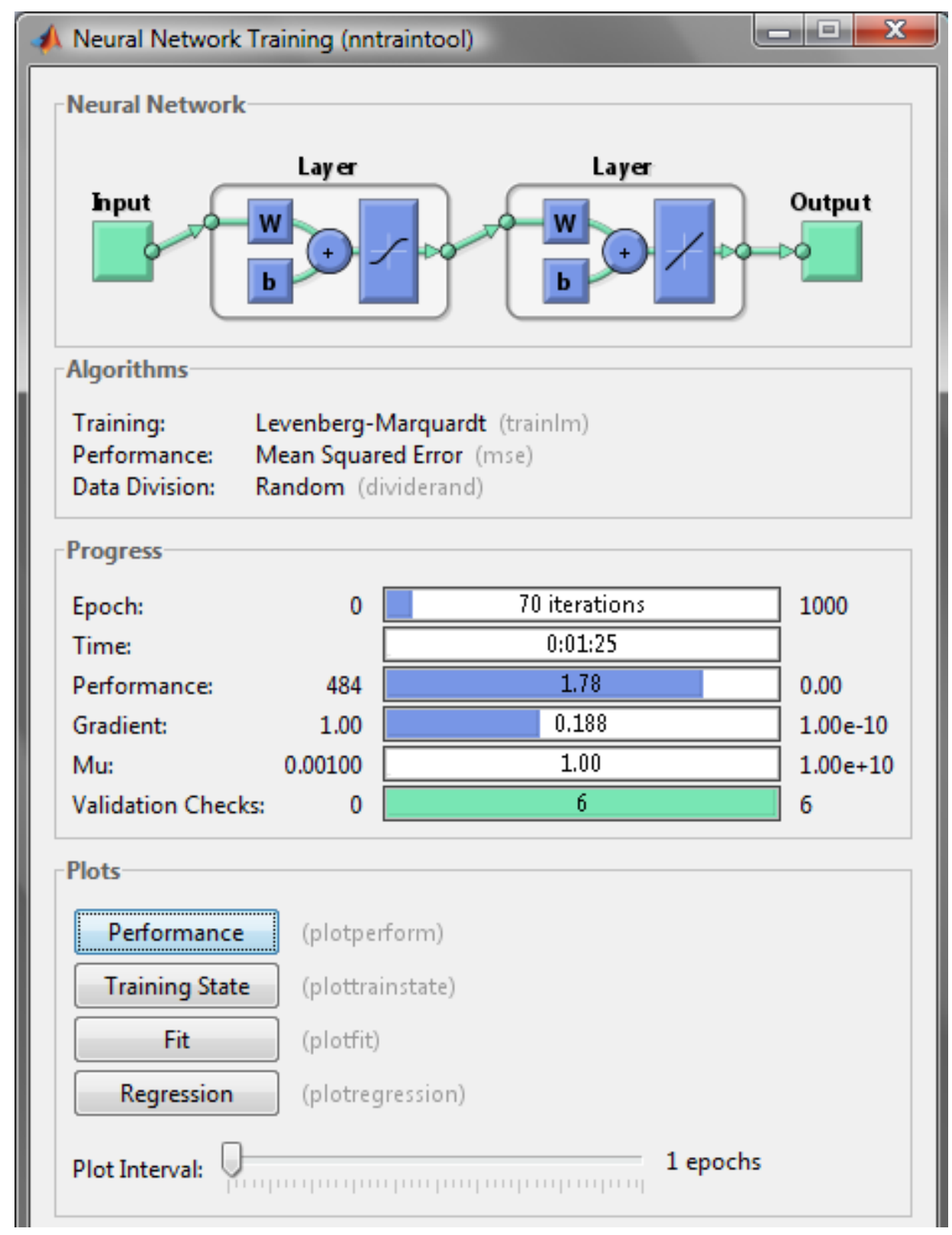

Figure 4- 9: Snapshot of neural networks training tool interface

\subsubsection{Load Forecasting with Neural Network Prediction}

Load forecasting is performed with the pre-trained neural networks. Date, temperature, and whether the day is a holiday or not information are given as an input to forecast the load. The below pseudo code explains how the load forecast is obtained. 
The following pseudo code is employed to process input data

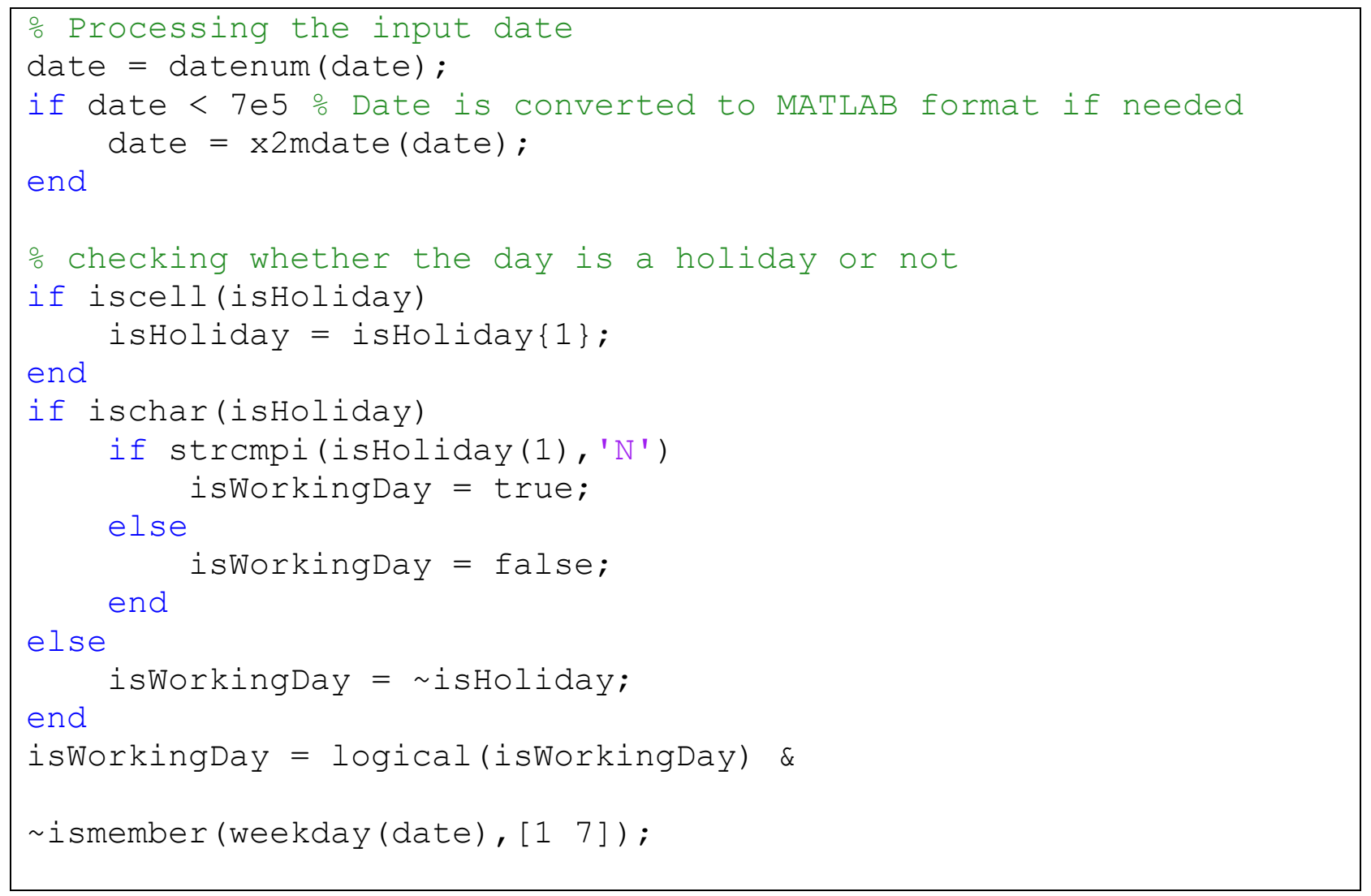

The following pseudo code is employed to process input data for further calibration:

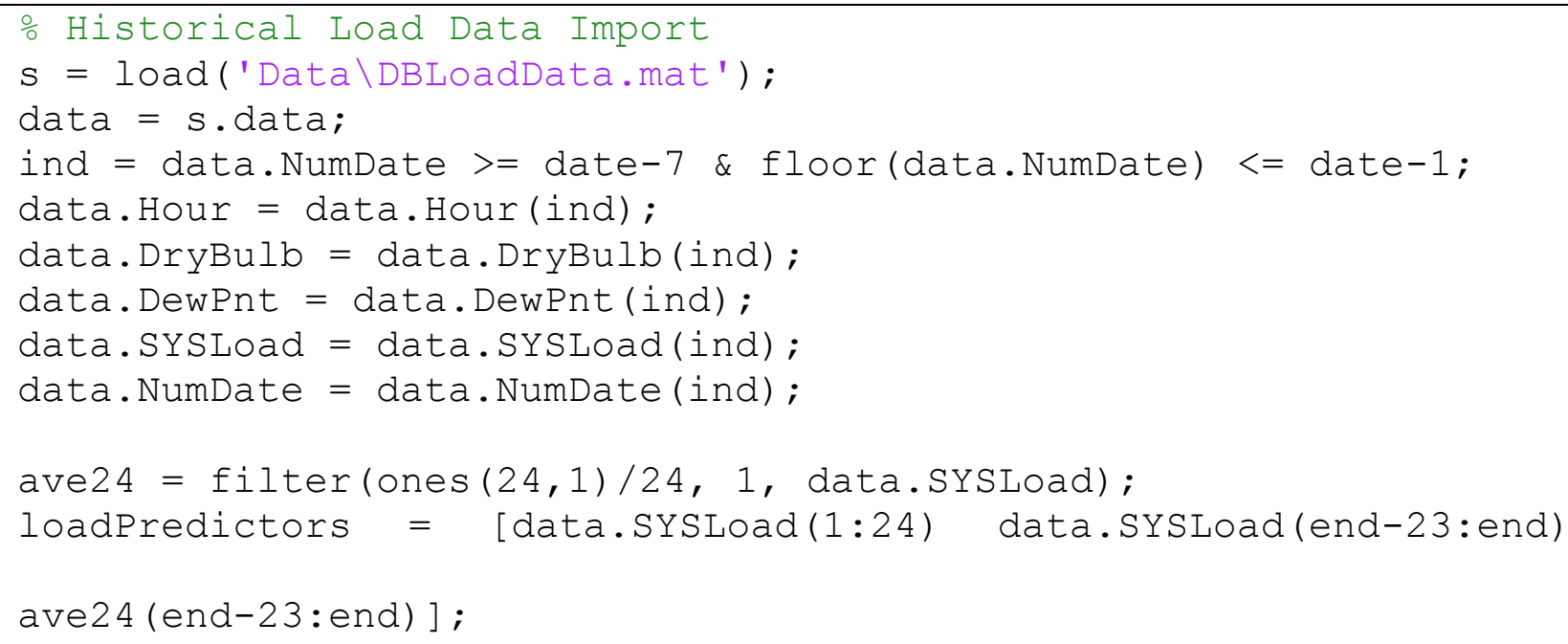


The data obtained from the West Run feeder is imported to the MATLAB and eight predictors are allocated for load prediction. Below code creates a load prediction matrix X.

$\mathrm{X} \quad$ [temperature $(1: 24,1: 3)$
isWorkingDay*ones $(24,1)$ loadPredictors];

After the neural networks are trained as explained in section 4.2.3, the trained model is saved as a MATLAB data file and it is accessed to predict the load as shown below.
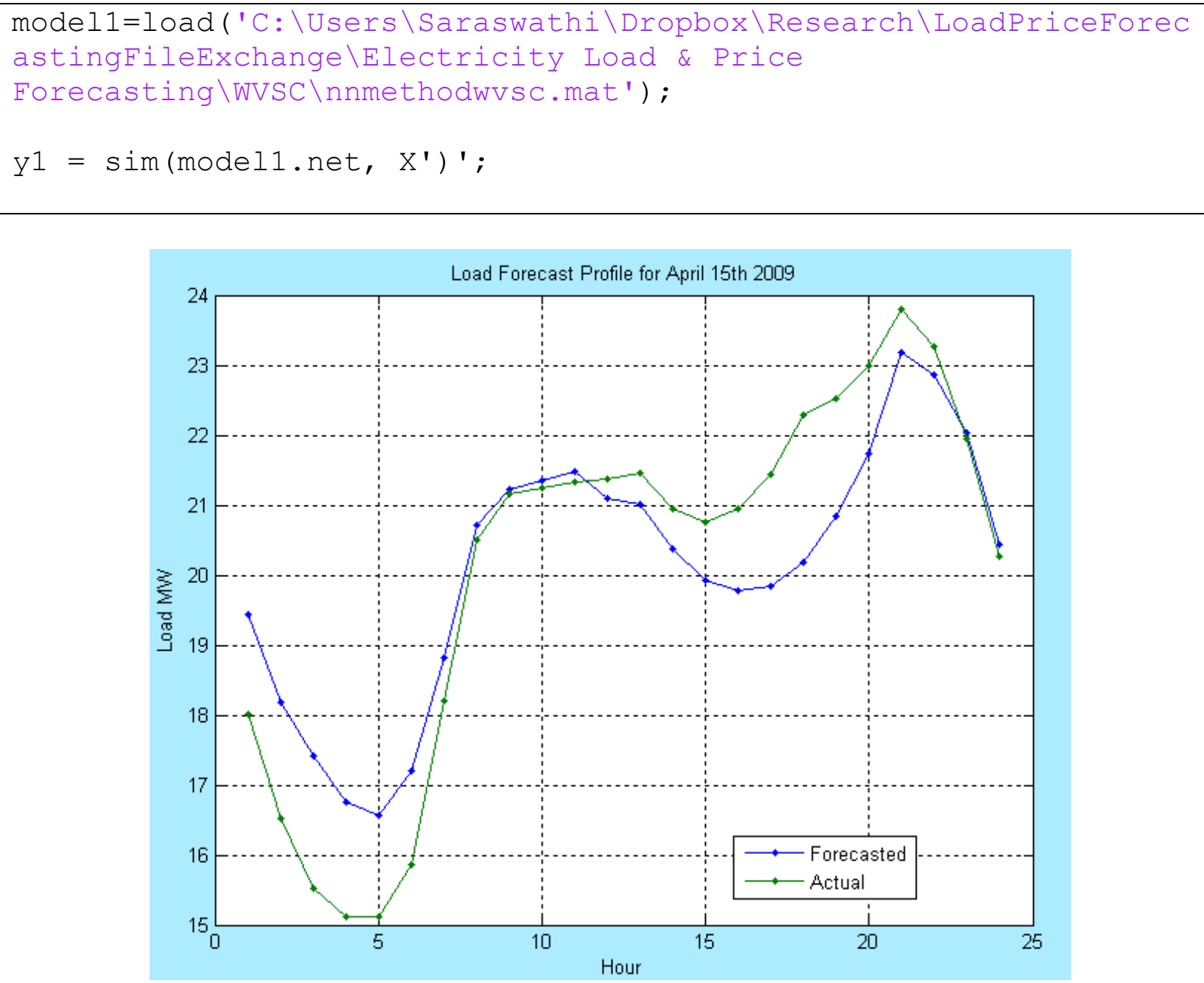

Figure 4- 10: Load Forecast Predicted by Neural Network Model and Actual Load 
Figure 4-10 depicts typical system load on West Run feeder during April $15^{\text {th }}$ of 2009 as predicted by neural network models and actual load on the same date. Figure 4-11 represents system load during September $1^{\text {st }}$ of 2009 . Both the figures predict the peak demand occurring time and peak demand level. The predicted system load is exported to an excel sheet. The forecasted load information is used by Load Management System (LMS) for peak demand reduction by 15 percent.

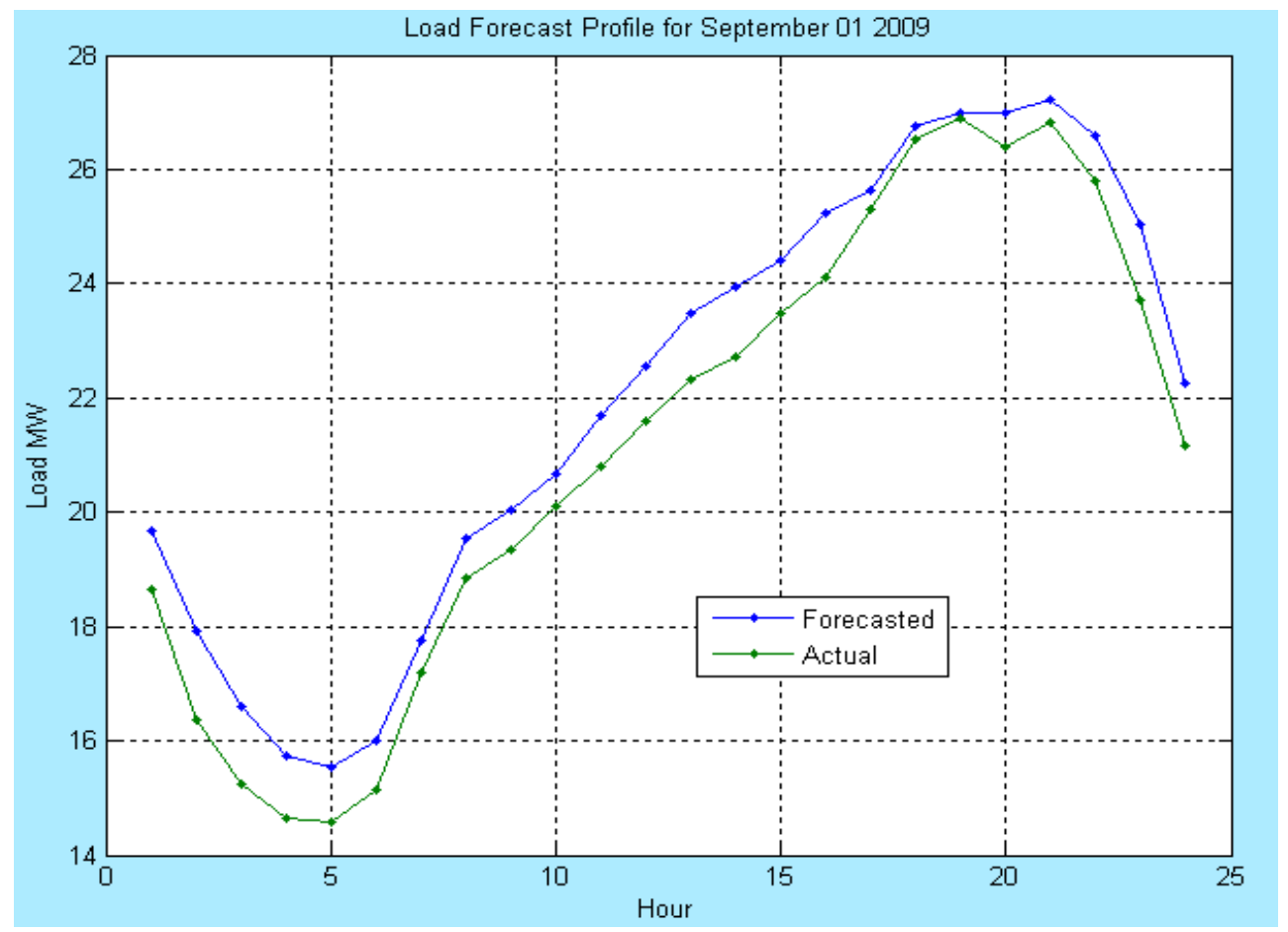

Figure 4- 11: Load Forecast Predicted by Neural Network Model and Actual Load

\subsubsection{Model Comparisons}

In this present thesis work the load forecaster is developed using linear regression technique (Statistics Toolbox) and Artificial neural networks (Neural networks toolbox). It has been observed that the Mean Absolute Percentage Error (MAPE) for the prediction using linear regression (7.283) is more compared to that of neural networks (6.120). 


\subsection{MAS Architecture}

The proposed MAS have hierarchy of agents at different layers as depicted in Figure 4-12.

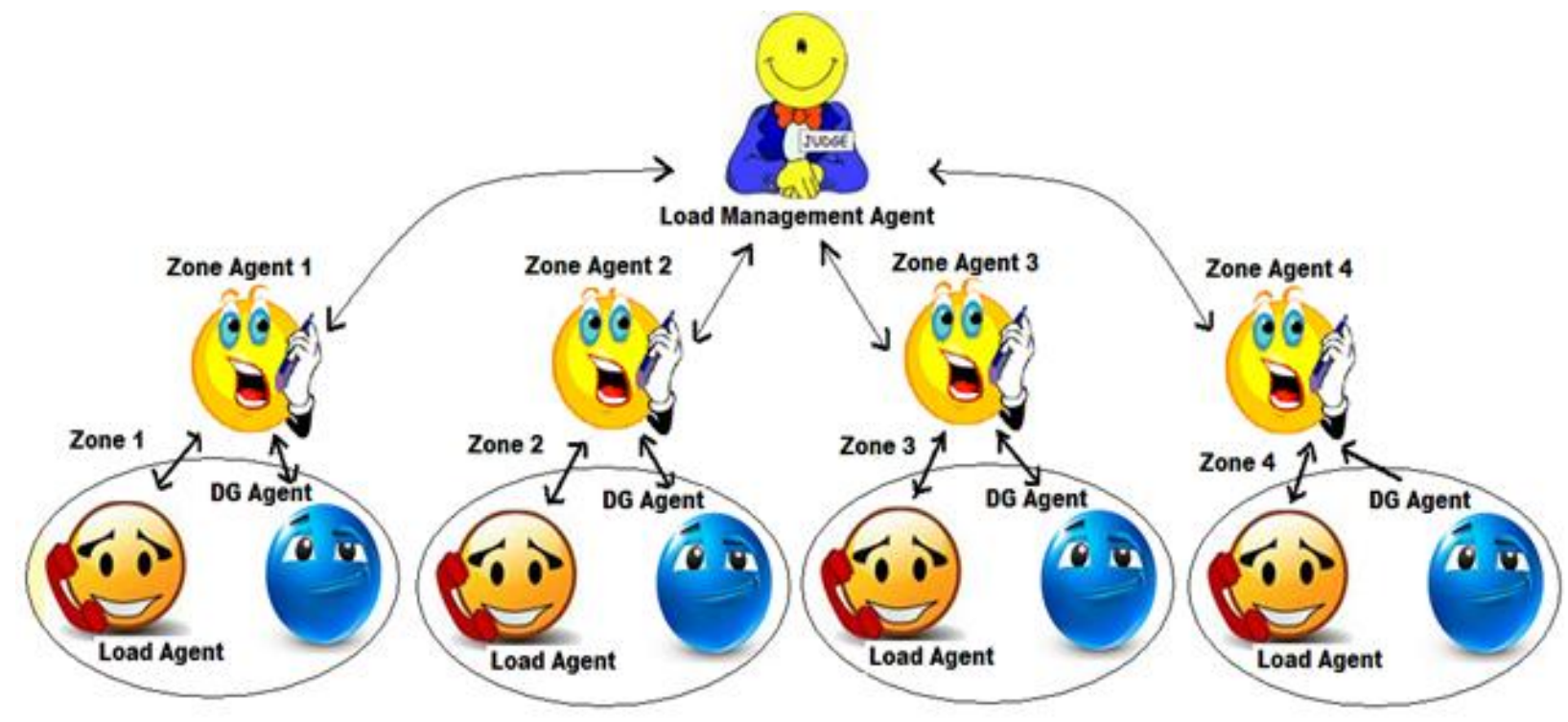

Figure 4- 12: Proposed MAS architecture

The service area is assumed to be divided into four zones with one agent pertaining to each zone. Zone Agent (ZA) has the capability to communicate with Load Management Agent (LMA) and any agents of that particular zone. Every zone has one Load Aggregator Agent (LAA) and a Distributed Generation Agent (DGA) associated with respective load and DG. The Load Management System (LMS) proposed in this thesis uses a centralized approach. Responsibilities and communication capabilities of each agent are discussed below,

- Load Management Agent (LMA): LMA is the head agent of the load management system proposed in this thesis. It is the decision taking agent. LMA takes the day ahead forecasted load predicted by the load forecaster and identifies the peak demand level, limit, and time in which peak demand is occurring. Accordingly LMA requests controllable loads availability and DG availability from zone agents. When LMA 
receives all needed information from ZAs controllable load and DG operation time are allocated. LMA is majorly responsible for peak load reduction allocation and can communicate only with zone agents so as to reduce the communication traffic.

- Zone Agent (ZA): ZA has the information about DG and controllable loads available in its respective zone. Zone agent is the only agent which can communicate with LMA, DGA and LAA. As soon as ZA receives the request from LMA, ZA in-turn sends request to DGA and LAA to check KW capability and controllable load availability respectively. After the necessary information is received ZA sends the details to the LMA.

- Load Aggregator Agent (LAA): LAA has the information about controllable load availability, charging rate, and required $\mathrm{KWH}$. There can be any number of LAA in a given zone, but only one LAA is considered in each zone. Requirements of all the all controllable loads are aggregated and the information is fed to LAA. LAA communicates only with ZA.

- Distributed Generation Agent (DGA): DGA has information about the DG to which it is associated. Ideally it also has DG Kilo Watt (KW) capacity and time from which the DG can be available. Minimum start up time required for the DG to be connected to grid is assumed to be included in the time from which DG can be available.

Figure 4-13 shows the possible location of MAS on the physical layer of power distribution feeder (West Run) considered in this thesis work 


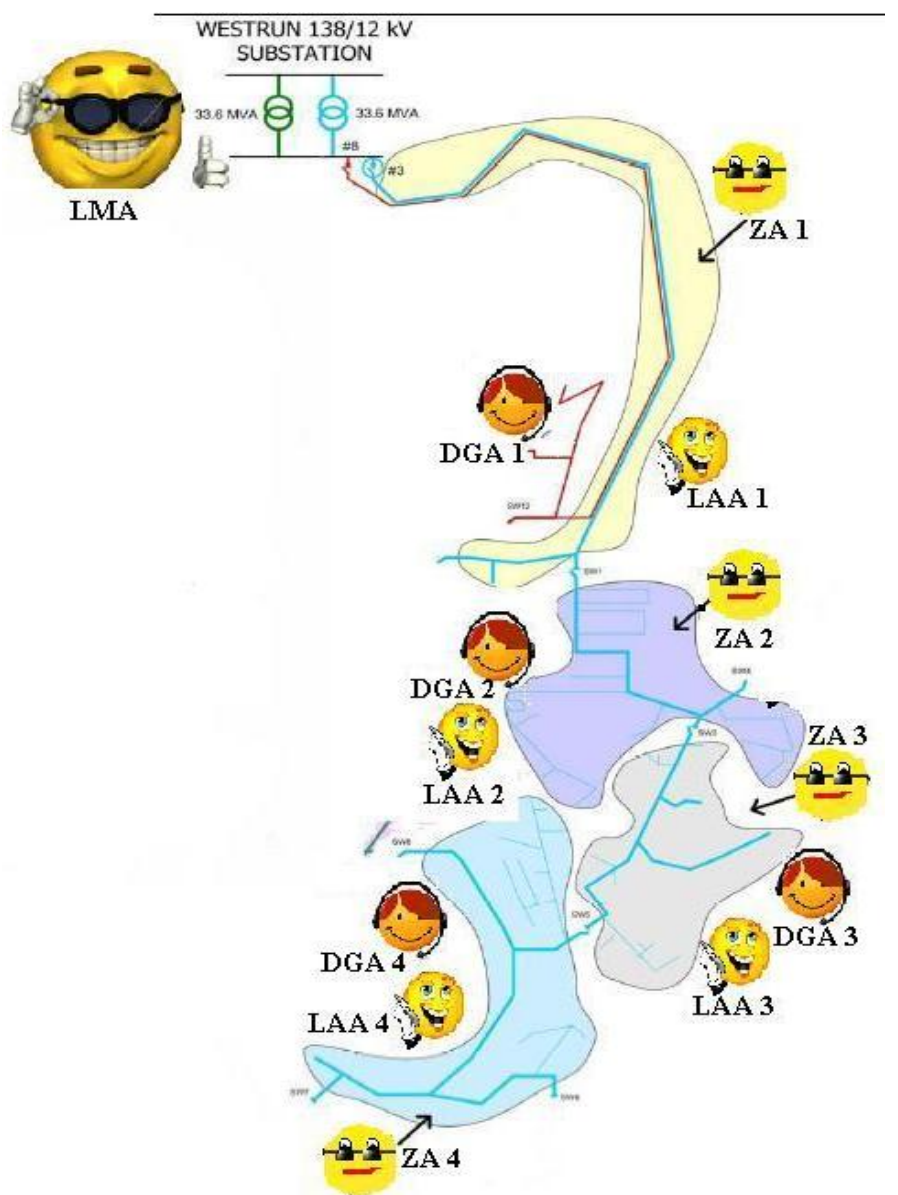

Figure 4- 13: Depiction of agent's location on West Run Feeder

\subsection{Load Management System}

The proposed load management algorithm includes electric load forecasting using MATLAB and peak demand reduction using MAS. Figure 4-14 shows the inputs needed to calibrate the MATLAB load forecaster, inputs needed to forecast the load, and the output. 


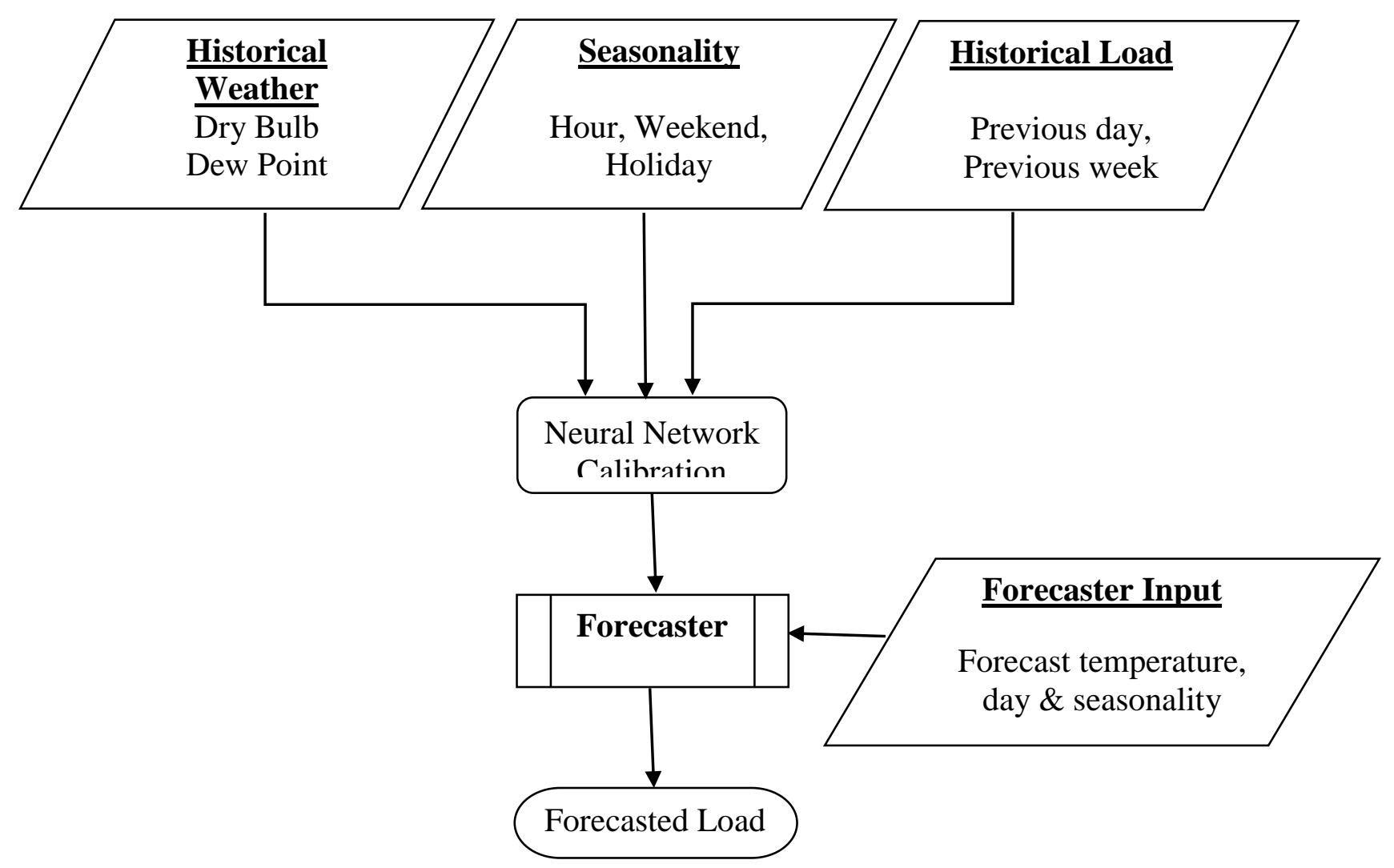

Figure 4- 14: Load Forecasting Flowchart

As elucidated in the section 4.1, electric load has been forecasted and the forecast for the specified inputs is shown in the Table 4-1. Peak load reduction is handled by MAS, developed using JADE. The forecasted load is given as an input to MAS.

Peak demand reduction using MAS is performed by adopting two strategies. First strategy considers no price signal but assumes that rewarding DGs and Loads for their participation in the load management is taken care of. In the second strategy price signal as bid sent by DGs and Loads is considered. And it is assumed that DGs and Loads are available during the peak time and load management is done in the cheapest way possible using optimization technique. 
Table 4- 1: Load forecast output from MATLAB

\begin{tabular}{|c|c|c|c|}
\hline Hour & Dry Bulb & Dew Point & Forecast(MW) \\
\hline 1 & 38 & 31 & 21.2208 \\
\hline 2 & 36 & 30 & 20.1878 \\
\hline 3 & 36 & 30 & 19.3578 \\
\hline 4 & 36 & 30 & 18.6598 \\
\hline 5 & 35 & 30 & 18.3294 \\
\hline 6 & 34 & 29 & 18.6346 \\
\hline 7 & 35 & 30 & 19.4703 \\
\hline 8 & 35 & 31 & 20.4247 \\
\hline 9 & 36 & 32 & 20.9876 \\
\hline 10 & 38 & 32 & 21.7635 \\
\hline 11 & 40 & 32 & 22.5264 \\
\hline 12 & 41 & 32 & 22.9449 \\
\hline 13 & 42 & 32 & 23.3009 \\
\hline 14 & 43 & 32 & 23.1674 \\
\hline 15 & 44 & 32 & 23.0896 \\
\hline 16 & 44 & 32 & 22.9185 \\
\hline 17 & 44 & 34 & 22.3871 \\
\hline 18 & 43 & 35 & 22.0426 \\
\hline 19 & 42 & 36 & 21.4414 \\
\hline 20 & 41 & 37 & 21.2354 \\
\hline 21 & 40 & 38 & 20.7774 \\
\hline 22 & 40 & 38 & 19.8053 \\
\hline 23 & 40 & 38 & 18.7465 \\
\hline 24 & 40 & 38 & 17.5666 \\
\hline & & & \\
\hline
\end{tabular}

The proposed MAS have 13 agents located in different layers. With respective to the devices with which agents are associated, various data has been given as an input to all the agents for the purpose of simulation. Table 4-2 shows typical data given to respective agents. This data can be related to the real time zone, DG, and load data. LMA however has all the data needed for it to dispatch the load for the purpose of peak demand reduction. LAA has the data of all the controllable loads aggregated. DGA has the DG characteristics, its availability, capacity and bid. Once the LMA identifies the peak demand constraints (Peak demand level, occurring time, and cut-off limit), it initiates the communication process for the peak reduction. 
Table 4- 2: (a) Load Management Agent (LMA) Data, (b) Zone Agent (ZA) Data, (c) Distributed Generation Agent (DGA), and (d) Load Aggregator Agent (LAA) data

(a)

\begin{tabular}{|c|c|c|c|c|c|}
\hline \multicolumn{6}{|c|}{ LMA Data } \\
\hline Zone & DG & Load & $\begin{array}{c}\text { Total System } \\
\text { Load }\end{array}$ & DG Bid & Load Bid \\
\hline & & & & & \\
\hline & & & & & \\
\hline
\end{tabular}

(b)

\begin{tabular}{|l|l|}
\hline \multicolumn{2}{|c|}{ ZA Data } \\
\hline DG Information & Load Information \\
\hline & \\
\hline
\end{tabular}

(c)

\begin{tabular}{|c|c|c|c|}
\hline \multicolumn{4}{|c|}{ DGA Data } \\
\hline DG Type & Capacity & Availability & DG Bid \\
\hline Solar & $10 \mathrm{KW}$ & N/A & \\
\hline & & & \\
\hline
\end{tabular}

(d)

\begin{tabular}{|c|c|c|c|c|}
\hline \multicolumn{5}{|c|}{ LAA Data } \\
\hline $\begin{array}{c}\text { Load } \\
\text { Type }\end{array}$ & Usage Rate & Availability & $\begin{array}{c}\text { MW } \\
\text { Requirement }\end{array}$ & Load Bid \\
\hline Hybrid & $.10 \mathrm{MW} /$ Hour & $0500-2100$ & .15 & \\
\hline & & & & \\
\hline
\end{tabular}

\subsubsection{Load Management Strategy I}

Figure 4-15 represents sequence of actions performed by MAS in order to achieve the peak load reduction. 


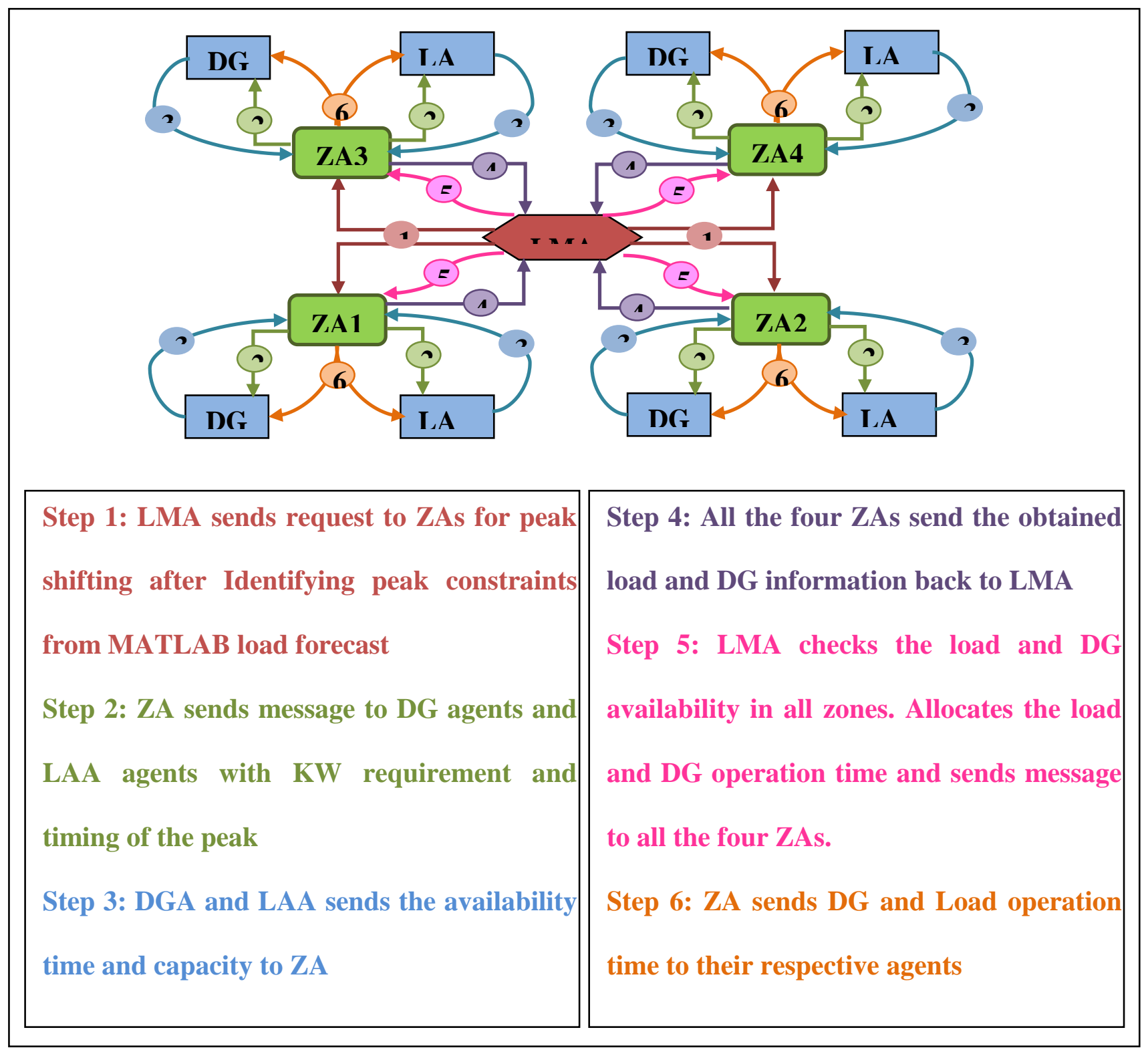

Figure 4- 15: MAS action sequences for peak load reduction by 15 percent in strategy I 
The LMA is programmed by considering following key points:

- Threshold is decided by deducting $15 \%$ from maximum peak value. Once the threshold has been decided, all the values above threshold are considered to be peaks

- Number of peaks to be shifted or supplied and peak area will be calculated

- All zonal load and DG data is aggregated to calculate the available times for MW shift

- Usage of the controllable load is assigned as blocks as depicted below

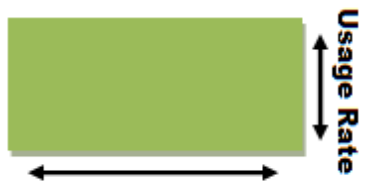

Usage Time

Figure 4- 16: Load usage blocks

- Loads which are available during peak are considered as a priority for load shifting. If there are no loads which are available for shifting their usage, DGs are assigned to supply the rest

- Conveying decided allocation to all zones

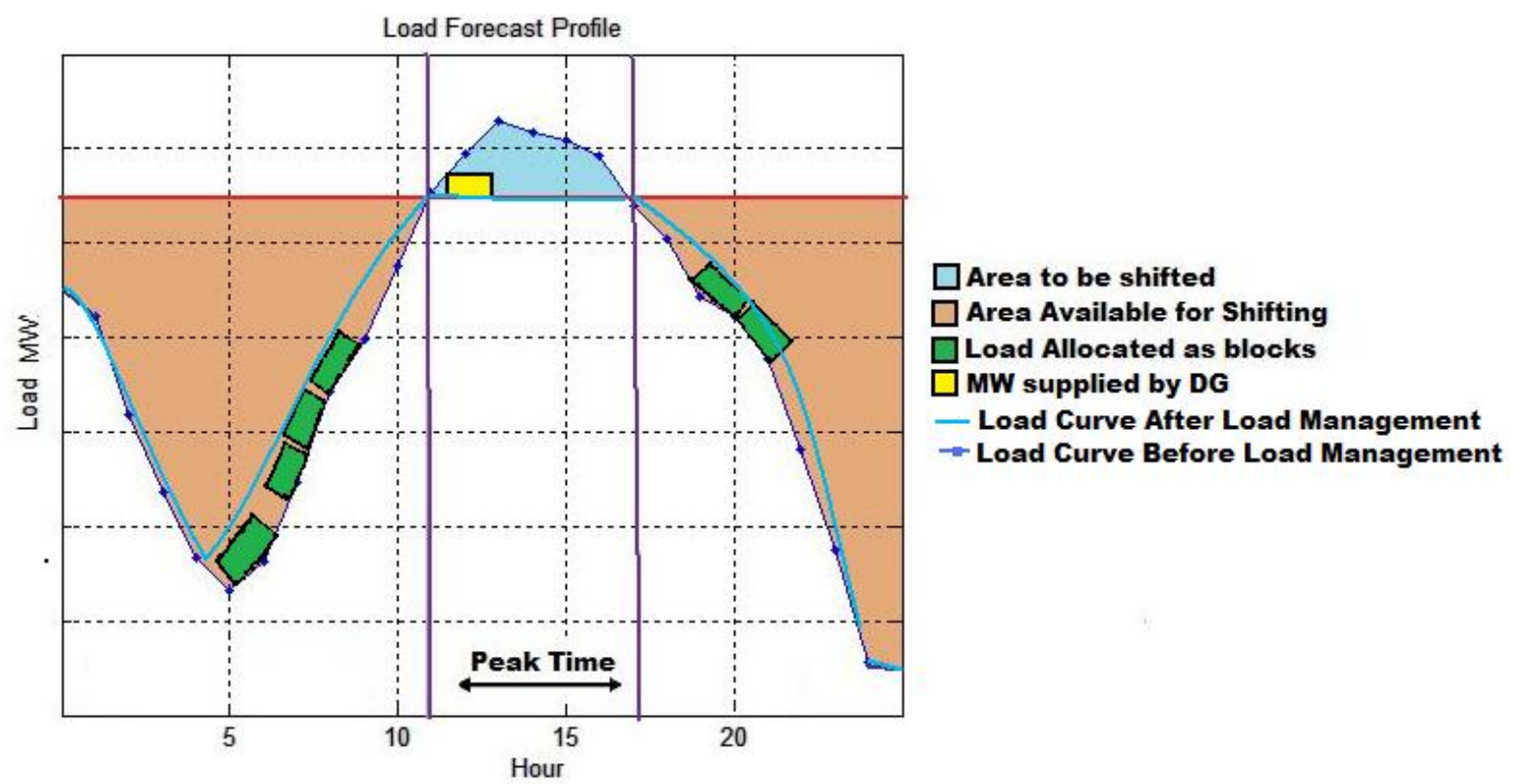

Figure 4- 17: Load profile with possible MW availability 
Figure 4-17 illustrates load profile representing peak time, peak MW and available MW for shifting.

\subsubsection{Load Management Strategy II}

Strategy II which is also been implemented using MAS is another attempt to reduce the peak by 15 percent but considering the price signal. Once the LMA identifies peak characteristics and sends the information to all the four ZAs, they in-turn send a signal to DGAs and LAA requesting their capacity and bid. The objective of the LMA will be to minimize the price to reduce the peak. The objective can be modeled as an optimization problem subject to equality and in-equality constraints. $\boldsymbol{J}$ as described below is a function of MW to be supplied by DG/ to be reduced by load and their bids. In-equality constraints are the minimum and maximum MW limit of the DGs and/or loads. The equality constraint is energy balance equation that is at the given instance the reduction of load and incremental of DG supply must satisfy the peak that needs to be reduced.

Minimize $J$

$J=\sum_{i=1}^{4} a_{i} * X_{i}+\sum_{j=1}^{4} b_{j} * Y_{j}$

Subject to

$\sum_{i=1}^{4} X_{i}+\sum_{j=1}^{4} Y_{j}=\mathrm{A}$

Bounds

$l_{i} \leq X_{i} \leq U_{i} ; i=0,1,2,4$

$m_{j} \leq Y_{j} \leq N_{j} ; j=0,1,2,4$

Where

$\boldsymbol{X}_{\boldsymbol{i}}$ - supply MW of $i_{t h}$ DG

$\boldsymbol{Y}_{\boldsymbol{i}}$ - reduced MW of $j_{t h}$ Load

$\boldsymbol{a}_{\boldsymbol{i}}$ - Bid of $i_{t h}$ DG to supply load (\$/MWh) 
$\boldsymbol{b}_{\boldsymbol{j}}$ - Bid of $j_{t h}$ Load to reduce load (\$/MWh)

A - Peak demand MW that needs to be reduced (MW)

$\boldsymbol{l}_{\boldsymbol{i}} \& \boldsymbol{U}_{\boldsymbol{i}^{-}}$Lower and Upper Limit of $i_{t h}$ DG respectively

$\boldsymbol{m}_{\boldsymbol{j}} \& \boldsymbol{N}_{\boldsymbol{i}^{-}}$Lower and Upper Limit of $j_{t h}$ Load respectively

IBM ILOG CPLEX Optimization studio has been interfaced with JADE to solve the optimization function with the DG and load constraints. Linear programming solver is used.

Figure 4-18 explains the LMA action sequence in the peak management strategy II. 


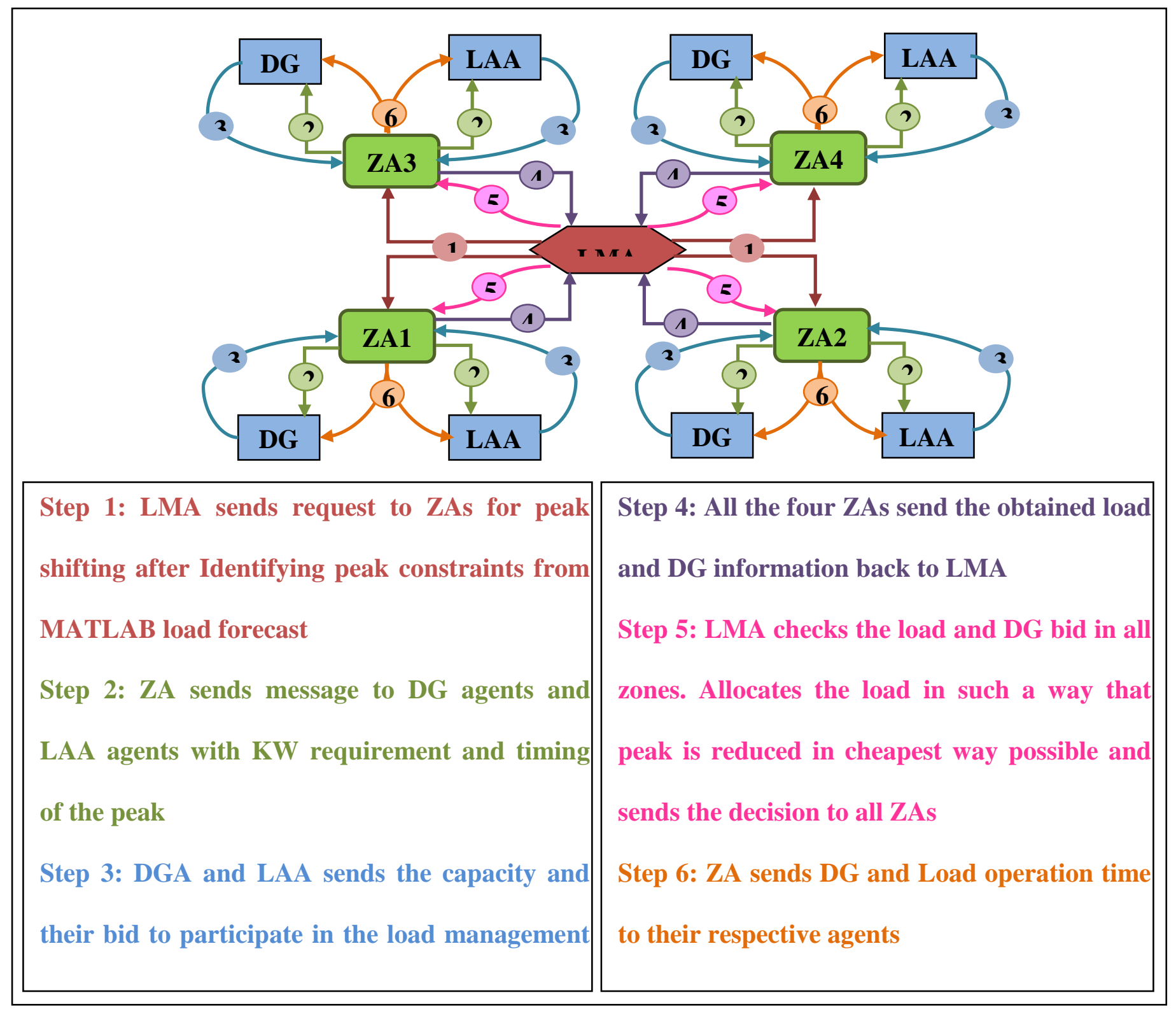

Figure 4- 18: MAS action sequences for peak load reduction by 15 percent in strategy II 


\subsection{Test Case Peak Reduction}

The load forecasted using developed load forecaster with values as in Table 4-1 are given as an input MAS. Figure 4-19 shows the output on JADE console

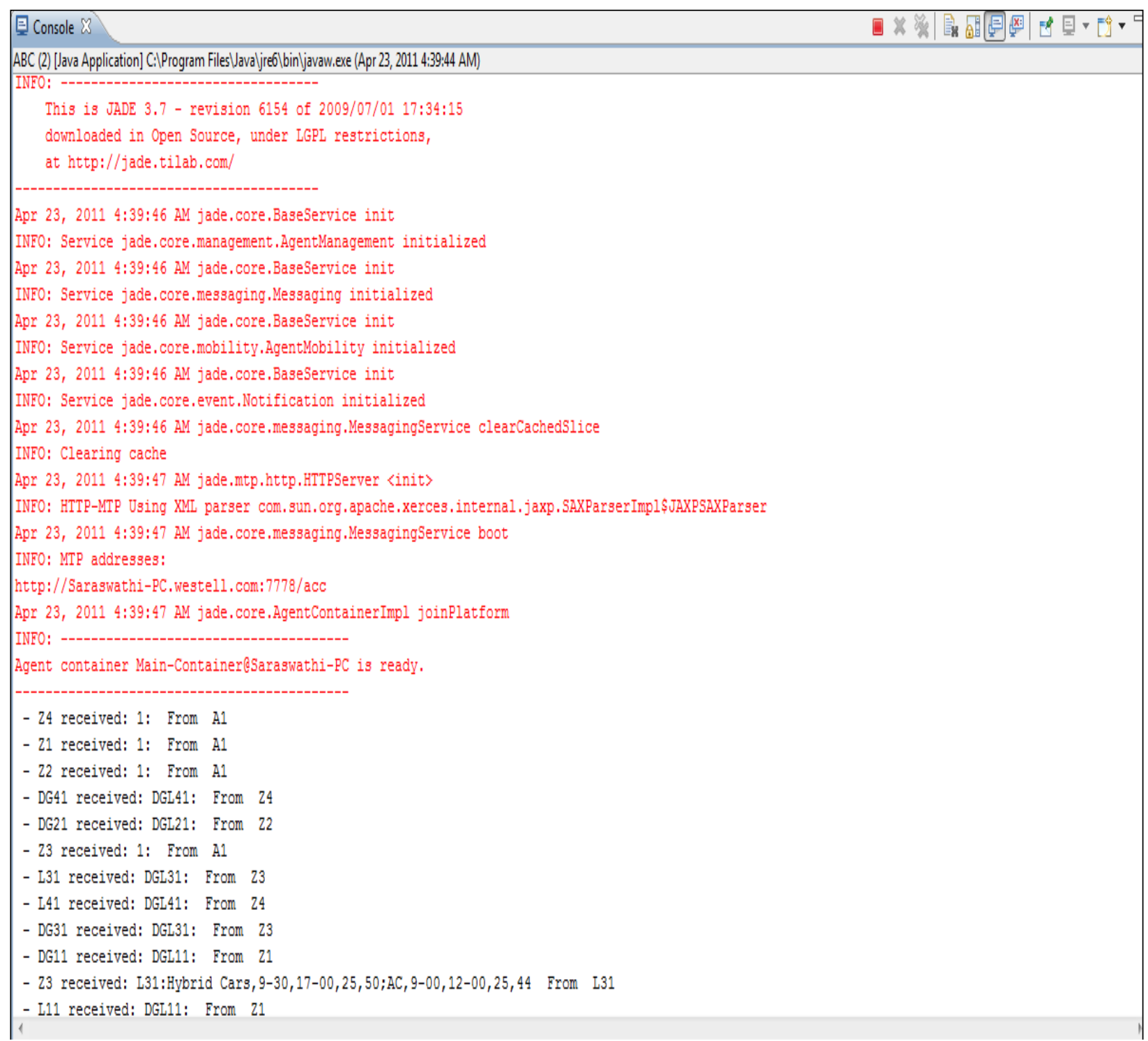

Figure 4- 19: MAS output from JADE console

Following is the output of MAS communicating to reduce the peak demand by 15 percent and the interpretation of the communication:

A1 is Load Management Agent (LMA), 
$\mathrm{Z1}, \mathrm{Z} 2, \mathrm{Z3}$, and Z4 are Zone Agents (ZA) in zone 1, 2, 3 and 4 respectively,

DG11, DG21, DG31, and DG41 are Distributed Generation Agents (DGA) in zone 1, 2, 3, and 4, L11, L21, L31, and L41 are Load Aggregator Agent (LAA) in zone 1, 2, 3, and 4.

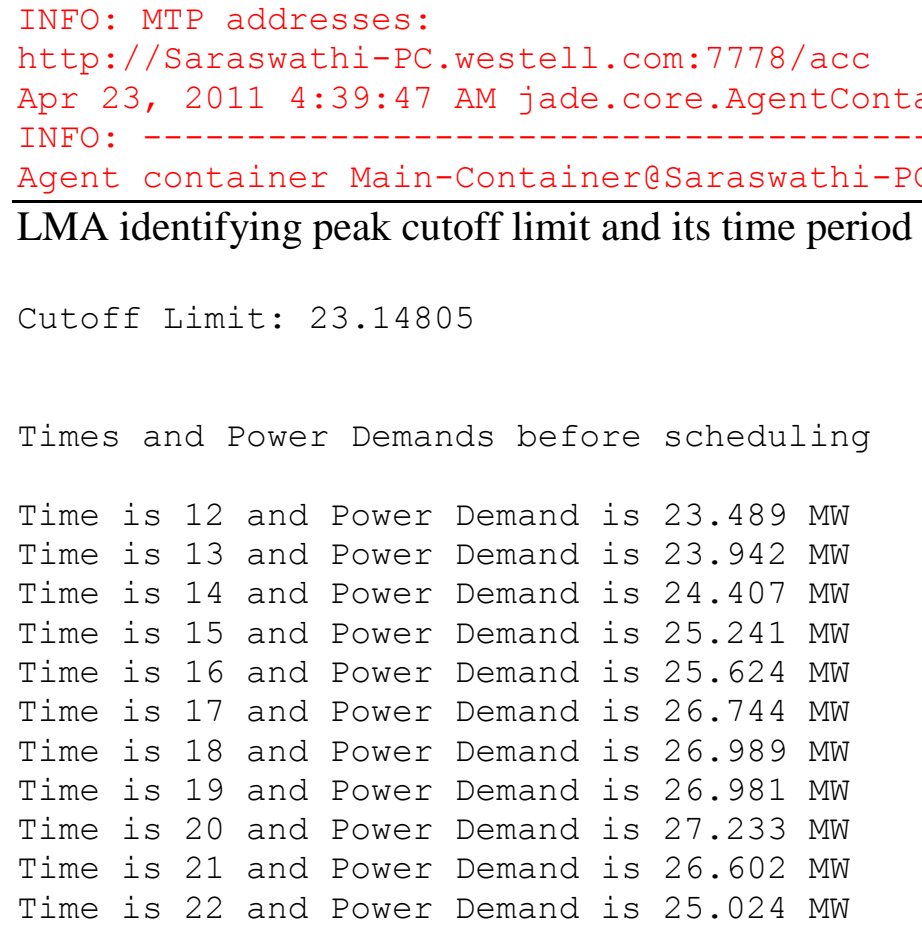

ZAs receiving request from LMA about DG and Load Availability

- Z4 received: 1: From A1

- Z1 received: 1: From A1

- Z2 received: 1: From A1

- Z3 received: 1: From A1

DGAs receiving request from ZA about DG Information

- DG41 received: DGL41: From Z4

- DG21 received: DGL21: From Z2

- DG31 received: DGL31: From Z3

- DG11 received: DGL11: From Z1

LAAs receiving request from $\mathrm{ZA}$ about $\mathrm{DG}$ Information

- L31 received: DGL31: From Z3

- L41 received: DGL41: From Z4

- L11 received: DGL11: From Z1

- L21 received: DGL21: From Z2

ZAs receiving DG Information from DGA

- Z1 received: DG11:Solar,0-00,23-00,4;Wind,0-00,23-00,12 From DG11

- Z2 received: DG21:Solar,4-00,6-00,1;Wind,12-00,16-00,9 From DG21

- Z3 received: DG31:Solar,4-00,6-00,3;Wind, 12-00,16-00,20 From DG31

- Z4 received: DG41:Solar,4-00,6-00,2;Wind,12-00,16-00,5 From DG41 
ZAs receiving Load Information from LAA

- Z1 received: L11:Hybrid Cars, 0-00,5-00, 25,1;AC, 9-00,12-00, 25, 2 From L11

- Z2 received: L21:Water Boilers, 4-00,17-00,1,2;AC,9-00,12-00,0.5,1;AC1,9-

$00,12-00,1,2$ From L21

- Z3 received: L31:Hybrid Cars, 9-30,17-00,1,1;CHP,9-00,12-00,0.5, 1 From L31

- Z4 received: L41:Hybrid Cars, 9-30,17-00,1,1;AC,9-00,12-00,1,2 From L41

\section{LMA receiving Load and DGs Information from ZAs}

- A1 received: DG11:Solar,0-00,23-00,4;Wind,0-00,23-00,12 From Z1

- A1 received: L41:Hybrid Cars,9-30,17-00,1,1;AC,9-00,12-00,1,2 From Z4

- A1 received: L21:Water Boilers, 4-00,17-00,1,2;AC,9-00,12-00,0.5,1;AC1,9-

$00,12-00,1,2$ From Z2

- A1 received: L31:Hybrid Cars, 9-30,17-00,1,1;CHP,9-00,12-00,0.5,1 From Z3

- A1 received: DG21:Solar,4-00,6-00,1;Wind,12-00,16-00,9 From Z2

- A1 received: DG31:Solar,4-00,6-00,3;Wind,12-00,16-00,20 From Z3

- A1 received: L11:Hybrid Cars, 0-00,5-00,25,1;AC,9-00,12-00,25,2 From Z1

\section{Peak Demand Reduction Conclusion}

Peak1: The target peak load reduction of 15 percent has been achieved for peak0 occurring between 12 and 22

\section{ZAs receiving reallocated schedules from LMA}

- Z1 received: DG11D:16,17,3.035949999999998:17,18,3.718449999999999:

$18,19,3.83695: 19,20,3.9589499999999997: 20,21,3.769449999999999: 21,22,2.664949$ $9999999993: 22,23,0.6373032239224921$ From A1

- Z2 received: L21D:4,5,1.0:5,6,0.26984999999999637 From A1

- Z3 received: L31D:9,10,0.5674499999999991 From A1

- Z4 received: L41D:9,10,0.06270059466019387 From A1

\section{DGs receiving reallocated schedules from ZAs}

- DG11 received: DG11D::16,17,3.035949999999998:17,18,3.718449999999999:

$18,19,3.83695: 19,20,3.9589499999999997: 20,21,3.769449999999999: 21,22,2.664949$ 9999999993:22,23,0.6373032239224921 From Z1

- L21 received: L21D::4,5,1.0:5,6,0.26984999999999637 From Z2

- L31 received: L31D::9,10,0.5674499999999991 From Z3

- L41 received: L41D::9,10,0.9028494053398082 From Z4

- DG41 received: DG41D::15,16,1.5542999999999942 From Z4

\section{MW after peak shifting}

WATTAGES AFTER SHIFTING PEAK LOAD AT EACH TIME PERIOD

Between 0 and 1: 18.801000000000002

Between 1 and 2: 17.269

Between 2 and 3: 16.167

Between 3 and 4: 15.635

Between 4 and 5: 17.494650000000004

Between 5 and 6: 17.140849999999997

Between 6 and 7: 18.6545 


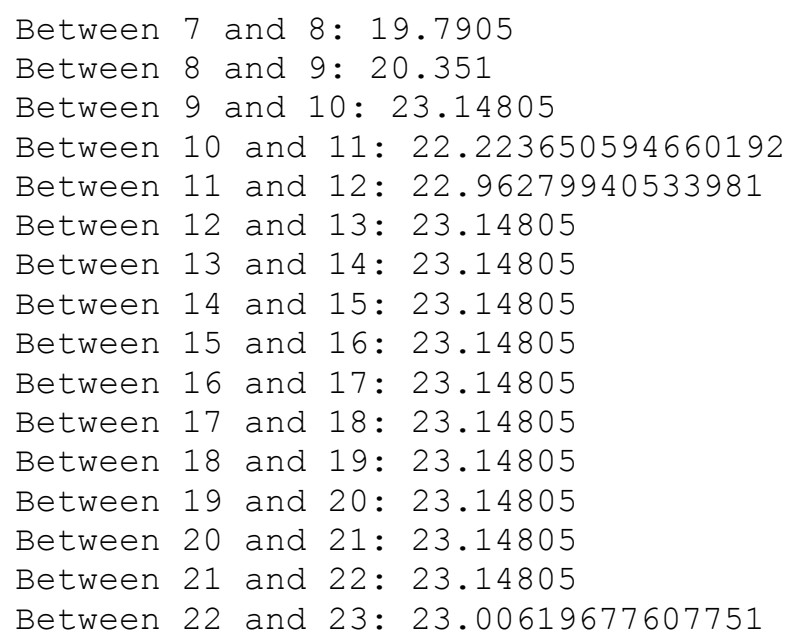

Figure 4-20 depicts actual system load, forecasted system load (system load before peak management) and system load after peak management.

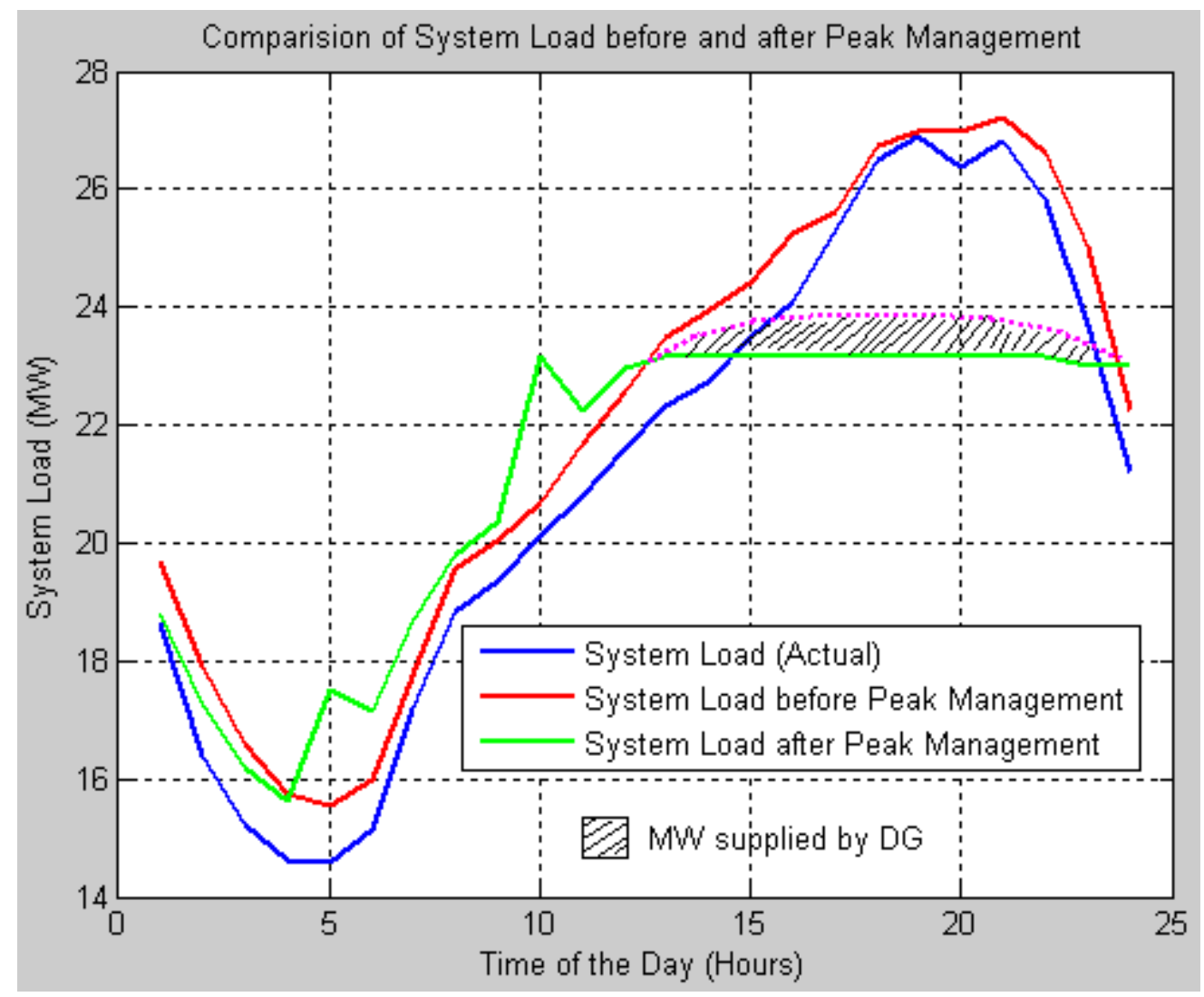

Figure 4- 20: Actual, forecasted and peak managed system load curves 


\subsection{Conclusions}

This chapter discusses the approach and its results in implementing load management system for smart distribution system. Load forecasting module is developed using neural networks toolbox and statistic toolbox provided by MATLAB. MAS is implemented using JADE for peak demand reduction using 2 strategies. Test case is executed to see the load forecast and peak demand reduction. 15 percent of peak demand reduction has been achieved on the forecasted data. 


\section{Chapter 5}

\section{CONCLUSIONS AND FUTURE WORK}

With the impact of industrialization and advancement in the way the electricity is being consumed, considerable change is seen in the way the power grid in planned, constructed and operated. To meet the ascending electricity needs and to make the grid more reliable various technologies are being adopted by the power grid. To save energy, reduce the electricity cost and increase the reliability it has been envisioned to overlay the present power grid with communicating capability and make it a smartgrid. Multi-Agent System (MAS) is one of those technologies which embeds intelligence into the grid enabling it be operated automatically.

\subsection{Conclusions}

In this present thesis work an attempt has been made to implant MAS into the power distribution system as a load management system using controllable loads and distributed generation resources. A network of agents at various level of distribution system i.e. at substation, zones, loads, and at distributed generation level forms a MAS for the power distribution system. The MAS takes the hourly forecasted data and estimates the power demand occurring time and keeps a target to reduce the peak by 15 percent. With the target to keep power demand reduced at 15 percent, the agents negotiate to check the availability of distributed generation units and controllable loads to manage the demand accordingly. 
The computational software package, MATLAB, is used to forecast the power demand. Using one year load data, dry bulb temperature, and the type of the day information, artificial neural networks tool box from MATLAB forecasts the electricity load. The load forecaster takes desired day's temperature forecast, type of the day weather it is a holiday or working day and forecast the hourly load for that day.

MAS are programmed using JADE. The load management system is assumed to be managing the service area categorized into four zones. Each zone is assumed to have a distributed generation and a load aggregator which has the information of the loads charging time, rate, and availability. The load management agent (LMA) is located at substation level, zone agent (ZA) at each zone, load aggregator (LA) at each aggregator, and DG agent (DGA) at each DG. All of the agents will have their respective device information. The load management is performed using two strategies.

Load Management Agent (LMA) receives hourly forecast of the power demand and identifies the peak demand occurring time and peak demand KW. It calculates the 15 percent of it and subtracts it from peak demand KW, keeping it as cutoff limit, LMA identifies time zone in which the demand is exceeding the cutoff limit. The total area above the cutoff limit line of the demand curve is intended to sift before and after the above identified time zone. The LMA send request to the zone agent to send the information about when and how much of the load is available for shifting. All the ZAs sends message to LA and DG agents of respective zone for required demand KW and demand availability information. DGA will reply back with DG capacity, availability, and their bid. LA will reply with controllable load availability, requirements and bid. 
ZA at four zone levels receives DGA's and LA's information and sends it back to LMA. Now two different strategies are executed to reduce the peak. In the first strategy, LMA will scrutinize the availability of loads and DERs with respect to time and schedules the turn-on and off time at each zone. In the second strategy LMA tries to minimize the cost to reduce the peak by solving the optimization problem based on the bids sent, thereby reducing the peak demand by 15 percent.

\subsection{Future Work}

Though Demand Management has been an area of interest to the power industry since a very long time, lately it is taking considerable changes because of penetration of communicating technology into the grid. And hence, the technology is still in the research arena with many possible extensions. Possible scopes for the extension of present thesis work are stated below,

- To make the system more reliable it is more than a necessity for the load management system to be decentralized. Though decentralization is achievable using MAS, a centralized load management agent manages the whole communication in this present work. So a decentralized model can be developed.

- Load forecaster deployed in the MATLAB has the hourly forecast data. For more accuracy 5-min/15-min forecast can be determined so as to achieve the peak demand reduction efficiently. For that 5-min/15-min historical load data must be used to calibrate the forecaster.

- In this work, demand is tried to be dispatched to match the generation. To make the problem more realistic, intermittent generation can be introduced by forecasting its generation and dispatch load accordingly. 
- To study how load is responding to the communication signals, load characteristics can be simulated using some simulation software so that the simulation responds to MAS showing load's turn on and turn off characteristics.

- Load forecaster is developed by considering past one year load data. Including past four years load data will minimize the forecasted load error.

- In the MAS model, only one load aggregator agent in each zone is considered. To make it more practically implementable, number of load aggregator agents in each zone can be increased.

- The bidding scenario can be made more realistic by introducing different bidding prices according to the time of the day. 


\section{REFERENCES}

[1] http://www.pickocc.org/publications/electric/Understanding_Distributed_Generation.pdf

[2] David Andrews, “National Grid's use of Emergency Diesel Standby Generator's in Dealing with Grid Intermittency and Variability Potential Contribution in Assisting Renewables", Open university conference on Intermittence, $24^{\text {th }}$ January, 2006

[3] Yoav Shoham and Kevin Leyton-Brown. Multiagent Systems: Algorithimic, Game Theoretic and Logical Foundations. Shoham and Leyton-Brown, 2009, 2010

[4] Fabio Bellifemine, Giovanni Caire and Dominic Greenwood. Developing multi-agent systems with JADE. John Wiley \& Sons, Ltd, 2007

[5] Magid Nikraz, Giovanni Caire, and Parisa A. Bahri "A Methodology for the Analysis and Design of Multi-Agent Systems using JADE”, International Journal of Computer Systems Science \& Engineering, May 2006

[6] G. Weiss, "Multi-agent Systems: A Modern Approach to Distributed Artificial Intelligence," The MIT Press, 2000

[7] M. Wooldridge, N. R. Jennings, "Intelligent agents: Theory and Practice," The Knowledge Engineering Review, Vol. 10, Issue 2, pp 115 - 152, 1995

[8] S. Russell and P.Norvig. Artificial Intelligence: A modern approach. Printice-Hall, 1995

[9] Scott A. Moore, "KQML \& FLBC: Contrasting Agent Communication Languages", Proceedings of the 32nd Hawaii International Conference on System Sciences - 1999

[10] Tim Finin, Richard Fritzson, Don McKay and Robin McEntire, "KQML as an Agent Communication Language", The Proceedings of the Third International Conference on Information and Knowledge Management (CIKM'94), ACM Press, November 1994

[11] Robert Neches, Richard Fikes, Tim Finin, Thomas Gruber, Ramesh Patil, Ted Senator, and 
William R. Swartout, "Enabling Technology for Knowledge Sharing", AI Magazine Volume 12 Number 3, 1991

[12] The Foundation for Physical Intelligent Agents, http://www.fipa.org

[13] FIPA 97 specifications, version 2.0, ACL, http://www.fipa.org

[14] M. Bagic and M. Kunstic, "Reducing Dependency on Network Reliability and Availability by Encapsulating the Negotiation Functionalities in ACL Messages", 8th International Conference on Telecommunications - ConTEL 2005

[15] The Energy Independence and Security Act of 2007 www1.eere.energy.gov/femp/pdfs/eisa_femp.pdf

[16] Bob Saint, "Rural Distribution System Planning using Smart Grid Technologies", IEEE Rural Electric Power Conference, 2009

[17] Richard E. Brown, "Impact of Smart Grid on Distribution System Design”, IEEE Power and Energy Society General Meeting, 2008

[18] EPRI's IntelliGrid ${ }^{\mathrm{SM}}$, http://intelligrid.epri.com/

[19] Mark McGranaghan and Frank R. Goodman, Jr., "EPRI Research Plan for Advanced Distribution Automation”, IEEE Power Engineering Society General Meeting, 2005

[20] The NETL Smart Grid Implementation Strategy, http://www.netl.doe.gov/smartgrid/

[21] GridWise Alliance, http://www.gridwise.org/

[22] GridWise Alliance Missions and Goals, 2010

[23] XUE Ligong, ZHOU Zude and LIU Quan, “Application of Multi-agent in Intelligent Manufacturing System", International Conference on Computer Science and Software Engineering, 2008

[24] Hayfa ZGAYA and Slim HAMMADI, "Assignment and Integration of Distributed 
Transport Services in Agent-Based Architecture", Proceedings of the IEEE/WIC/ACM International Conference on Intelligent Agent Technology, 2006

[25] Zhenjiang Li, Feng He, Qingming Yao and Fei-Yue Wang, "Signal Controller Design for Agent-Based Traffic Control System", Proceedings of the IEEE International Conference on Networking, Sensing and Control, 2007

[26] Li Liu, Kevin P. Logan, David A. Cartes and Sanjeev K. Srivastava, "Fault Detection, Diagnostics, and Prognostics: Software Agent Solutions", IEEE transactions on vehicular technology, VOL. 56, NO. 4, 2007

[27] Gaofeng Xiong, Shigeru Okuma and Hideki Fujita, "Multi-agent Based Experiments on Uniform Price and Pay-as-Bid Electricity Auction Markets", IEEE International Conference on Electric Utility Deregulation, Restructuring and Power Technologies, 2004

[28] Rui Duan and Geert Deconinck, "Future Electricity Market Interoperability of a Multi-agent Model of the Smart Grid", International Conference on Networking, Sensing and Control, 2010

[29] Steve Bou Ghosn, Prakash Ranganathan, Saeed Salem, Jingpeng Tang, Davin Loegering and Kendall E. Nygard, "Agent-oriented Designs for a Self Healing Smart Grid”, 1st IEEE International Conference on Smart Grid Communications, 2010

[30] Qingle Pang, Houlei Gao and Xiang Minjiang, "Multi-agent based Fault Location Algorithm for Smart Distribution Grid", 10th IET International Conference on Developments in Power System Protection, 2010

[31] David A. Cohen, "GridAgentsTM: Intelligent Agent Applications for Integration of Distributed Energy Resources within Distribution Systems", Power and Energy Society General Meeting, 2010 
[32] Frances Brazier, Frank Cornelissen, Rune Gustavsson, Catholijn M. Jonker, Olle Lindeberg, Bianca Polak and Jan Treur, "Agents Negotiating for Load Balancing of Electricity Use", Proceedings of the International Conference on Distributed Computing Systems, 1998

[33] Zita A. Vale, Carlos Ramos Hugo Morais, Pedro Faria and Marco Silva, "The Role of Demand Response in Future Power Systems", Transmission \& Distribution Conference \& Exposition: Asia and Pacific, 2009

[34] C.J. Warmer, M.P.F. Hommelberg, B. Roossien, J.K. Kok and J.W. Turkstra, “A field test using agents for coordination of residential micro-chp", International Conference on Intelligent Systems Applications to Power Systems, 2007

[35] Prakash R. Thimmapuram, Jinho Kim, Audun Botterud, and Youngwoo Nam, "Modeling and Simulation of Price Elasticity of Demand Using an Agent-Based Model", Innovative Smart Grid Technologies, 2010

[36] K. Morgan, H. Mullany, and M. Walsh, "Implementation of a Novel Peak Demand Reduction Scheme", Power Systems Conference and Exposition, 2004

[37] Richard E. Crane, Harold G. Lorsch, Richard L. Oswald, "Thermal Storage of Off--Peak Electrical Energy in Solar Heating and Cooling Systems", IEEE Conference on Decision and Control including the 17th Symposium on Adaptive Processes, 1978

[38] John Byme, Young Doo Wang, Ralph Nigro and William Bottenberg, "Commercial Building Demand-Side Management Tools: Requirement for Dispatchable Photovoltaic Systems", Conference Record of the Twenty Third IEEE Photovoltaic Specialists Conference, 1993

[39] A. J. Hoffman, "Peak demand control in commercial buildings with target peak adjustment based on load forecasting", Proceedings of the 1998 IEEE International Conference on 
Control Applications, 1998

[40] Ali Feliachi, John E. Saymansky, Muhammad A. Choudhry and J. Ed Sneckenberger, “Are All Smart Grids Equal?”, 6th International Conference on Electrical Engineering, 2010

[41] MathWorks, http://www.mathworks.com/products/matlab/

[42] Java Agent Development Framework, http://jade.tilab.com/

[43] Fabio Bellifemine, Giovanni Caire, Tiziana Trucco and Giovanni Rimassa, "JADE Programmer's Guide"

[44] The MathWorks Database Toolbox, http://www.mathworks.com/products/database

[45] IBM ILOG CPLEX Optimization

01.ibm.com/software/integration/optimization/cplex-optimizer/ 